\author{
Universidade de São Paulo \\ Instituto de Física
}

\title{
Gravidade de Lovelock e a correspondência AdS/CFT
}

\author{
Anderson Seigo Misobuchi
}

Orientador: Prof. Dr. Diego Trancanelli

Dissertação apresentada ao Instituto de Física da Universidade de São Paulo como requisito para o título de Mestre em Ciências

Comissão examinadora:

Prof. Dr. Diego Trancanelli (USP)

Prof. Dr. Betti Hartmann (USP)

Prof. Dr. Horatiu Nastase (UNESP)

São Paulo

2016 
FICHA CATALOGRÁFICA

Preparada pelo Serviço de Biblioteca e Informação do Instituto de Física da Universidade de São Paulo

Misobuchi, Anderson Seigo

Gravidade de Lovelock e a correspondência AdS/CFT. São Paulo, 2016.

Dissertação (Mestrado) - Universidade de São Paulo. Instituto de Física. Depto. de Física Matemática.

Orientador: Prof. Dr. Diego Trancanelli

Área de Concentração: Teoria de Cordas.

Unitermos: 1. Física de alta energia; 2. Física teórica;

3. Teoria de gauge.

USP/IF/SBI-019/2016 


\author{
University of São Paulo \\ Physics Institute
}

\title{
Lovelock gravity and the $\mathrm{AdS} / \mathrm{CFT}$ correspondence
}

\author{
Anderson Seigo Misobuchi
}

Advisor: Prof. Dr. Diego Trancanelli

Dissertation presented to the Physics Institute of University of São Paulo as a requirement to the title of Master of Science

Examining committee:

Prof. Dr. Diego Trancanelli (USP)

Prof. Dr. Betti Hartmann (USP)

Prof. Dr. Horatiu Nastase (UNESP)

São Paulo

2016 



\section{Acknowledgements}

First of all, I would like to express my sincere gratitude to my advisor Prof. Diego Trancanelli for all he have taught me. I am indebted to him for stimulating my interest in theoretical physics and for his guidance that helped me in all the time of research and writing of this thesis.

I thank Prof. Gustavo Burdman, with whom I have learned the principles of quantum field theory.

I thank all my colleagues at the Physics Institute who made it such a delightful place to work. In particular, I am grateful to Viktor Jahnke for the fruitful discussions during our collaboration. A special thanks goes to Renato Critelli, whom I have known for such a long time and who have been a great friend to me.

I thank FAPESP for the financial support under grant 2014/07840-7.

I thank Thainã for her nice advices.

Special thanks to Bia, the person who is always there to eat japanese food with me.

Finally, I want to thank my parents Maria and Carlos, and my sister Katia, whose unconditional love and support have made everything possible. 


\section{Resumo}

Misobuchi, A.S. Gravidade de Lovelock e a correspondência AdS/CFT. Dissertação de mestrado - Instituto de Física, Universidade de São Paulo, São Paulo, 2016.

A correspondência AdS/CFT é uma notável ferramenta no estudo de teorias de gauge fortemente acopladas que podem ser mapeadas em uma descrição gravitacional dual fracamente acoplada. A correspondência é melhor entendida no limite em que ambos $N$ e $\lambda$, o rank do grupo de gauge e o acoplamento de 't Hooft da teoria de gauge, respectivamente, são infinitos. Levar em consideração interações com termos de curvatura de ordem superior nos permite considerar correções de $\lambda$ finito. Por exemplo, a primeira correção de acoplamento finito para supergravidade tipo IIB surge como um termo de curvatura com forma esquemática $\alpha^{\prime 3} R^{4}$.

Neste trabalho investigamos correções de curvatura no contexto da gravidade de Lovelock, que é um cenário simples para investigar tais correções pois as suas equações de movimento ainda são de segunda ordem em derivadas. Esse cenário também é particularmente interessante do ponto de vista da correspondência AdS/CFT devido a sua grande classe de soluções de buracos negros assintoticamente AdS.

Consideramos um sistema de gravidade AdS-axion-dilaton em cinco dimensões com um termo de Gauss-Bonnet e encontramos uma solução das equações de movimento, o que corresponde a uma black brane exibindo uma anisotropia espacial, onde a fonte da anisotropia é um campo escalar linear em uma das coordenadas espaciais. Estudamos suas propriedades termodinâmicas e realizamos a renormalização holográfica usando o método de Hamilton-Jacobi. Finalmente, usamos a solução obtida como dual gravitacional de um plasma anisotrópico fortemente acoplado com duas cargas centrais independentes, $a \neq c$. Calculamos vários observáveis relevantes para o estudo do plasma, a saber, a viscosidade de cisalhamento sobre densidade de entropia, a força de arrasto, o parâmetro de jet quenching, o potencial entre um par quark-antiquark e a taxa de produção de fótons.

Palavras-chave: correspondência gauge-gravidade, holografia e o plasma de quark e gluons, gravidade de curvatura mais elevada. 


\section{Abstract}

Misobuchi, A.S. Lovelock Gravity and the AdS/CFT correspondence 2016. Master degree dissertation - Physics Institute, University of São Paulo, São Paulo, 2016.

The AdS/CFT correspondence is a remarkable tool in the study of strongly coupled gauge theories which can be mapped to a dual, weakly coupled gravitational description. The correspondence is best understood in the limit in which both $N$ and $\lambda$, the rank of the gauge group and the 't Hooft coupling of the gauge theory, respectively, are infinite. Accounting for higher curvature interactions allows one to begin to consider finite $\lambda$. For example, the leading finite coupling corrections to type IIB supergravity arise as stringy corrections with schematic form $\alpha^{\prime 3} R^{4}$.

In this work we investigate higher curvature corrections in a simpler scenario, the Lovelock gravity. Lovelock gravity is a nice framework to investigate such corrections since its equations of motion are still second order in derivatives and is particularly interesting from the point of view of the AdS/CFT correspondence because a large class of asymptotically AdS black holes solutions are known.

We consider five-dimensional AdS-axion-dilaton gravity with a Gauss-Bonnet term and find a solution of the equations of motion which corresponds to a black brane exhibiting a spatial anisotropy, with the source of the anisotropy being an axion field linear in one of the spatial coordinates. We study its thermodynamics and we carry out the holographic renormalization using the Hamilton-Jacobi approach. Finally, we use the solution as a gravity dual to a strongly coupled anisotropic plasma with two independent central charges, $a \neq c$. We compute several observables relevant to the study of the plasma, namely, the shear viscosity over entropy density ratio, the drag force, the jet quenching parameter, the quarkonium potential and the thermal photon production.

Keywords: Gauge-gravity correspondence, Holography and quark-gluon plasmas, Higher curvature gravity. 


\section{Contents}

List of Figures vii

List of Tables $\quad$ ix

1 Overview 1

2 AdS/CFT correspondence $\quad 4$

2.1 Arguments for plausibility . . . . . . . . . . . . . . . 5

2.2 Basics of string theory . . . . . . . . . . . . . . . 9

2.2 .1 Generalities . . . . . . . . . . . . . . . . 9

2.2 .2 Superstring . . . . . . . . . . . . . . . . 10

2.2 .3 Type IIB supergravity . . . . . . . . . . . . . . . . . . 13

2.3 D-branes: the two pictures . . . . . . . . . . . . . . . . 14

2.3.1 Open string picture . . . . . . . . . . . . . . 14

2.3.2 Closed string picture . . . . . . . . . . . . . . . 16

2.4 Statement of the AdS/CFT correspondence . . . . . . . . . . . . 18

2.5 Correlation functions . . . . . . . . . . . . . . . . . . . . 19

3 Lovelock gravity $\quad 21$

3.1 Non-coordinate basis . . . . . . . . . . . . . . . . . . . 22

3.2 Lovelock action . . . . . . . . . . . . . . . . . . . . 22

3.3 Equations of motion and vacuum solutions . . . . . . . . . . . . . . 24

3.4 Black hole solutions . . . . . . . . . . . . . . . . . 25

3.5 Gauss-Bonnet black holes . . . . . . . . . . . . . . . . 26

3.6 Lovelock and AdS/CFT correspondence . . . . . . . . . . . . . . 27

3.6.1 Violation of the KSS bound . . . . . . . . . . . . . . . 28

3.6.2 Unitarity of the dual CFT . . . . . . . . . . . . . . . . 28

3.6.3 Positivity of the energy flux . . . . . . . . . . . . . . . 30

3.6.4 Causality violation . . . . . . . . . . . . . . . . . 31 
4 Chern-Simons diffusion rate from higher curvature gravity

4.1 Chern-Simons diffusion rate . . . . . . . . . . . . . . . . 34

4.2 Gravity setup and results . . . . . . . . . . . . . . 36

4.3 Discussion . . . . . . . . . . . . . . . . . . . 39

5 Anisotropic black branes in higher curvature gravity 41

5.1 Action and solution . . . . . . . . . . . . . . . . . 42

5.2 Holographic renormalization . . . . . . . . . . . . . . . . 45

5.2 .1 Radial evolution Hamiltonian . . . . . . . . . . . . . . . . . 46

5.2 .2 Recursive method . . . . . . . . . . . . . . . 48

5.2 .3 Fefferman-Graham expansions . . . . . . . . . . . . . . 53

5.2 .4 The 1-point functions . . . . . . . . . . . . . . . 54

5.2 .5 Central charges ...................... 56

5.3 Boundary stress tensor . . . . . . . . . . . . . . . . . . 57

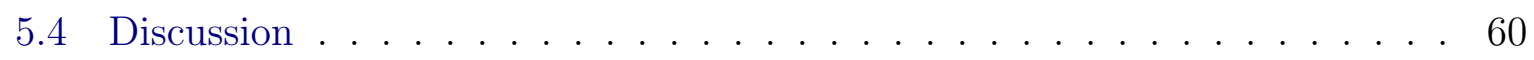

6 Probing strongly coupled anisotropic plasmas from higher curvature $\begin{array}{ll}\text { gravity } & 61\end{array}$

6.1 Gravity setup . . . . . . . . . . . . . . . . 62

6.2 Shear viscosity . . . . . . . . . . . . . . . . 63

6.3 Drag force . . . . . . . . . . . . . . . . . . 65

6.4 Jet quenching parameter . . . . . . . . . . . . . . . . . 68

6.5 Quarkonium static potential ..................... 71

6.6 Photon production . . . . . . . . . . . . . . . . 74

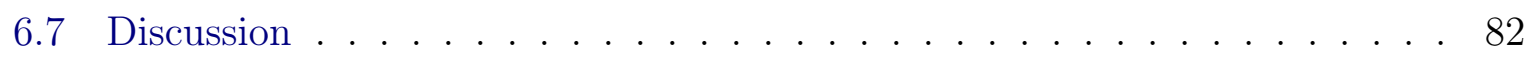

7 Conclusion $\quad 84$

$\begin{array}{ll}\text { A Symmetries in quantum field theories } & 87\end{array}$

B Derivation of the anisotropic gravity solution $\quad 90$

$\begin{array}{ll}\text { C Shear viscosity from Kubo formula } & 93\end{array}$

D Drag force for a general background and arbitrary direction $\quad 95$

$\begin{array}{lll}\text { E Jet quenching parameter for an arbitrary motion } & 98\end{array}$

F Quarkonium static potential in generic background $\quad 102$

$\begin{array}{ll}\text { Bibliography } & 104\end{array}$ 


\section{List of Figures}

2.1 Genus expansion for an amplitude with two closed strings going into two closed strings. . . . . . . . . . . . . . . . . . . 7

2.2 Left: the renormalization group flow from the QFT point of view. Right: the energy scale of the QFT identified as an extra dimension $z \ldots \ldots$. . .

2.3 The closed string picture: inside the throat, we have interacting closed strings in $A d S_{5} \times S^{5}$. Outside the throat, we have free strings in flat space. 17

3.1 The shock wave profile corresponds to the line $u=0$. The graviton correponds to the solid line $v=$ const. After colliding with the shock wave, the graviton may return in a position such that $\Delta v<0$, outside its own light-cone and thus violating causality. . . . . . . . . . . . . . . . 33

4.1 (Left) The factors $H^{(0)}\left(\lambda_{\mathrm{GB}}\right)$ (red, solid curve) and $H^{(1)}\left(\lambda_{\mathrm{GB}}\right)$ (blue, dashed curve) as functions of $\lambda_{\mathrm{GB}}$. (Right) The same factors as functions of $\eta / s$. The plots are exact in $\lambda_{\mathrm{GB}}$ and in $\eta / s$, whose allowed ranges are obtained from eqs. (4.13) and (4.20). In these ranges, the corrections to eq. (4.4) are finite and cannot make the diffusion rate arbitrarily small.

5.1 The metric functions at order $O\left(a^{2}\right)$ for $\lambda_{\mathrm{GB}}=0.2$ (left) and $\lambda_{\mathrm{GB}}=-0.2$ (right).

6.1 Drag force normalized by the isotropic result as a function of $\left(\lambda_{\mathrm{GB}}, \frac{a}{T}\right)$. Here we have fixed $v=0.3$. Left: Motion along the anisotropic direction. Right: Motion along the direction transversal to the anisotropy.

6.2 Drag force normalized by the isotropic result as a function of $\left(\lambda_{\mathrm{GB}}, v\right)$. Here we have fixed $\frac{a}{T}=0.2$. Left: Motion along the anisotropic direction. Right: Motion along the direction transversal to the anisotropy. For other values of $\frac{a}{T}$ the results were qualitatively the same. . . . . . . . . . . . . 68 
6.3 Left: Jet quenching parameter as a function of $(\theta, \varphi)$. We have set $\lambda_{\mathrm{GB}}=0.1$ and $a / T=0.33$. Right: The jet quenching parameter as a function of $\left(\lambda_{\mathrm{GB}}, \frac{a}{T}\right)$. We have set $\theta=\varphi=\pi / 4$. Both plots were normalized by the isotropic result $(6.32) \ldots \ldots \ldots \ldots \ldots \ldots$

6.4 Quark-antiquark potential $V_{Q \bar{Q}}$ as a function of their separation $L$ for different values of the Gauss-Bonnet coupling: $\lambda_{\mathrm{GB}}=-0.1$ (red, dotted), $\lambda_{\mathrm{GB}}=0$ (black, solid) and $\lambda_{\mathrm{GB}}=0.1$ (blue, dashed). For all curves $a / T \approx 0.3$ and $\theta=\pi / 4 \ldots \ldots \ldots \ldots \ldots \ldots \ldots \ldots$

6.5 (a) Screening length $L_{s}\left(\lambda_{\mathrm{GB}}, a\right)$ normalized with respect to the isotropic result $L_{\text {iso }}=L_{s}\left(\lambda_{\mathrm{GB}}=0, a=0\right)$ for $\theta=0$. (b) Ratio $L_{\perp} / L_{\|}$, where $L_{\perp}$ is the screening length calculated at $\theta=\pi / 2$, and $L_{\|}$is the screening length calculated at $\theta=0 \ldots \ldots \ldots \ldots \ldots \ldots$

6.6 Screening length $L_{s}$ as a function of $a / T$ for three different quarkonium orientations: $\theta=0$ (black, solid), $\theta=\pi / 4$ (purple, dashed) and $\theta=\pi / 2$ (blue, dotted). The Gauss-Bonnet coupling is fixed $\lambda_{\mathrm{GB}}=0 . \ldots$. . . . .

6.7 Momentum $\vec{k}$ and polarization vectors $\vec{\epsilon}_{(1)}$ and $\vec{\epsilon}_{(2)}$. The $S O(2)$ rotational symmetry in the $x y$-plane allows us to choose the momentum lying in the $x z$-plane, forming an angle $\vartheta$ with the $z$-direction. Both polarization vectors are orthogonal to $\vec{k}$. We chose $\vec{\epsilon}_{(1)}$ oriented along the $y$-direction and $\vec{\epsilon}_{(2)}$ contained in the $x z$-plane. . . . . . . . . . . . . . . . .

6.8 The trace of the spectral density $\chi_{\mu}^{\mu}\left(\lambda_{\mathrm{GB}}, a, \vartheta\right)$ normalized with respect to the isotropic result (6.59). All the spectral densities were calculated at the same temperature $T_{0}=0.316698$. The color of the curves identify the value of the $\lambda_{\mathrm{GB}}$ parameter as: red curves $\left(\lambda_{\mathrm{GB}}=-0.1\right)$, brown curves $\left(\lambda_{\mathrm{GB}}=-0.05\right)$, black curves $\left(\lambda_{\mathrm{GB}}=0\right)$, purple curves $\left(\lambda_{\mathrm{GB}}=0.05\right)$ and blue curves $\left(\lambda_{\mathrm{GB}}=0.1\right)$. In (a), the angle of emission is fixed $(\vartheta=0)$ and we have solid curves $(a=0.2)$, dashed curves $(a=0.1)$ and dotted curves $(a=0)$. In (b), the anisotropy is fixed $(a=0.2)$ and we have solid curves $(\vartheta=0)$, dot-dashed curves $(\vartheta=\pi / 4)$ and

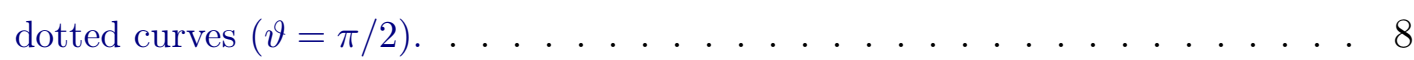

6.9 Total photon production rate as function of $\mathrm{w}=k^{0} / 2 \pi T_{0}$. From top to bottom, the value of the Gauss-Bonnet coupling is identified as $\lambda_{\mathrm{GB}}=0.1$ (blue), $\lambda_{\mathrm{GB}}=0.05$ (purple), $\lambda_{\mathrm{GB}}=0$ (black), $\lambda_{\mathrm{GB}}=-0.05$ (brown), $\lambda_{\mathrm{GB}}=$ -0.1 (red). We have fixed $\vartheta=0$ and $a=0.2$. The results for different angles are very similar to the plot above due to the smallness of the anisotropy. 


\section{List of Tables}

2.1 Massless closed string states. . . . . . . . . . . . . . . . . 13

2.2 Three levels of the AdS/CFT correspondence. . . . . . . . . . . . . . . 19

6.1 Summary of the effect of the Gauss-Bonnet coupling $\lambda_{\mathrm{GB}}$ on several observables. We also present the finite 't Hooft corrections of type $\alpha^{\prime 3} R^{4}$. The comparison is taken w.r.t. the respective $\mathcal{N}=4 \mathrm{SYM}$ result at same

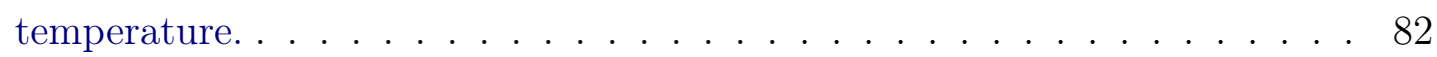




\section{Chapter 1}

\section{Overview}

The AdS/CFT correspondence [1-3] represents a remarkable tool in the study of strongly coupled gauge theories which can be mapped into a dual, weakly coupled gravitational description. For example, in ultra-relativistic heavy ion collision experiments, large nuclei are collided and a state of matter with very high temperature and density, the quark gluon plasma (QGP), is produced. Results obtained in experiments at the Relativistic Heavy Ion Collider (RHIC) [4,5] and at the Large Hadron Collider (LHC) [6] indicate that the QGP behaves as a strongly coupled system which cannot be studied by traditional perturbation theory methods.

The correspondence is best understood in the limit in which both $N$ and $\lambda$, the rank of the gauge group and the 't Hooft coupling of the gauge theory, respectively, are infinite. Investigating departures from this limit implies introducing $\alpha^{\prime}$ and loop corrections for the string and it is clearly of the utmost importance for a series of reasons, from achieving a deeper understanding of how the correspondence works in larger regions of the parameter space, to modeling more realistic gauge theory systems, where $N$ and $\lambda$ are obviously not infinite. Accounting for higher curvature interactions allows one to begin to consider finite $\lambda$ corrections. For example, the leading finite coupling corrections to type IIB supergravity arise as stringy corrections with schematic form $\alpha^{\prime 3} R^{4}[7]$.

One more modest approach is to consider simple generalizations of Einstein gravity, where higher curvature corrections are under control and calculable, in the hope to gain some qualitative understanding of the effects they might have and, perhaps, uncover some universal properties. A well-studied family of corrections is represented by Lovelock theories of gravity [8-11]. ${ }^{1}$ These theories are defined as natural extensions of the Einstein-Hilbert action to dimensions higher than four. The main characteristic of Lovelock gravities is the fact that, albeit being defined in terms of higher curvature/derivative

\footnotetext{
${ }^{1}$ Reviews on Lovelock theories with an emphasis on their relevance in the AdS/CFT context can be found in, e.g., [12-14].
} 
terms, they yield second order equations of motion and are free of pathologies. The first Lovelock correction, which is present already in five dimensions, is given by the GaussBonnet (GB) term, which is quadratic in the curvature. Another point of interest in this correction is that, besides being calculable, it possesses a wealth of exact black hole solutions with AdS asymptotics; see e.g. [15-17] for a comprehensive review.

It is clearly worthwhile to try to find as many new solutions as possible and increase the arena of models where explicit computations can be performed. With this motivation in mind, in this work we consider a GB correction to Einstein-Hilbert gravity in five dimensions with a negative cosmological constant and a coupling to an axion-dilaton field. It is not clear whether this system might be obtained by some string theory compactification, so that our philosophy in this work is 'bottom-up'. At least, we know some aspects of its holographic dual field theory in some limits. If the GB coupling is set to zero, the dual field theory is known and corresponds to a deformation of $\mathcal{N}=4$ Super Yang-Mills (SYM) theory by a theta-term. On the other hand, the exact field theory dual to pure GB gravity is not currently known, but we know that the theory is dual to a CFT with two different central charges [18-20].

The main goal of this thesis is to find a new solution of the equations of motion representing a black brane with a translationally invariant but anisotropic horizon and then explore the properties of the corresponding dual plasma. The force responsible for keeping the horizon in an anisotropic state is furnished by the axion field, which we take to have a fixed profile in the radial coordinate but to depend linearly in one of the horizon coordinates. This is similar to what has been done in [21] and later in [22,23]. This new solution is interesting from a purely general relativity point of view, for it opens up the possibility to study the thermodynamics of a black brane which depends on several parameters (the temperature, the GB coupling and an anisotropy parameter), presumably giving rise to a rich phase space. In this work we move a first step toward the study of such thermodynamics by computing the boundary stress tensor. This computation requires the machinery of holographic renormalization. More specifically, we use a Hamiltonian approach to the problem, rather than the more commonly used Lagrangian one, in the incarnation of the recursive Hamilton-Jacobi method developed in [24] for the AdSEinstein system with axion-dilaton (without higher derivative corrections). Holographic renormalization of Einstein gravity with the GB term, but without any other field turned on, has been performed in [25].

A more applied motivation for our work is given by the study of the QGP produced in the ultra-relativistic collision of heavy ions at RHIC and LHC. Contrary to naïve expectations, this plasma turns out to be a strongly coupled fluid [26,27]. This fact renders a perturbative approach of limited applicability and motivates the use of the AdS/CFT 
correspondence; see [28] for a review of applications of AdS/CFT to the study of the QGP. One of the diagnostics of the strongly coupled nature of this fluid is represented by 'elliptic flow', i.e. the anisotropic evolution of the fluid in the initial stages before isotropization. Recently, there has been some interest in modeling this anisotropy at strong coupling [22,23] and in studying how various observables may be affected by it. Some of the studies that have been performed include the computation of the shear viscosity to entropy density ratio [29,30], the drag force experienced by a heavy quark [31-33], the energy lost by a quark rotating in the transverse plane [34], the stopping distance of a light probe [35], the jet quenching parameter of the medium [32,36,37], the potential between a quark and antiquark pair, both static [32,36,38,39] and in a plasma wind [38], including its imaginary part [40], Langevin diffusion and Brownian motion [41-43], chiral symmetry breaking [44], the production of thermal photons [45-47] and dileptons [48], and the introduction of a chemical potential [49,50]; see [51] for a review of some of these computations and [52] for similar computations in a fluid with dilaton-driven anisotropy.

In order to achieve a more realistic model of the anisotropic plasma it is obviously important to relax some of the assumptions (like the infinite coupling and infinite number of colors) that go into the simplification of having a classical gravity dual. The GB coupling that we introduce here corresponds to allowing for different central charges, $a \neq c$, in the gauge theory [18-20]. We compute these two central charges for our particular solution, verifying that they are indeed different. On general grounds, looking at how higher derivative terms affect physical observables on the gauge theory might also be useful to constrain the string landscape, e.g. by excluding regions of parameters that would result in pathologies, as advocated for example in [16,53]. As a final, concrete application of our geometry we compute several observables relevant to study of the QGP, namely, the shear viscosity over entropy density ratio, the drag force experienced by a heavy quark moving through the plasma, the jet quenching parameter, the static potential between a quark-antiquark pair (quarkonium) and the photon production rate.

This thesis is organized as follows. Chapter 2 is a standard review of AdS/CFT correspondence. In Chapter 3, we review Lovelock gravity, emphasizing the properties that motivate its study within the context of the AdS/CFT correspondence. Chapter 4 is devoted to a first simple computation in the framework of higher curvature gravity. More precisely, we use GB (and also Quasi-topological) gravity as the holographic setup to compute the so called Chern-Simons diffusion rate of the dual plasma [54]. Chapters 5 and 6 constitute the main results of this thesis $[55,56]$, where we find the anisotropic gravity solution with the GB term and use it as the gravity dual of a strongly coupled anisotropic plasma. Finally, our conclusions are presented in Chapter 7. 


\section{Chapter 2}

\section{AdS/CFT correspondence}

Dualities have taught us a lot over the last centuries [57]. It is surprising when we find two theories, at first not similar to each other, to be just two different descriptions of the same physics. Perhaps this means there is something more fundamental behind it, and it is clearly worth to explore them as much as we can. The AdS/CFT correspondence is one such example of duality and one of the most important discoveries in theoretical physics of the last decades. Maldacena proposed the correspondence in 1997, relating string theory (a theory of gravity) to a gauge theory (without gravity) "living" in the boundary of the space of the gravity theory.

One remarkable aspect of the AdS/CFT is that it is a strong/weak coupling duality. As a consequence, a strongly coupled problem in the gauge theory, which is generally difficult, can be mapped into an easier, weakly coupled problem in the gravity dual theory. The converse is also true, i.e., we can use gauge theories to learn about string theory at strong coupling. In fact, so far string theory is only well defined perturbatively, and we do not know much about non-perturbative string theory. Therefore, we can use the correspondence to actually define what non-perturbative string theory is.

In this Chapter, we motivate the AdS/CFT correspondence by giving heuristic arguments that relate string/gravity theories to gauge theories. We then work on some general aspects of string theory that are necessary to understand the correspondence. Finally, we state the conjecture by equating two different pictures of a system of $N$ coincident D3branes. The classical review about the AdS/CFT is [58], but there are many others at an introductory level; see e.g. [59,60]. There are also some nice recent books about this subject $[61,62]$. 


\subsection{Arguments for plausibility}

In this section we give some heuristic arguments that point the connection of string theory with gauge theories.

\section{'t Hooft large $\mathrm{N}$ expansion}

In the argument proposed by 't Hooft in 1974 [63], we consider a $U(N)$ gauge theory with gauge coupling $g$ and take a particular limit in which $N \rightarrow \infty$ while the parameter $\lambda \equiv g^{2} N$, the 't Hooft coupling, is kept fixed. In this limit the expansion in terms of Feynman diagrams simplifies drastically in such a way that only planar diagrams ${ }^{1}$ contribute. It turns out that in this limit the amplitude expansion for the gauge theory has the same structure of the genus expansion that appears in string theory, suggesting a connection between gauge theory and string theory.

Let us review this argument for the case of a generic adjoint field $\Phi$ in a $U(N)$ gauge theory with Lagrangian given by

$$
\mathcal{L}=\frac{1}{g^{2}} \operatorname{Tr}\left[(\partial \Phi)^{2}+\Phi^{3}+\Phi^{4}+\ldots\right]
$$

Here we are ignoring a possible mass term since it does not change the argument. We emphasize that this argument holds in general, in particular for $S U(N) \mathcal{N}=4$ Super Yang-Mills theory which is the case of interest for the statement of the AdS/CFT correspondence. In the adjoint representation, $\Phi$ can be viewed as $N \times N$ matrix $\Phi_{a}^{b}$, where $a, b=1, \ldots, N$. As we usually do in quantum field theory, we can use Wick's theorem to derive the Feynman rules. The rules are summarized below, where we also introduced the double line notation for convenience.

\footnotetext{
${ }^{1} \mathrm{~A}$ planar diagram is a diagram that can be drawn on the plane without crossing lines. We can think of planar diagrams as those who can be drawn on the surface of a sphere. On the other hand, a non-planar diagram can only be drawn on a torus or a surface of higher genus.
} 
Propagator

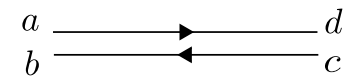

$\sim g^{2} \delta_{a}^{d} \delta_{c}^{b}$

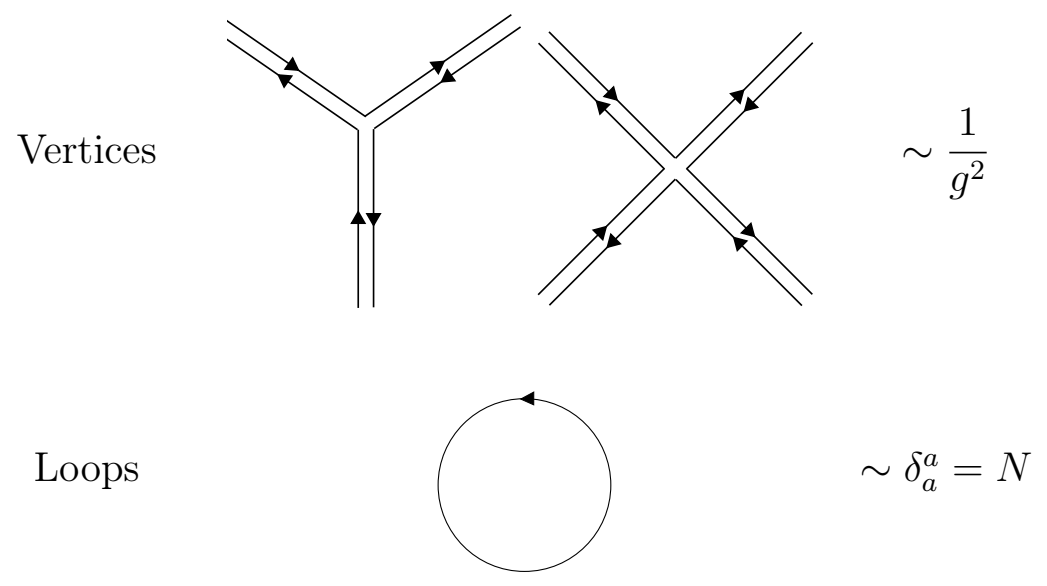

With these rules we can now compute vacuum-to-vacuum amplitudes by summing over all possible diagrams. One way to organize the diagrammatic sum is accordingly to their number of vertices, propagators and loops. Using the Feynman rules, a general diagram with $V$ vertices, $P$ propagators and $L$ loops contributes with

$$
\mathcal{A}(V, P, L) \sim\left(g^{2}\right)^{P}\left(\frac{1}{g^{2}}\right)^{V} N^{L}=\lambda^{P-V} N^{V-P+L},
$$

where $\lambda=g^{2} N$ is the 't Hooft coupling. Recall the Euler characteristic formula for graphs

$$
V-P+L=2-2 h
$$

where $h$ is the genus number, i.e., the number of "holes" (like an $h$-torus). Summing over all connected vacuum-to-vacuum diagrams, we obtain the schematic form for the total amplitude

$$
\sum \mathcal{A}=\sum_{h=0}^{\infty} \sum_{n=0}^{\infty} c_{h, n} \lambda^{n} N^{2-2 h}=\sum_{h=0}^{\infty} f_{h}(\lambda) N^{2-2 h},
$$

where $f_{h}(\lambda)$ is the sum over the diagrams that can be drawn on a surface of genus $h$. In the limit $N \rightarrow \infty$, the sum is clearly dominated by the term $h=0$, which corresponds to the planar diagrams. The higher genus terms are suppressed by powers of $1 / N^{2}$.

This diagrammatic expansion in the gauge theory has the same structure of the vacuum-to-vacuum amplitude of the genus expansion of Riemann surfaces in string theory (Figure 2.1). Basically, strings can split and join into "pairs of pants", where the interaction is controlled by a parameter $g_{s}$, the string coupling constant. 


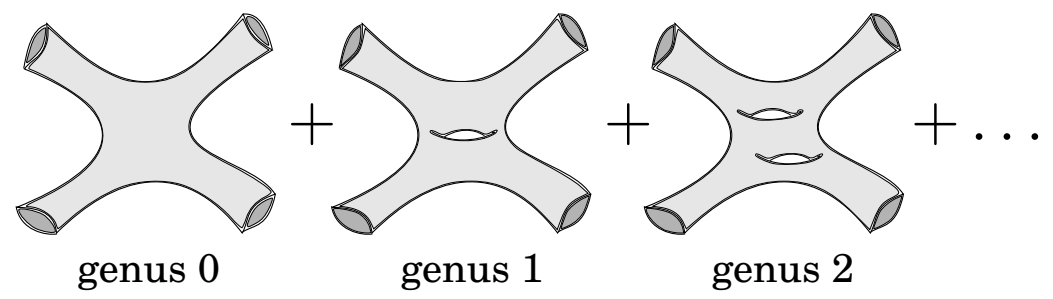

Figure 2.1: Genus expansion for an amplitude with two closed strings going into two closed strings.

\section{Holographic principle}

The AdS/CFT can be viewed as a concrete example of the holographic principle. The history behind the holographic principle begins with the "area theorem" by Hawking in 1971 [64], stating that the area of the horizon of a black hole must always increase. This behavior has an obvious analogy with the second law of thermodynamics, where the entropy of a closed system must always increase. After some years, Bekenstein [65] took a step forward and established that the entropy of the black hole is proportional to the area of the horizon

$$
S_{B H}=\frac{A_{H}}{4} .
$$

As a consequence of this remarkable fact we now should think about black holes as thermodynamical objects. However, there is a subtlety here: the entropy of a black hole scales like area, instead of volume as one could naively expect. Susskind interpreted these results as a holographic principle [66]: for a theory of quantum gravity, such as string theory, the description of a volume of the space is encoded on its boundary, in the same way as a hologram in $2 \mathrm{~d}$ encodes the information of a $3 \mathrm{~d}$ object. Therefore, we expect quantum gravity to have the same number of degrees of freedom (d.o.f.) of a field theory without gravity and black holes, in one lower dimension. Let us check it explicitly for the case of gravity in the Anti-de Sitter space.

\section{Counting degrees of freedom}

Let $N_{\mathrm{QFT}}$ and $N_{\text {Gravity }}$ be the number of degrees of freedom of the quantum field theory (QFT) and gravity theory, respectively. Of course, they are both infinite, so we need to regularize. For a QFT in $d$ spacetime dimensions, we introduce an IR cutoff by putting the theory in a $d-1$ dimensional box of length $\ell$. Also, we put an UV cutoff assuming a lattice site of size $\delta$. Define $N$ as the number of d.o.f per lattice site, so the total number 
of d.o.f. is $N \times$ (\# of states). In this way we have

$$
N_{\mathrm{QFT}}=\left(\frac{\ell}{\delta}\right)^{d-1} N
$$

In the gravity side, the number of degrees of freedom is given by its maximum entropy computed using the area law for a black hole that occupies all the volume

$$
N_{\text {Gravity }}=S_{\max }=\frac{A_{\text {Bdry }}}{4 G_{N}} .
$$

For the Anti-de Sitter space $\left(A d S_{d+1}\right)$ the metric is given by

$$
d s^{2}=\frac{L^{2}}{z^{2}}\left(-d t^{2}+d \vec{x}^{2}+d z^{2}\right)
$$

and the area $A_{\text {bdry }}$ of the black hole, at fixed time, is

$$
A_{\mathrm{Bdry}}=\left.\int d^{d-1} x \sqrt{-\gamma}\right|_{z=0}=\left.\int_{0}^{\ell} d^{d-1} x\left(\frac{L}{z}\right)^{d-1}\right|_{z=\delta}=\left(\frac{\ell L}{\delta}\right)^{d-1} .
$$

Note that we also introduced two cutoffs because the integral is divergent for two reasons: $\left(\frac{L}{z}\right)^{d-1} \stackrel{z \rightarrow 0}{\rightarrow} \infty$ and $\int d^{d-1} x=\infty$. If we identify $N_{\mathrm{QFT}}=N_{\text {Gravity }}$, we obtain the same parametric dependence on the cutoffs, provided the identification

$$
\frac{L^{d-1}}{G_{N}} \sim N
$$

\section{Renormalization group flow}

Quantum field theories are organized in energy scales. In general, the dependence on the energy scale in a quantum field theory with coupling constant $g$ is described by a renormalization group flow equation, ${ }^{2}$ usually called beta function, of the form

$$
\beta(g)=r \frac{d g}{d r}
$$

where $r$ is a parameter related to the energy scale of the theory. In units where $\hbar=c=1$, energy has units of length ${ }^{-1}$, so we need to introduce an extra length scale $L$ such that

$$
E=\frac{r}{L^{2}}
$$

\footnotetext{
${ }^{2}$ For example, the beta in QED function is positive, meaning that the theory is weakly coupled at low energies. On the other hand, in QCD the opposite happens: the beta function is negative and the theory is strongly coupled at low energies. In the special case of a vanishing beta function, the coupling constant does not depend on the energy scale and the theory is said to be conformal.
} 
The previous discussion about the holographic principle suggests the possibility to include an extra dimension, $z$, where gravity lives. We can identify the extra dimension with the inverse of the energy scale, with $L$ being the curvature radius of $A d S$. We just arrived at one of the most intuitive pictures of the AdS/CFT correspondence: the geometrization of the renormalization group flow (Figure 2.2). From the hint of the holographic principle, we can think of the theory of quantum gravity as being equivalent to a quantum field theory, without gravity, living in the boundary of the space, at $z=0$.
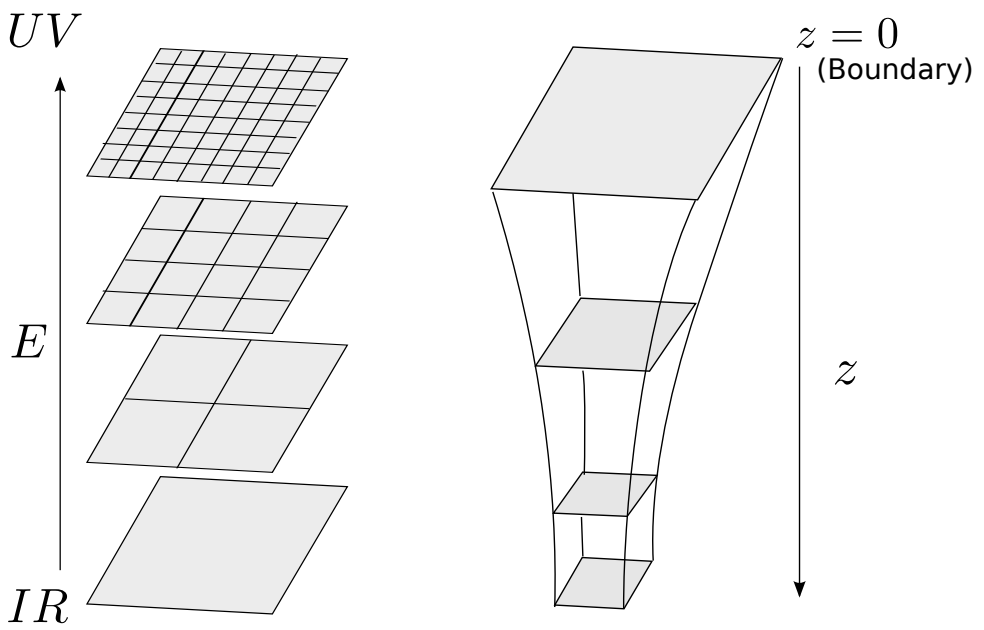

Figure 2.2: Left: the renormalization group flow from the QFT point of view. Right: the energy scale of the QFT identified as an extra dimension z.

\subsection{Basics of string theory}

This section is a very brief overview of string theory. For our purposes to establish the AdS/CFT correspondence, we give emphasis to the construction of the massless spectrum of closed superstrings. This will be important to understand the origin of the fields in type IIB supergravity.

\subsubsection{Generalities}

String theory is a theory of relativistic, quantum, interacting one and higher dimensional objects (strings and branes). It was originally proposed as a theory of the strong interactions, but soon this idea was abandoned due to the success of QCD. Later, it was found that string theory naturally incorporates gravity since a massless spin two state, the graviton, appeared in the spectrum of quantized closed strings. String theory is therefore a theory of quantum gravity, and over the past years it has been viewed as a promising candidate of a theory that unifies all the forces in nature. 
The string length $l_{s}$ is the only fundamental dimensional parameter in the theory, it sets the scale at which string effects become relevant. The string tension is given by

$$
T=\frac{1}{2 \pi \alpha^{\prime}}, \quad \text { where } \quad \alpha^{\prime}=l_{s}^{2}
$$

There is also another parameter, the coupling constant $g_{s}$, that controls the strength of the string interactions. It is important to emphasize that $g_{s}$ is not a fundamental parameter of the theory. ${ }^{3}$ The action for the bosonic string is given by the Nambu-Goto action

$$
S_{\mathrm{NG}}=-T \int d \tau d \sigma \sqrt{-\operatorname{det} g_{\alpha \beta}}, \quad(\alpha, \beta=\tau, \sigma),
$$

where $g_{\alpha \beta}=G_{\mu \nu} \partial_{\alpha} X^{\mu} \partial_{\beta} X^{\nu}$ is the induced metric on the string world-sheet, with $X^{\mu}$ being the embedding coordinates and $G_{\mu \nu}$ the target space metric. The spectrum of bosonic string has a tachyon, an unstable vacuum state with negative mass. Moreover, the spectrum does not contain fermions, which requires the introduction of supersymmetry. Consistency of the theory fixes the spacetime dimension to be 26 in bosonic string, while for the superstring the dimension is fixed to 10 .

\subsubsection{Superstring}

As we mentioned before, bosonic string is well described by the Nambu-Goto action (2.14). However, there is an alternative action equivalent to the Nambu-Goto action, called Polyakov action, that is more suitable to the construction and quantization of the superstring. In the Ramond-Neveu-Schwarz (RNS) formalism of the superstring, we start with the gauge-fixed Polyakov action with the addition of fermionic fields

$$
S=-\frac{T}{2} \int d \tau d \sigma \eta^{\alpha \beta}\left(\partial_{\alpha} \mathcal{X}^{\mu} \partial_{\beta} \mathcal{X}_{\mu}+i \bar{\psi}^{\mu} \gamma_{\alpha} \partial_{\beta} \psi_{\mu}\right)
$$

where $\mathcal{X}^{\mu}(\tau, \sigma)$ and $\psi^{\mu}(\tau, \sigma)$ are the bosonic and fermionic world-sheet fields, respectively. Note that $\mathcal{X}^{\mu}$ are world-sheet scalars and $\psi^{\mu}$ are world-sheet spinors, but from the point of view of the spacetime they are both vectors. This action is invariant under the global, infinitesimal, world-sheet supersymmetry transformations

$$
\delta_{\epsilon} \mathcal{X}^{\mu}=\bar{\epsilon} \psi^{\mu}, \quad \delta_{\epsilon} \psi^{\mu}=-i \rho^{\alpha} \partial_{\alpha} \mathcal{X}^{\mu} \epsilon
$$

\footnotetext{
${ }^{3}$ In fact, it is given by $g_{s}=e_{0}^{\phi}$, the exponential of the asymptotic value of a field of the theory.
} 
where $\epsilon$ is a constant, anti-commuting two-component spinor. The Dirac matrices can be chosen as

$$
\gamma^{0}=\left(\begin{array}{cc}
0 & -i \\
i & 0
\end{array}\right), \quad \gamma^{1}=\left(\begin{array}{cc}
0 & i \\
i & 0
\end{array}\right)
$$

We can choose $\psi^{\mu}$ to be a world-sheet Majorana spinor, i.e., $\psi=\left(\begin{array}{l}\psi_{+} \\ \psi_{-}\end{array}\right)$, with $\psi_{ \pm}$both real. With these choices, the fermionic part of the action becomes

$$
S_{f} \sim \int d \tau d \sigma\left(\psi_{-} \cdot \partial_{+} \psi_{-}+\psi_{+} \cdot \partial_{-} \psi_{+}\right)
$$

where we introduced world-sheet light-cone coordinates $\sigma^{ \pm}=t \pm \sigma$, and $\partial_{ \pm}=\frac{\partial}{\partial \sigma^{ \pm}}$. The equations of motions are

$$
\partial_{-} \psi_{+}^{\mu}=0, \quad \partial_{+} \psi_{-}^{\mu}=0 .
$$

When deriving the equations of motion above, by varying the action w.r.t. the fields, a boundary term arises and we need to impose suitable boundary conditions (b.c.) to eliminate such term. The boundary term of the fermionic part is

$$
\left.\delta S_{f} \sim \int_{\tau_{0}}^{\tau_{1}} d \tau\left(\psi_{+} \cdot \delta \psi_{+}-\psi_{-} \cdot \delta \psi_{-}\right)\right|_{\sigma=0} ^{\sigma=\pi}
$$

We need to treat the cases of open and closed string separately now.

\section{Boundary conditions: open string}

For the open string, the contribution from the two endpoints have to vanish separately. For the endpoint at $\sigma=0$, we can choose without loss of generality

$$
\psi_{+}^{\mu}(\tau, 0)=\psi_{-}^{\mu}(\tau, 0)
$$

This choice still leaves two possibilities at the other string endpoint $(\sigma=\pi)$ :

- $\psi_{+}^{\mu}(\tau, \pi)=+\psi_{-}^{\mu}(\tau, \pi) \quad$ Ramond $(\mathrm{R})$ boundary condition

- $\psi_{+}^{\mu}(\tau, \pi)=-\psi_{-}^{\mu}(\tau, \pi) \quad$ Neveu-Schwarz (NS) boundary condition

With these boundary conditions we can write the mode expansion satisfying the equations of motion and the appropriate boundary condition

$$
\begin{array}{ll}
\psi_{ \pm}^{\mu}(\tau \pm \sigma)=\frac{1}{\sqrt{2}} \sum_{r \in \mathbb{Z}} \psi_{r}^{\mu} e^{-i r(\tau \pm \sigma)} & \text { (R-sector) } \\
\psi_{ \pm}^{\mu}(\tau \pm \sigma)=\frac{1}{\sqrt{2}} \sum_{r \in \mathbb{Z}+1 / 2} \psi_{r}^{\mu} e^{-i r(\tau \pm \sigma)} & \text { (NS-sector) }
\end{array}
$$


Additionally, the Majorana condition requires $\psi_{-r}^{\mu}=\left(\psi_{r}^{\mu}\right)^{*}$. As it happens for the bosonic string, quantization of the superstring requires us to solve some Virasoro constraints. This is best done by introducing spacetime light-cone coordinates, leaving $\psi^{i}(i=1, \ldots, 8)$ as the true degrees of freedom. In the quantization of the superstring, we promote the coefficients $\psi_{r}^{\mu}$ to fermionic operators satisfying anti-commutation relations

$$
\left\{\psi_{r}^{i}, \psi_{s}^{i}\right\}=\delta^{i j} \delta_{r+s, 0} .
$$

Let us now analyze the two different sectors. The NS-sector turns out to be simpler, because there are no zero modes $\psi_{0}^{i}$. We define the NS vacuum $|0\rangle_{N S}$ as the state annihilated by all positive modes $\psi_{r}^{i}$. This state turns out to be tachyonic, with negative mass. Applying each negative modes, we can construct the excited states. The first excited state is massless and is obtained by applying $\psi_{-1 / 2}^{i}|0\rangle_{N S}$. This forms a vector of $S O(8)$ that we will denote by $\boldsymbol{8}_{v}$.

The main difference of the R-sector with respect to the NS-sector is the presence of zero modes $\psi_{0}^{i}$. They do not contribute to the string state energy so the ground state is degenerate. The anti-commutation relations for the zero modes have the form of a Clifford algebra $\left\{\psi_{0}^{i}, \psi_{0}^{j}\right\}=\delta^{i j}$, being a 16-component spinor representation of $S O(8)$ which can be reduced into two 8-component spinor representation of opposite chiralities. Let us denote these representations by $\boldsymbol{8}_{c}$ and $\boldsymbol{8}_{s}$.

The NS-sector brings a tachyon that we want to remove. This is done by introducing the so called Gliozzi-Scherk-Olive (GSO) projection, that not only removes the tachyon but also equates the number of fermionic and bosonic degrees of freedom. Acting on the NS-sector, the GSO projection removes states with even number of fermionic modes. In particular, it removes the tachyonic state $|0\rangle_{N S}$. Acting on the R-sector, it projects out one of the 8's, leaving the one with opposite chirality.

\section{Boundary conditions: closed string}

Roughly speaking, we can think about the closed string as being composed by two copies of the open string, referred to as left and right movers. We can impose the boundary conditions for the closed string in four different ways. For each of the left and right movers we can choose the boundary condition to be either Ramond or Neveu-Schwarz, giving rise to the four sectors of the closed string

$$
\text { (Left b.c. - Right b.c.): (NS-NS), (NS-R), (R-NS), (R-R). }
$$

As it occurred in the open string, we need to apply the GSO projection to remove the tachyon on both left and right movers. Accordingly to the chirality left by the GSO 
projection when acting on the R-sector, we classify the theory as type IIA and type IIB. In type IIB superstring, the right and left movers have the same $\boldsymbol{8}_{c}$ left by the GSO projection, while in the type IIA the right and left movers are left with $\boldsymbol{8}_{c}$ and $\boldsymbol{8}_{s}$, with opposite chiralities. Since we want to focus on type IIB supergravity in the next subsection, we summarize the massless spectrum of type IIB strings in Table 2.1.

Table 2.1: Massless closed string states.

\begin{tabular}{c|c|c|l} 
Sector & |\rangle$_{L} \otimes|\rangle_{R}$ & $S O(8)$ rep & Fields \\
\hline \hline $\mathrm{NS}-\mathrm{NS}$ & $\boldsymbol{8}_{v} \otimes \mathbf{8}_{v}$ & $\mathbf{1}+\mathbf{2 8}_{v}+\mathbf{3 5}_{v}$ & $\phi, B_{\mu \nu}, G_{\mu \nu}$ \\
$\mathrm{NS}-\mathrm{R}$ & $\boldsymbol{8}_{v} \otimes \mathbf{8}_{c}$ & $\mathbf{8}_{s}+\mathbf{5 6}_{s}$ & $\lambda_{\alpha}^{1}, \psi_{\mu \alpha}^{1}$ \\
$\mathrm{R}-\mathrm{NS}$ & $\boldsymbol{8}_{c} \otimes \mathbf{8}_{v}$ & $\mathbf{8}_{s}+\mathbf{5 6}_{s}$ & $\lambda_{\alpha}^{2}, \psi_{\mu \alpha}^{2}$ \\
$\mathrm{R}-\mathrm{R}$ & $\mathbf{8}_{c} \otimes \mathbf{8}_{c}$ & $\mathbf{1}+\mathbf{2 8}_{c}+\mathbf{3 5}_{c}$ & $C_{(0)}, C_{(2)}, C_{(4)}^{+}{ }_{\mu \nu \rho \sigma}$
\end{tabular}

The particle content can be grouped accordingly with their bosonic/fermionic nature and they have the following symmetry properties

$$
\begin{gathered}
\text { Bosons }\left\{\begin{array}{l}
G_{\mu \nu} \quad \text { metric } \\
C_{(0)}+i \phi \quad \text { axion-dilaton } \\
B_{\mu \nu}+i C_{(2)} \text { r } \quad \text { rank } 2 \text { antisymmetric } \\
C_{(4)}^{+} \quad \text { rank } 4 \text { antisymmetric and self-dual }
\end{array}\right. \\
\text { Fermions } \begin{cases}\psi_{\mu \alpha}^{I} & \text { Majorana-Weyl gravitinos } \\
\lambda_{\alpha}^{I} & \text { Majorana-Weyl dilatinos } \quad I=1,2 .\end{cases}
\end{gathered}
$$

It is important to note that in this formalism of the superstring, we started with an action with supersymmetry on the world-sheet, but supersymmetry in the spacetime is not manifest. Moreover, the GSO projection may be somewhat artificial to remove the tachyon and equate the number of bosons and fermions in spacetime. Another formalism of the superstring, the Green-Schwarz (GS) formalism [67], makes spacetime supersymmetry manifest, but the symmetries in the world-sheet are not. As a final comment, there is still another formalism, called pure spinor and developed by Berkovits [68], that combines the advantages of the RNS and GS formalisms, but we will not treat them here.

\subsubsection{Type IIB supergravity}

The reason for us to be focusing on the massless states is that, at low energies, the massive modes of string theory are not excited so we can integrate them out, leaving only the massless fields. Also, if we consider a weakly curved spacetime, we can treat strings 
essentially as point-like objects. In the low energy and low curvature limit, string theory reduces to general relativity coupled to other fields,

$$
S=\frac{1}{16 \pi G_{N}} \int d^{10} x \sqrt{-g}(R+\text { massless modes })
$$

plus some calculable higher curvature corrections. This is the supergravity limit of string theory. For type IIB superstring, the low energy effective action is given by

$$
\begin{aligned}
S_{I I B}= & \frac{1}{32 \pi G_{N}}\left[\int d^{10} x \sqrt{-g} e^{-2 \phi}\left(2 R+8 \partial_{\mu} \phi \partial^{\mu} \phi-\left|H_{(3)}\right|^{2}\right)-\right. \\
& \left.\quad-\int d^{10} x\left(\left|F_{(1)}\right|^{2}+\left|\tilde{F}_{(3)}\right|^{2}+\frac{1}{2}\left|\tilde{F}_{(5)}\right|^{2}\right)-\int C_{(4)}^{+} \wedge H_{(3)} \wedge F_{(3)}\right]+ \text { fermions }
\end{aligned}
$$

where

$$
\begin{aligned}
& F_{(1)}=d C_{(0)}, \quad H_{(3)}=d B_{(2)}, \quad F_{(3)}=d C_{(2)}, \quad F_{(5)}=d C_{(4)}^{+}, \\
& \tilde{F}_{(3)}=F_{(3)}-C_{(0)} \wedge H_{(3)}, \quad \tilde{F}_{(5)}=F_{(5)}-\frac{1}{2} C_{(2)} \wedge H_{(3)}+\frac{1}{2} B_{(2)} \wedge F_{(3)},
\end{aligned}
$$

and $\left|F_{(p)}\right|^{2}=\frac{1}{p !} F_{\mu_{1} \ldots \mu_{p}} F^{\mu_{1} \ldots \mu_{p}}$. The action for type IIB supergravity above is not complete, we still need to impose the self duality condition $F_{(5)}=\star F_{(5)}$ by hand.

\subsection{D-branes: the two pictures}

String theory is not only a theory of strings, but also of branes. A $p$-brane is a $p$ dimensional spatially object. An important class of $p$-branes are $\mathrm{D} p$-branes (D stands for Dirichlet) where open strings can end. The study of branes, more specifically a stack of $N$ coincident D3-branes, was the main insight that led to the AdS/CFT correspondence. In what follows, we study this system of branes from two different perspectives. The AdS/CFT correspondence is, roughly speaking, the statement that both pictures are equivalent.

\subsubsection{Open string picture}

A remarkable property about Dp-branes is that they contain gauge fields living on their world-volume [69]. More precisely, if we quantize an open string ending on a $\mathrm{D} p$-brane, we obtain in the massless modes of the spectrum an abelian gauge field $A_{\mu}(\mu=0,1, \ldots, p)$. For more than a single D-brane, the structure is richer: we can have a string with endpoints 
on the same brane or with endpoints on different branes. In the case of $N$ coincident Dbranes, what happens is that they contain a $S U(N)$ non-abelian gauge theory .

For our purposes, it is convenient to define coordinates along the brane and transverse to that

$$
\begin{array}{ll}
x^{\mu}=\left(x^{0}, x^{1}, \ldots, x^{p}\right) & \text { longitudinal coordinates } \\
y^{i}=\left(x^{p+1}, \ldots, x^{9}\right) & \text { transverse coordinates. }
\end{array}
$$

Note that the presence of a $p$-brane breaks translational invariance in $\mathbb{R}^{9,1}$. In the case of a flat D $p$-brane,

$$
\underbrace{S O(9,1)}_{D=10} \rightarrow \underbrace{S O(p, 1)}_{\text {flat D } p \text {-brane }} \times \underbrace{S O(9-p)}_{\text {transverse space }} .
$$

All fields supported on the D-brane only depend on the $x^{\mu}$ coordinates.

For the particular case of $N$ coincident D3-branes, the massless spectrum is given by a four dimensional gauge field $A_{\mu}$, six scalars $\phi^{i}$ and four Weyl fermions $\chi_{a}$. Surprisingly, in the low energy limit, the effective action for these modes is the same as for $\mathcal{N}=4$ SYM theory with gauge group $S U(N)$ in $d=4$ spacetime dimensions [70]. To give an idea about how this picture emerges, let us remember the action for Yang-Mills theory,

$$
S_{\mathrm{YM}}=\frac{1}{g_{\mathrm{YM}}^{2}} \int d^{4} x F_{\mu \nu} F^{\mu \nu}, \quad F_{\mu \nu}=\partial_{\mu} A_{\nu}-\partial_{\nu} A_{\mu} .
$$

On the other hand, the dynamics of a single D3-brane is governed by the Dirac-BornInfeld-Action (DBI)

$$
S_{\mathrm{DBI}}=T_{D 3} \int d^{4} x e^{-\phi} \sqrt{-\operatorname{det}\left(g_{\mu \nu}+\alpha^{\prime} F_{\mu \nu}\right)}, \quad T_{D 3} \sim \frac{1}{g_{s} l_{s}^{4}},
$$

where here $g_{\mu \nu}$ is the induced metric on the D-brane world-volume. Let us see what happens in the low energy limit, i.e., the limit in which we consider energies much smaller than the energy scale $1 / l_{s}$ of string theory. Expanding to the lowest order in $\alpha^{\prime}$, one term that arises is exactly the Yang-Mills term (2.31), provided the identification

$$
g_{\mathrm{YM}}^{2}=4 \pi g_{s}
$$

The other terms of the low energy expansion correspond to the interactions with closed string modes emitted and absorbed by the brane, and also the interaction of closed strings with themselves. This means that, in total, the action for the coincident branes is effectively described by

$$
S_{\mathrm{SYM}}+S_{\text {closed }}+S_{\text {closed/open }}
$$


The strength of the interaction between closed strings is governed by the Newton constant $G_{N}$. But $G_{N} \sim\left(\alpha^{\prime}\right)^{4}$ in ten dimensions, thus the interaction goes to zero in the low energy limit. The interaction between open and closed strings is also controlled by $G_{N}$ and goes to zero for the same reason. Therefore, in the low energy limit we obtain free closed strings, i.e., free gravity.

However, the interactions for $S_{S Y M}$ are controlled by $g_{\mathrm{YM}}$ and not by $G_{N}$, so they are not turned off in the low energy limit. We conclude that at the end we obtain

$$
S_{\mathrm{SYM}}+\text { free gravity in } \mathbb{R}^{9,1} \text {. }
$$

The next step is to consider another picture and see how we can relate the two pictures.

\subsubsection{Closed string picture}

D-brane can also be viewed as defects in spacetime: since D-branes gravitate, they deform the space around them. We consider type IIB supergravity and we want to find the spacetime metric sourced by $N$ coincident D3-branes. The relevant part of the action for type IIB supergravity is ${ }^{4}$

$$
S_{I I B}=\frac{1}{16 \pi G_{N}} \int d^{10} x \sqrt{-g}\left[e^{-2 \phi}\left(R+4 \partial_{\mu} \phi \partial^{\mu} \phi\right)-\frac{2}{5 !}\left|F_{(5)}\right|^{2}\right]
$$

where $\phi$ is the dilaton scalar field and $F_{(5)}=\star F_{(5)}$ is a self dual 5-form. Remember that branes preserve $S O(p, 1) \times S O(9-p)$ symmetry, so we choose an Ansatz consistently with the desired symmetries (and we set $p=3$ )

$$
\begin{gathered}
d s^{2}=\underbrace{\frac{1}{\sqrt{H(y)}} \eta_{\mu \nu} d x^{\mu} d x^{\nu}}_{S O(3,1)}+\underbrace{\sqrt{H(y)} \sum_{i=1}^{6} d y_{i} d y^{i}}_{S O(6)}, \\
\phi=\phi_{0}=\text { const. }, \quad F_{(5)}=(1+\star) d t \wedge d x^{1} \wedge d x^{2} \wedge d x^{3} \wedge d H^{-1} .
\end{gathered}
$$

Note that the self-duality constraint of $F_{(5)}$ is satisfied by construction. The equation of motion turns out to be of a Laplacian type and the solution reads

$$
H(y)=1+\frac{L^{4}}{y^{4}}
$$

where

$$
L^{4}=4 \pi l_{s}^{4} g_{s} N .
$$

\footnotetext{
${ }^{4}$ The other fields of the type IIB supergravity action (2.27) can be set to zero, consistently with the equations of motion.
} 
Since we are in the supergravity limit, all this computation is valid when $L \gg l_{s}$, i.e., in the point-like limit of the strings. From (2.39), we see that this condition is equivalent to

$$
g_{s} N \gg 1
$$

So far the solution we found for the metric sourced by $N$ coincident D3-branes is

$$
\left(1+\frac{L^{4}}{y^{4}}\right)^{-\frac{1}{2}} \eta_{\mu \nu} d x^{\mu} d x^{\nu}+\left(1+\frac{L^{4}}{y^{4}}\right)^{\frac{1}{2}}\left(d y^{2}+y^{2} d \Omega_{5}^{2}\right)
$$

The parameter $L$ can be considered as the characteristic length scale of the range of the gravitational effects of the D3-branes. For $y \gg L$, we can neglect the $L^{4} / y^{4}$ term above and we obtain flat space. For $y \ll L$, the geometry resembles a "throat" (Figure 2.3). Defining $z=\frac{L^{2}}{y}$ and taking $z \rightarrow \infty$, the geometry inside the throat turns out to be

$$
d s^{2}=\frac{L^{2}}{z^{2}}\left(\eta_{\mu \nu} d x^{\mu} d x^{\nu}+d z^{2}\right)+L^{2} d \Omega_{5}^{2}
$$

where $d \Omega_{5}^{2}$ is the metric of a five-sphere. We recognize this solution as $A d S_{5} \times S^{5}$.

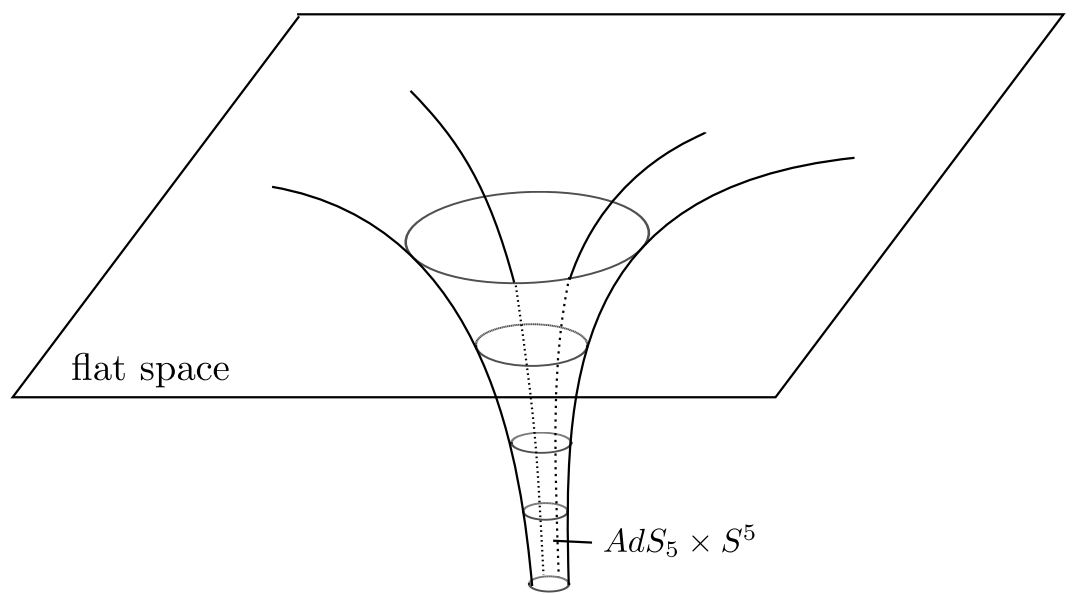

Figure 2.3: The closed string picture: inside the throat, we have interacting closed strings in $A d S_{5} \times S^{5}$. Outside the throat, we have free strings in flat space.

Now we take two limits, the so called Maldacena limit (or near horizon limit):

- First, we take $z \gg L$ (or equivalently $y \ll L$ ). In this case we have the spacetime concentrated inside the throat.

- Then, we keep both $g_{s}$ and $N$ fixed and we take $\alpha^{\prime} \rightarrow 0$. This is again a low-energy limit: the string dynamics in the throat and in the asymptotic flat space decouple. 
After taking these two limits, we end up with free strings in flat space and strings inside the throat. The strings in flat space are free, because as before, the interaction is controlled by $G_{N}$ and it goes to zero in the low energy limit. The strings inside the throat do not have enough energy to climb out, so they decouple from the strings outside the throat. There are still interacting strings, just deep inside of the throat, where the geometry is $A d S_{5} \times S^{5}$. Therefore, in this particular limit, the system in the presence of $N$ coincident D3-brane is described by

$$
\text { Type IIB strings in } A d S_{5} \times S^{5}+\text { free gravity in } \mathbb{R}^{9,1} \text {. }
$$

This is the "closed string picture". It is important to emphasize that this picture is valid for $g_{s} N \gg 1$, while on the other hand the open string picture was valid for $g_{s} N \ll 1$. We are now finally ready to state the conjecture of the AdS/CFT correspondence.

\subsection{Statement of the AdS/CFT correspondence}

To summarize, we have studied two different descriptions of a system of $N$ coincident D3-branes. In the open string picture, D-branes are hyperplanes where open strings can end. The resulting low energy limit is $\mathcal{N}=4 S Y M$ with gauge group $S U(N)$. On the other hand, in the closed string picture, D-branes are viewed as defects in spacetime that deform the geometry around them, and the low energy limit resulted in type IIB strings propagating in $A d S_{5} \times S^{5}$. Note that both descriptions are low energy limits, but there is a difference here: the open string picture holds for $g_{s} N \ll 1$, while in the closed string picture we had $g_{s} N \gg 1$.

The AdS/CFT correspondence is the statement that the two descriptions, besides derived in different regimes of the parameter $g_{s} N$, are in fact equivalent, i.e.,

$$
\mathcal{N}=4 \quad S U(N) \quad S Y M \text { theory = type IIB string theory in } A d S_{5} \times S^{5} .
$$

The relation between the parameters of the two sides are (2.33) and (2.39),

$$
\frac{L^{4}}{l_{s}^{4}}=4 \pi g_{s} N, \quad g_{\mathrm{YM}}^{2}=4 \pi g_{s} .
$$

We still need to specify in which conditions we expect the equivalence to hold. In the strongest form of the conjecture, we assume the equivalence to hold entirely, for full quantum string theory and gauge theory with any value of $N$ and $\lambda$. A more conservative approach is the weaker version of the AdS/CFT correspondence, in which we assume the duality to hold only at the lowest level of the perturbation expansion. In this case we 
identify supergravity, the low energy limit of string theory, with the planar and large $\lambda$ limit of the gauge theory. There is still a mild level of the conjecture, where $N \rightarrow \infty$ but $\lambda$ is kept finite, corresponding to classical string theory. For the special case of large but still finite $\lambda$, we have in the corresponding gravity side $\alpha^{\prime}$ corrections (involving higher order curvature terms) to supergravity. We summarize the different forms of the AdS/CFT correspondence in Table 2.2.

Table 2.2: Three levels of the AdS/CFT correspondence.

\begin{tabular}{c|cclcc} 
& $\mathcal{N}=4 S U(N)$ SYM & & \multicolumn{1}{c}{ IIB theory on $A d S_{5} \times S^{5}$} \\
\hline Strong & $\forall N$ and $\forall g_{\text {YM }}$ & $\Leftrightarrow$ & Full quantum strings & $g_{s} \neq 0, \frac{\alpha^{\prime}}{L^{2}} \neq 0$ \\
\cline { 2 - 6 } Mild & $N \rightarrow \infty, \lambda$ fixed & $\Leftrightarrow$ & Classical strings & $g_{s} \rightarrow 0, \frac{\alpha^{\prime}}{L^{2}} \neq 0$ \\
\cline { 2 - 6 } Weak & $N \rightarrow \infty, \lambda \rightarrow \infty$ & $\Leftrightarrow$ & Classical supergravity & $g_{s} \rightarrow 0, \frac{\alpha^{\prime}}{L^{2}} \rightarrow 0$ \\
\hline
\end{tabular}

An obvious check of the conjecture is to see if the global symmetries on both sides match. The bosonic part of $\mathcal{N}=4 \mathrm{SYM}$ in four dimensions has a conformal symmetry associated to the group $S O(4,2)$; see Appendix A. This matches exactly the isometry group of $A d S_{5}$. The easiest way to see that is to view $A d S_{5}$ as embedded in $\mathbb{R}^{4,2}$. Moreover, the $\mathcal{N}=4$ SYM displays a global $S U(4) R$-symmetry which is the symmetry that rotates the six scalars and the four fermions of the theory. Since $S O(6)_{R} \sim S U(4)_{R}$, this matches the $S O(6)$ symmetry of the $S_{5}$ sphere part of $A d S_{5} \times S_{5}$.

\subsection{Correlation functions}

We also need to provide a prescription to map the observables of the two sides of the duality. The prescription is known as the GKPW (Gubser, Klebanov, Polyakov and Witten) formula and it is a one-to-one field/operator identification, i.e., gauge invariant operators $\mathcal{O}$ of the boundary theory are mapped into bulk fields $\phi$ of the gravity theory. For example, The bulk metric corresponds to the stress energy tensor of the boundary theory. Basically, for each local operator $\mathcal{O}_{i}(x)$ of the gauge theory we add a term to the action

$$
S \rightarrow S+\int d^{4} x \phi_{(0)}^{i}(x) \mathcal{O}(x)
$$

where $\phi_{(0)}^{i}(x)$ is the boundary value of the bulk field $\phi^{i}$ dual to the gauge invariant operator $\mathcal{O}_{i}$. This means that the boundary value of the bulk field acts as a source to the gauge invariant operator of the boundary theory. In the strongest form of the correspondence, we assume the equivalence to hold at the level of the partition function

$$
Z_{\mathrm{SYM}}\left[\phi_{(0)}\right]=Z_{\text {IIB strings }}\left[\phi_{(0)}\right]
$$


The left hand side contains all the information about the gauge theory, since all correlation functions can be computed by taking functional derivatives of the partition function. Going to the weak form of the conjecture by taking the limit $N \rightarrow \infty$ and $\lambda \rightarrow \infty$, we can approximate string theory by supergravity and compute the partition function using saddle point approximation

$$
Z_{\mathrm{SYM}}[J] \simeq e^{-S_{\text {SUGRA }}^{(\text {(on-shell) }}}
$$

In this way the on-shell gravitational action is identified as the generating functional of connected diagrams and correlation functions are extracted via

$$
\left\langle\mathcal{O}_{1}\left(x_{1}\right) \ldots \mathcal{O}_{n}\left(x_{n}\right)\right\rangle_{C F T}=-\left.\frac{\delta^{n} S_{\mathrm{SUGRA}}^{(\text {on-shell })}}{\delta \phi_{(0)}^{1}\left(x_{1}\right) \ldots \delta \phi_{(0)}^{n}\left(x_{n}\right)}\right|_{\phi_{(0)}^{i}=0}
$$

In principle, the extraction of correlation functions seems to be straightforward using the above prescription. However, divergences usually appear in the computation of the onshell gravity action and a regularization procedure is necessary. There are several methods to remove those divergences, which are referredto as holographic renormalization. We will explore one such method in Chapter 5, where we use and extend the Hamilton-Jacobi approach developed in [24]. 


\section{Chapter 3}

\section{Lovelock gravity}

Einstein's general relativity is considered by many as one of the most beautiful theories of physics. The theory was proposed in 1915 to reconcile gravity with the principles of special relativity. The theory relies on the simple, but profound, equivalence principle: locally, we cannot distinguish the effects of gravity from the ones due to an accelerated reference frame. The dynamics are governed by Einstein's equations

$$
\mathcal{G}_{\mu \nu} \equiv R_{\mu \nu}-\frac{1}{2} g_{\mu \nu} R+\Lambda g_{\mu \nu}=8 \pi G_{N} T_{\mu \nu},
$$

where $\mathcal{G}_{\mu \nu}$ is the Einstein tensor. There are some important properties that fully characterize the form of the Einstein's equations above. First, the equations of motion are second order in derivatives, i.e., $\mathcal{G}_{\mu \nu}$ is a function of the metric and its two first derivatives only. Moreover, $\mathcal{G}_{\mu \nu}$ is symmetric and conserved, i.e., $\mathcal{G}_{\mu \nu}=\mathcal{G}_{\nu \mu}$ and $\nabla^{\nu} \mathcal{G}_{\mu \nu}=0$, reflecting the desired properties of the stress tensor $T_{\mu \nu}$ of matter fields. Lovelock's theorem [8] concludes that, in four spacetime dimensions, these conditions are enough to fully fix the form of Einstein's equation. If we go to higher dimensions, however, David Lovelock also showed that we can construct more general symmetric divergence-free tensors yielding second order equations of motion, giving rise to Lovelock gravity.

In this Chapter, we review the general aspects of Lovelock gravity. We define the Lovelock action and find the vacuum solutions of its equations of motion. We focus on the black hole solutions and recent advances regarding Lovelock gravity in the context of the AdS/CFT correspondence. Some of the remarkable results found so far include: the violation of the KSS viscosity bound, the gravity interpretation of the unitarity condition of the CFT, and causality constraints related to the positivity of energy. 


\subsection{Non-coordinate basis}

Lovelock gravity can be described in an elegant and compact way using the language of differential forms. We can construct a non-coordinate basis which provides an orthonormal basis for the tangent space at each point on the manifold,

$$
\mathrm{d} s^{2}=g_{\mu \nu} \mathrm{d} x^{\mu} \mathrm{d} x^{\nu}=\eta_{a b} e^{a} e^{b},
$$

where the vielbein 1-form is defined as $e^{a} \equiv e_{\mu}^{a} \mathrm{~d} x^{\mu}$, so we can rewrite the metric as

$$
g_{\mu \nu}=\eta_{a b} e_{\mu}^{a} e_{\nu}^{b}
$$

Latin indices are called flat or tangent space indices, while Greek indices are called curved or spacetime indices. Another important object is the spin connection 1-form

$$
\omega_{b}^{a}=\omega_{\mu b}^{a} \mathrm{~d} x^{\mu}
$$

Roughly speaking, the spin connection plays the role of the Christoffel symbols of the usual tensorial language. The information about the curvature is entirely encoded into the Cartan's structure equations

$$
\begin{aligned}
& \text { Riemann curvature 2-form: } R^{a b} \equiv \mathrm{d} \omega^{a b}+\omega^{a}{ }_{c} \wedge \omega^{c b} \\
& \text { Torsion 2-form: } T^{a} \equiv \mathrm{d} e^{a}+\omega^{a}{ }_{b} \wedge e^{b}=D e^{a} .
\end{aligned}
$$

Note that $R^{a b}=\frac{1}{2} R^{a b}{ }_{\mu \nu} \mathrm{d} x^{\mu} \wedge \mathrm{d} x^{\nu}$, since it is a 2-form. The relation between the Riemann curvature 2 -form and the Riemann curvature tensor is given by

$$
R_{\mu \nu}^{\lambda \sigma}=R_{\mu \nu}^{a b} e_{a}^{\lambda} e_{b}^{\sigma} .
$$

\subsection{Lovelock action}

Lovelock gravity is the natural generalization of general relativity to more than four spacetime dimensions. In $d$ spacetime dimensions, the action of Lovelock theory is given by a sum of $K \leq\left[\frac{d-1}{2}\right]$ terms, where $K$ is an integer that denotes the highest non-vanishing coefficient $c_{k}$ ( i.e., $c_{k>K}=0$ )

$$
S_{\text {Lovelock }}=\frac{1}{16 \pi G_{N}(d-3) !} \sum_{k=0}^{K} \frac{c_{k}}{d-2 k} \int \mathcal{L}_{k},
$$


where

$$
\mathcal{L}_{k} \equiv \epsilon_{a_{1} \ldots a_{d}} R^{a_{1} a_{2}} \wedge \ldots \wedge R^{a_{2 k-1} a_{2 k}} \wedge e^{a_{2 k+1}} \wedge \ldots \wedge e^{a_{d}}
$$

and $\epsilon_{a_{1} \ldots a_{d}}$ is the totally antisymmetric tensor. The parameters $\left\{c_{k}\right\}$ are the couplings of the theory. To gain some intuition, let us see the first terms of the action explicitly:

- The term $k=0$ has no curvature term, it is simply a term proportional to the element volume: the cosmological constant;

- The term $k=1$ includes one power of the curvature 2-form. The choice $c_{0}=\frac{1}{L^{2}}$ and $c_{1}=1$ results in the usual Einstein-Hilbert action with negative cosmological constant $\Lambda=-\frac{(d-1)(d-2)}{2 L^{2}}$;

- The term $k=2$ gives the so called Gauss-Bonnet (GB) term

$$
\mathcal{L}_{G B}=R^{2}-4 R_{\mu \nu} R^{\mu \nu}+R_{\mu \nu \rho \sigma} R^{\mu \nu \rho \sigma}
$$

Lovelock theories involve higher curvature terms in the Lagrangian, but are constructed in a special way such that the equations of motion are still second order in derivatives so to avoid pathologies such as Boulware-Deser ghosts [10]. Indeed, the absence of ghosts, together with the fact that curvature squared terms appeared in the low energy limit of heterotic string, as pointed out by Zwiebach [9], motivated the earlier studies of Lovelock gravity in string theory.

One interesting feature of the action (3.8) is its connection to topological quantities known as Euler densities. In fact, one recognizes the Lagrangian densities $\mathcal{L}_{k}$ as the higher dimensional extensions of the Euler densities $E_{2 k}$ that appear in the remarkable Gauss-Bonnet theorem, that connects the Riemann curvature, a geometric quantity, to a topological quantity known as the Euler characteristic; see for example [71].

For manifolds with a boundary, as it will be the case of interest in the next chapters, the action (3.8) has to be supplied with boundary terms analogue to the Gibbons-Hawking term, necessary in order to have a well posed variational problem. This is due to the fact that, when varying the action w.r.t. the metric field, a boundary term containing the normal derivative of the metric variation arises and, since we want to fix only the metric variation at the boundary and not its normal derivative, a boundary term has to be added to cancel such contribution. It was found in [72] that the necessary boundary terms are exactly the higher dimensional extensions of the boundary terms of the generalized Gauss-Bonnet theorem for manifolds with a boundary.

Let us now derive the equations of motion and check that they are indeed of second order. 


\subsection{Equations of motion and vacuum solutions}

The equations of motions are derived by extremizing the action (3.8). We will apply the first order formalism, in which we assume the vielbein and the spin connection as independent variables, so we need to vary the action with respect to both vielbein and spin connection, obtaining two equations. Before we do this computation, it is convenient to introduce the notation

$$
\begin{aligned}
R^{a_{1} \ldots a_{2 n}} & \equiv R^{a_{1} a_{2}} \wedge \ldots \wedge R^{a_{2 n-1} a_{2 n}}, \\
e^{a_{1} \ldots a_{n}} & \equiv e^{a_{1}} \wedge \ldots \wedge e^{a_{n}} .
\end{aligned}
$$

First, varying the action with respect to the spin connection, we obtain

$$
\begin{aligned}
\delta_{\omega} \mathcal{L}_{k} & =\epsilon_{a_{1} \ldots a_{d}} \delta\left(R^{a_{1} \ldots a_{2 k}}\right) \wedge e^{a_{2 k+1} \ldots a_{d}} \\
& =\epsilon_{a b \ldots a_{d}} k \delta\left(R^{a b}\right) R^{a_{3} \ldots a_{2 k}} \wedge e^{a_{2 k+1} \ldots a_{d}} \\
& =\epsilon_{a b \ldots a_{d}} k D\left(\delta \omega^{a b}\right) R^{a_{3} \ldots a_{2 k}} \wedge e^{a_{2 k+1} \ldots a_{d}} .
\end{aligned}
$$

Integrating by parts and using the fact that the boundary term does not contribute to the equations of motion,

$$
\begin{aligned}
\delta_{\omega} \mathcal{L}_{k} & =-\epsilon_{a b \ldots a_{d}} k\left(\delta \omega^{a b}\right) D\left(R^{a_{3} \ldots a_{2 k}} \wedge e^{a_{2 k+1} \ldots a_{d}}\right) \\
& =-\epsilon_{a b \ldots a_{d}} k\left(\delta \omega^{a b}\right) R^{a_{2} \ldots a_{2 k}} \wedge D\left(e^{a_{2 k+1} \ldots a_{d}}\right),
\end{aligned}
$$

where we used $D R^{c d}=0$ (Bianchi identity). We note that $D\left(e^{a_{2 k+1} \ldots a_{d}}\right)$ is proportional to the torsion. Thus, we can safely impose the torsion to vanishes as in standard Einstein's gravity. On the other hand, varying the action with respect to the vielbein,

$$
\begin{aligned}
\delta_{e} \mathcal{L}_{k} & =\epsilon_{a_{1} \ldots a_{d}} R^{a_{1} \ldots a_{2 k}} \wedge \delta \underbrace{\left(e^{a_{2 k+1} \ldots a_{d}}\right)}_{d-2 k \text { terms }} \\
& =\epsilon_{a_{1} \ldots a_{d-1} a} R^{a_{1} \ldots a_{2 k}}(d-2 k) \wedge e^{a_{2 k+1} \ldots a_{d-1}} \delta e^{a} .
\end{aligned}
$$

Setting the variation to zero, the equation of motion we obtain is

$$
\mathcal{E}_{a}=\epsilon_{a_{1} \ldots a} \sum_{k=1}^{K} c_{k}\left(R^{a_{1} \ldots a_{2 k}} \wedge e^{a_{2 k+1} \ldots a_{d-1}}\right)=0
$$


The above expression is a polynomial of degree $K$ in the Riemann curvature 2-form. Therefore, we can rewrite the equation as

$$
\mathcal{E}_{a}=\epsilon_{a_{1} \ldots a} \mathcal{F}_{(1)}^{a_{1} a_{2}} \wedge \ldots \wedge \mathcal{F}_{(K)}^{a_{2 K-1} a_{2 K}} \wedge e^{a_{2 K+1} \ldots a_{d-1}}=0
$$

where $\mathcal{F}_{(i)}^{a b} \equiv R^{a b}-\Lambda_{i} e^{a} \wedge e^{b}$, with $\Lambda_{i}$ 's as functions of the couplings $\left\{c_{k}\right\}$. We can think of the $\Lambda_{i}$ 's as effective cosmological constants. We immediately see that the equation (3.17) admits vacuum solutions of form

$$
\mathcal{F}_{(i)}^{a b}=R^{a b}-\Lambda_{i} e^{a} \wedge e^{b}=0, \quad i=1, \ldots, K
$$

Each solution $\Lambda_{i}$ corresponds to a different vacuum, which can be positive, negative or zero (dS, AdS or flat). In view of the AdS/CFT correspondence, we are particularly interested in solutions with negative effective cosmological constant.

\subsection{Black hole solutions}

There are many known black hole solutions in Lovelock gravity; see, e.g., [13,73]. In particular, Lovelock theories admit a large class of asymptotically AdS black hole solutions which are specially interesting in view of the AdS/CFT correspondence. Let us discuss the simplest solution of static spherically symmetric black holes. It is convenient to introduce the polynomial

$$
\Upsilon[\Lambda] \equiv \sum_{k=0}^{K} c_{k} \Lambda^{k}
$$

Plugging (3.18) into the equation of motion (3.16), we see that the effective cosmological constants $\Lambda_{i}$ are the roots of $\Upsilon[\Lambda]$, i.e.,

$$
\Upsilon[\Lambda]=c_{K} \prod_{i=1}^{K}\left(\Lambda-\Lambda_{i}\right)
$$

We choose an Ansatz for the metric of the form

$$
d s^{2}=-N_{\#} f(r) d t^{2}+\frac{d r^{2}}{f(r)}+\frac{r^{2}}{L^{2}} d \Sigma_{d-2, \sigma}^{2}, \quad d \Sigma_{d-2, \sigma}^{2} \equiv \frac{d \rho^{2}}{1-\sigma \frac{\rho^{2}}{L^{2}}}+\rho^{2} d \Omega_{d-3}^{2},
$$

where $d \Omega_{d-3}^{2}$ is the unit $(d-3)$-sphere and $\sigma$ parameterizes the different horizon topologies $(\sigma=+1 \rightarrow$ spherical, $\sigma=0 \rightarrow$ planar, $\sigma=-1 \rightarrow$ hyperbolic). An appropriate choice of vielbein is

$$
e^{0}=N_{\#} \sqrt{f(r)} d t, \quad e^{1}=\frac{1}{\sqrt{f(r)}} d r, \quad e^{a}=\frac{r}{L} \tilde{e}^{a},
$$


where $a=2, \ldots, d-1$ and $\tilde{e}^{a}$ is an vielbein for $d \Sigma_{d-2, \sigma}^{2}$ which explicitly form will not be relevant here. Using the second Cartan equation $\mathrm{d} e^{a}=-\omega^{a}{ }_{b} \wedge e^{b}$, valid in the absence of torsion, we can determine the spin connection 1-form and compute the Riemann curvature components

$$
\begin{aligned}
R^{01} & =-\frac{1}{2} f^{\prime}(r) e^{0} \wedge e^{1}, & R^{0 a} & =-\frac{f^{\prime}(r)}{2 r} e^{0} \wedge e^{a}, \\
R^{1 a} & =-\frac{f^{\prime}(r)}{2 r} e^{1} \wedge e^{a}, & R^{a b} & =-\frac{f(r)-\sigma}{r^{2}} e^{a} \wedge e^{b} .
\end{aligned}
$$

Substituting into the equation of motion results in

$$
\mathcal{E}_{0}=0 \Rightarrow \sum_{k=0}^{K} c_{k} \frac{(-1)^{k}}{r^{2 k}}\left(r\left(f^{k}\right)^{\prime}+(d-2 k-1)(f-\sigma)^{k}\right)=0 .
$$

This equation can be rewritten as

$$
\left[r \frac{d}{d r}+(d-1)\right] \sum_{k=0}^{K} c_{k}\left(\frac{\sigma-f(r)}{r^{2}}\right)^{k}=0
$$

The integration is straightforward and the solution for $f(r)$ is obtained implicitly via a polynomial equation

$$
\Upsilon\left[\frac{\sigma-f(r)}{r^{2}}\right]=\sum_{k=0}^{K} c_{k}\left(\frac{\sigma-f(r)}{r^{2}}\right)^{k}=\frac{\kappa}{r^{d-1}}
$$

where $\kappa$ is a integration constant related to the ADM mass [74]. The position of the horizon $r_{+}$is determined using the fact that $f\left(r_{+}\right)$must vanish. This fixes the solution entirely, except by $N_{\#}$ that remains arbitrary from this analysis. The value of $N_{\#}$ can be fixed, e.g., by imposing the velocity of the speed of light to be unity at the boundary.

\subsection{Gauss-Bonnet black holes}

To be concrete, let us particularize the general solution found for Lovelock black holes in the previous section to the case of five-dimensional Gauss-Bonnet gravity. With appropriate choices of the constants $c_{0}, c_{1}$ and $c_{2}$, the action for Gauss-Bonnet gravity can be written as

$$
S=\frac{1}{16 \pi G_{N}} \int d^{5} x \sqrt{-g}\left(R+\frac{12}{L^{2}}+\frac{L^{2}}{2} \lambda_{\mathrm{GB}}\left(R^{2}-4 R_{\mu \nu} R^{\mu \nu}+R_{\mu \nu \rho \sigma} R^{\mu \nu \rho \sigma}\right)\right),
$$


where $\lambda_{\mathrm{GB}}$ is referred to as the (dimensionless) Gauss-Bonnet coupling. Choosing a planar horizon topology for simplicity, the polynomial (3.27) in this particular case reduces to a quadratic polynomial in $f(r)$ and the solution can be given explicitly by

$$
f(r)=\frac{r^{2}}{L^{2}} \frac{1}{2 \lambda_{G B}}\left(1-\sqrt{1-4 \lambda_{G B}\left(1-\frac{r_{+}^{4}}{r^{4}}\right)}\right) .
$$

In this case we chose the root which is known to be smoothly connected with the EinsteinHilbert action in the limit $\lambda_{\mathrm{GB}} \rightarrow 0$. The other root is known to be unstable [10]. The black hole solution reads

$$
d s^{2}=-f(r) N_{\#}^{2} d t^{2}+\frac{d r^{2}}{f(r)}+\frac{r^{2}}{L^{2}}\left(d x^{2}+d y^{2}+d z^{2}\right) .
$$

Note that

$$
f(r) \stackrel{r \rightarrow \infty}{\rightarrow} \frac{r^{2}}{a^{2} L^{2}}, \quad \text { with } \quad a^{2} \equiv \frac{1}{2}\left(1+\sqrt{1-4 \lambda_{\mathrm{GB}}}\right)
$$

This means that the radius of the asymptotic $A d S$ space of this geometry is $a L$. We can choose $N_{\#}=a$ to fix the boundary speed of light to unity.

\subsection{Lovelock and AdS/CFT correspondence}

Supergravity is the low energy limit of string theory and higher curvature terms appear as $\alpha^{\prime}$ corrections to this limit which correspond, in the dual gauge theory side, to finite 't Hooft coupling corrections. It is not clear if Lovelock gravity arises as a low energy limit of a string theory. The reason for us to consider Lovelock gravity within the context of the AdS/CFT correspondence is because of its simplicity, using it as a simple model to perhaps uncover some universal properties. For example, Lovelock theories represent a nice framework to learn about higher dimensional CFTs, which are still a not well understood subject. It is also important to remember that the Lovelock terms may still appear as the $\alpha^{\prime}$ correction of some string theory due to the vast string landscape.

Many interesting results were obtained in the last few years regarding Lovelock gravity and the AdS/CFT; see, for example, [13,14,17] for a review. Some of the remarkable conclusions obtained so far include:

- The KSS bound of the shear viscosity over entropy density can be violated for Gauss-Bonnet gravity and other higher curvature gravity theories;

- The requirement that Boulware-Deser instabilities (i.e., gravitons propagating with kinetic terms of wrong sign) are absent in Lovelock gravity is equivalent to the 
positivity of the central charge, which corresponds to the condition of unitarity of the dual CFT;

- The constraints that arise by imposing no causality violation in the boundary theory are the same constraints that arise by demanding positivity of the energy flux in the dual CFT.

In the last part of this chapter we will review the above conclusions in more detail.

\subsubsection{Violation of the KSS bound}

Amongst numerous results in the study of strongly coupled systems obtained via the AdS/CFT duality, one of the most important is the computation of the ratio of the shear viscosity over entropy density $\eta / s$. In quantum field theory, the shear viscosity is computed by means of the Kubo formula

$$
\eta=-\lim _{\omega \rightarrow 0} \frac{1}{\omega} \operatorname{Im} G_{i j, i j}^{R}(\omega, \vec{q}=0)
$$

where $G^{R}$ is the retarded Green's function for the energy-momentum tensor

$$
G_{\mu \nu, \alpha \beta}^{R}(q)=-i \int d^{4} x e^{-i q x} \theta(t)\left\langle\left[T_{\mu \nu}(x), T_{\alpha \beta}(0)\right]\right\rangle, \quad q=(\omega, \vec{q}) .
$$

The shear viscosity can be computed holographically using the prescription of [75], and early results led Kovtun, Son and Starinets (KSS) to conjecture a universal lower bound for the shear viscosity over entropy density ratio [76]

$$
\frac{\eta}{s} \geq \frac{1}{4 \pi}
$$

The violation of the KSS bound was found some years later for Gauss Bonnet gravity, where the result was found to be

$$
\frac{\eta}{s}=\frac{1}{4 \pi}\left(1-4 \lambda_{G B}\right)
$$

which violates the bound (3.34) for positive values of $\lambda_{G B}$.

\subsubsection{Unitarity of the dual CFT}

Conformal symmetry is a powerful symmetry that highly limit the form of correlation functions in a CFT. In fact, the two and three-point functions are fully fixed up to a few parameters. For example, the two-point function of the stress tensor of a CFT in $(d-1)$ 
spacetime dimensions is constrained to have the form

$$
\left\langle T_{a b}(x) T_{c d}(0)\right\rangle=\frac{C_{T}}{x^{2(d-1)}} \mathcal{I}_{a b, c d}(x),
$$

where $C_{T}$ is known as the central charge and the explicit form of the structure $\mathcal{I}_{a b, c d}(x)$ will not be important for our discussion. The important point here is the observation that requiring unitarity of the $\mathrm{CFT}$ implies $C_{T}$ to be positive. On the gravity dual side, the unitarity condition is translated into avoiding the so called Boulware-Deser instabilities [10] as we shall explain in more detail below.

The holographic dictionary relates the stress tensor with fluctuations around the metric background $g_{\mu \nu} \rightarrow g_{\mu \nu}+h_{\mu \nu}$. Following the same notation as in Section 3.3, let us assume without loss of generality that the $A d S$ background has an associated cosmological constant $\Lambda_{1}$. Since we are interested in a two-point function, we need to expand the action up to second order in the metric fluctuation, and then evaluate the on-shell action. The two-point function is extracted by taking two functional derivatives of the on-shell action.

In the vielbein formalism, the first variation of the action gives $\delta S_{\text {Lovelock }}=\int\left(\mathcal{E}_{a} \delta e^{a}\right)$, where $\mathcal{E}_{a}$ is the equation of motion (3.16). Taking the second variation, we have

$$
\delta^{2} S_{\text {Lovelock }}=\int\left(\mathcal{E}_{a} \delta^{2} e^{a}+\frac{\partial \mathcal{E}_{a}}{\partial e^{b}} \delta e^{b} \wedge \delta e^{a}\right)
$$

The first term vanishes on-shell since it is simply the equation of motion. In the second term, the derivative acting on $\mathcal{E}_{a}$ will supposedly give rise to many terms, but almost all of them are proportional to $\mathcal{F}_{(1)}^{a b}=R^{a b}-\Lambda_{1} e^{a} \wedge e^{b}$ and they vanish when evaluated on-shell. The only non-vanishing contribution comes from the derivative acting on $\mathcal{F}_{1}^{a b}$. Therefore, evaluating on-shell, we have

$$
\delta^{2} S_{\text {Lovelock }}=\prod_{k \neq 1}^{K}\left(\Lambda_{1}-\Lambda_{k}\right) \int \epsilon_{a a_{1} \ldots a_{d-1}} \frac{\partial \mathcal{F}_{(1)}^{a_{1} a_{2}}}{\partial e^{b}} \wedge e^{a_{3} \ldots a_{d-1}} \wedge \delta e^{b} \wedge \delta e^{a} .
$$

We recognize the overall factor above as $\Upsilon^{\prime}\left[\Lambda_{1}\right]$, with $\Upsilon$ defined in (3.27), and the term inside the integral is simply the second variation of the Einstein-Hilbert action, so that

$$
\delta^{2} S_{\text {Lovelock }}=\Upsilon^{\prime}\left[\Lambda_{1}\right] \delta^{2} S_{\mathrm{EH}}
$$

We see that, in order to the solution of the Lovelock action be indeed a minimum, we need to impose $\Upsilon^{\prime}\left[\Lambda_{1}\right]>0$, so avoiding gravitons propagating with the wrong sign of the kinetic energy. These are known as the Boulware-Deser instabilities [10]. The positivity of $\Upsilon^{\prime}\left[\Lambda_{1}\right]$ 
has been proven in [15] for the EH-branch of solutions of Lovelock gravity. ${ }^{1}$ For example, when we described the Gauss-Bonnet solution in Section 3.5 we had two possible solutions and the one we picked was indeed the one that avoided the Boulware-Deser instability.

From the above analysis, the second order on-shell action is simply the same as in the Einstein-Hilbert case [77] up to a factor $\Upsilon^{\prime}\left[\Lambda_{1}\right]$. The central charge can be read off to be

$$
C_{T}=\frac{d}{2(d-2)} \frac{\Gamma(d)}{\pi^{\frac{d-1}{2}} \Gamma\left(\frac{d-1}{2}\right)} \frac{\Upsilon^{\prime}\left[\Lambda_{1}\right]}{\left(-\Lambda_{1}\right)^{\frac{d-2}{2}}} .
$$

Therefore, we learned that

$$
C_{T}>0 \Leftrightarrow \Upsilon^{\prime}\left[\Lambda_{1}\right]>0
$$

reflecting the identification between avoiding Boulware-Deser instabilities and requiring unitarity of the CFT boundary theory.

\subsubsection{Positivity of the energy flux}

Let us define the energy flux operator

$$
\mathcal{E}(\mathbf{n}) \equiv \lim _{r \rightarrow \infty} r^{d-3} \int_{-\infty}^{\infty} d t n^{i} T_{i}^{0}(t, r \mathbf{n})
$$

where $\vec{n}$ is a unity vector characterizing the direction of flux measurement. Let $\mathcal{O}$ be a given local gauge invariant operator. The expectation value of the energy flux on an initial state $\mathcal{O}|0\rangle$ created by $\mathcal{O}$ is given by

$$
\langle\mathcal{E}(\mathbf{n})\rangle_{\mathcal{O}}=\frac{\left\langle 0\left|\mathcal{O}^{\dagger} \mathcal{E}(\mathbf{n}) \mathcal{O}\right| 0\right\rangle}{\left\langle 0\left|\mathcal{O}^{\dagger} \mathcal{O}\right| 0\right\rangle}
$$

Intuitively, we can imagine that the operator $\mathcal{O}$ creates a localized perturbation at $r=0$ that propagates in spacetime. Then we can think of $\langle\mathcal{E}(\mathbf{n})\rangle_{\mathcal{O}}$ as the energy associated to the perturbation deposited in a calorimeter located far away (at $r=\infty$ ) along the direction $\mathbf{n}$. It is therefore reasonable to expect this quantity to be positive. Here, we will be particularly interested in the case where the operator $\mathcal{O}$ is the stress tensor itself, i.e., $\mathcal{O}=T^{i j} \epsilon_{i j}$, where $\epsilon_{i j}$ is a symmetric traceless polarization tensor. So the expectation value of the energy flux can be seen as the ratio of a three-point correlator over a twopoint correlator of the stress tensor. As we discussed in the previous section, conformal symmetry highly constrains the form of the correlators in a CFT. Together with rotational symmetry, the expectation value of the energy flux in a $(d-1)$ dimensional CFT is almost

\footnotetext{
${ }^{1} \mathrm{By}$ EH-branch we mean the solution that is smoothly connected to the corresponding EH solution obtained by setting the other Lovelock couplings to zero.
} 
fixed up to coefficients $t_{2}$ and $t_{4}$, with

$$
\langle\mathcal{E}(\mathbf{n})\rangle_{T^{i j} \epsilon_{i j}}=\frac{E}{\Omega_{d-3}}\left[1+t_{2}\left(\frac{n_{i} n_{j} \epsilon_{i k}^{*} \epsilon_{j k}}{\epsilon_{i k}^{*} \epsilon_{i k}}-\frac{1}{d-2}\right)+t_{4}\left(\frac{\left|n_{i} n_{j} \epsilon_{i j}\right|^{2}}{\epsilon_{i k}^{*} \epsilon_{i k}}-\frac{2}{d(d-2)}\right)\right]
$$

If we now impose the positivity of the energy flux for any direction $\mathbf{n}$ and polarization $\epsilon_{i j}$, the coefficients $t_{2}$ and $t_{4}$ are constrained. The constraints can be obtained by fixing the unit vector to be $n^{i}=(1,0, \ldots, 0)$ and organizing the polarization tensor $\epsilon_{i j}$ accordingly to their $S O(d-3)$ rotational symmetry around $\mathbf{n}$. We obtain

- tensor (helicity 2), e.g., $\epsilon_{23}=\epsilon_{32}=a$ and all other components vanish,

$$
1-\frac{1}{d-2} t_{2}-\frac{2}{d(d-2)} t_{4} \geq 0
$$

- vector (helicity 1), e.g., $\epsilon_{12}=\epsilon_{21}=a$ and all other components vanish,

$$
\left(1-\frac{1}{d-2} t_{2}-\frac{2}{d(d-2)} t_{4}\right)+\frac{1}{2} t_{2} \geq 0
$$

- $\operatorname{scalar}($ helicity 0$)$, e.g., $\epsilon_{i j}=a \times \operatorname{diag}(-(d-3), 1, \ldots, 1)$,

$$
\left(1-\frac{1}{d-2} t_{2}-\frac{2}{d(d-2)} t_{4}\right)+\frac{d-3}{d-2}\left(t_{2}+t_{4}\right) \geq 0
$$

\subsubsection{Causality violation}

Changing to the gravity point of view, let us see how to connect the positivity of the energy flux to preventing causality violation. In higher curvature gravity, gravitons do not propagate accordingly to the background metric, i.e., they do not follow geodesics, instead they feel an effective background related to their equations of motion. In this analysis, we will be concerned about gravitons emitted from the AdS boundary entering into the bulk and bouncing back to the boundary.

It is important to emphasize what kind of causality violation we are referring to. As we will see, when the graviton emitted towards the bulk goes back to the boundary, it may return at some different point such that it lies outside its own light-cone. From the point o view of the boundary theory, the graviton suddenly "pops out" its light-cone, corresponding to a microcausality violation.

Brigante et al [78] found a first evidence for the possibility of causality violation in higher curvature gravity, where they studied metric perturbations around five-dimensional black holes in Gauss-Bonnet gravity. They found that for values of the Gauss-Bonnet coupling $\lambda_{\mathrm{GB}} \geq 9 / 100$, the perturbations propagate with velocity higher than the speed of 
light from the point of view of the boundary theory. Subsequent works generalized this result. Hofman [79] argued that the study of causality violation goes beyond the context of black holes, and proposed the study of causality in shock wave backgrounds. Then, a lower bound for the Gauss-Bonnet coupling was found and the result was also extended to arbitrary dimensions. Finally, Camanho and Edelstein [15] extended the analysis to Lovelock gravity in arbitrary dimensions and they found more general constraints, matching precisely the ones derived from the positivity of the energy flux analysis.

Let us give a general idea about how these constraints were derived using shock waves. Shock waves are a rich class of solutions of gravity, they have the interesting property of not suffering corrections from higher curvature terms and are known to be exact solutions of string theory [80]. The relevant shock wave background is given by

$$
d s_{\text {shock }}^{2}=d s_{A d S}^{2}+\delta(u) \varpi_{0} z^{d-3} d u^{2}
$$

where $z$ is the radial direction of $A d S$ and we have defined light-cone coordinates $u=$ $t+x^{d-1}$ and $v=t-x^{d-1}$. This solution corresponds to a localized shockwave at $u=0$.

Before dealing with the problem of causality, let us just mention that the coefficients $t_{2}$ and $t_{4}$ appearing in (3.44) can be extracted holographically. Considering fluctuations around the shock wave and finding the cubic term of the on-shell action in the fluctuation, the values found were [15]

$$
t_{2}=\frac{2(d-1)(d-2)}{(d-3)(d-4)} \frac{\Lambda_{*} \Upsilon^{\prime \prime}\left[\Lambda_{*}\right]}{\Upsilon^{\prime}\left[\Lambda_{*}\right]}, \quad t_{4}=0 .
$$

Now we consider the scattering of a graviton with the shock wave profile. The equation of motion for a helicity 2 metric fluctuation $\phi \equiv h_{23}$ is

$$
\partial_{u} \partial_{v} \phi-\varpi_{0} \Lambda_{1} \delta(u) z^{d-1} \mathcal{N}_{2} \partial_{v}^{2} \phi=0, \quad \text { where } \quad \mathcal{N}_{2} \equiv 1-\frac{1}{d-2} t_{2}
$$

Away from $u=0$, the delta function vanishes and the equation of motion reduces to the wave equation $\partial_{u} \partial_{v} \phi=0$, so the solution has the form of a wave packet with definite momentum on both sides. The above equation can be integrated over the delta function to give us a relation between the solution in the two sides $(u<0$ and $u>0)$ of the shock wave

$$
\phi_{>}=\phi_{<} e^{-i P_{v} \varpi_{0} \Lambda_{1} z^{d-1} \mathcal{N}_{2}},
$$

where $P_{v}=-i \partial_{v}$. A relation between the shift in the momentum can be derived acting with $P_{z}=-i \partial_{z}$, so

$$
P_{z}^{>}=P_{z}^{<}-(d-1) P_{v} \varpi_{0} \Lambda_{1} z^{d-2} \mathcal{N}_{2}
$$


For a particle going inside $A d S$, we have $P_{z}^{<}>0$. The perturbation will come back to the boundary if its radial momentum changes sign. This condition is satisfied if

$$
P_{v} \varpi \Lambda_{1} z^{d-2} \mathcal{N}_{2}<0
$$

Now note that $P_{v}=-\frac{1}{2} P^{u}<0$, since $P^{u}$ is related to the energy that has to be positive. Since $\Lambda_{1}$ is of course negative, the gravitons go back to the boundary when

$$
\mathcal{N}_{2}<0
$$

and from (3.51) we read the change in the $v$ coordinate to be

$$
\Delta v=-\varpi_{0} \Lambda_{1} z^{d-1} \mathcal{N}_{2}<0
$$

In this case the graviton goes back to the boundary outside its light-cone, so we conclude that $\mathcal{N}_{2} \geq 0$ is a necessary condition in order to avoid causality violation (Figure 3.1). ${ }^{2}$ We can work out the same analysis for the other helicities, finding analogous constraints. In the end, the constraints obtained in this analysis are exactly the same obtained by imposing the positivity of the energy flux in the previous section. In the end of the day, we discover that the condition to avoid causality violation corresponds to impose the positivity of energy flux on the dual CFT.

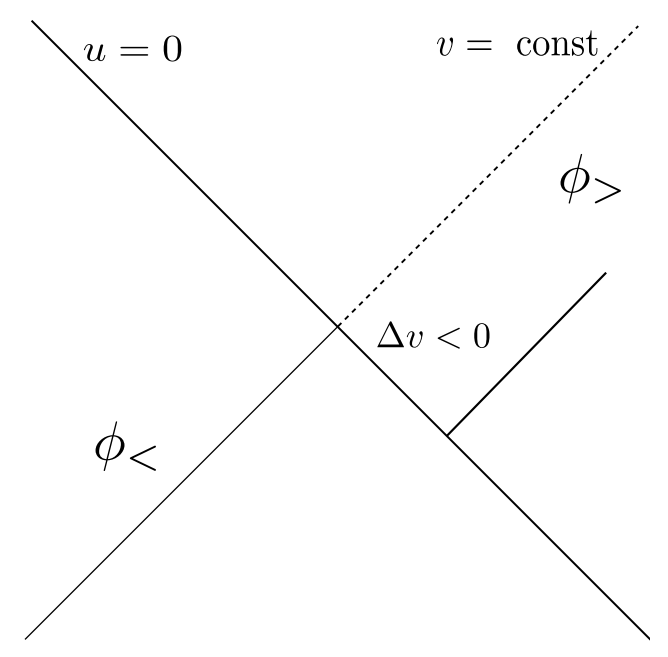

Figure 3.1: The shock wave profile corresponds to the line $u=0$. The graviton correponds to the solid line $v=$ const. After colliding with the shock wave, the graviton may return in a position such that $\Delta v<0$, outside its own light-cone and thus violating causality.

\footnotetext{
${ }^{2}$ Note that the condition $\mathcal{N}_{2} \geq 0$ does not fully guarantee that causality is preserved. More tighter constraints may still arise if we change the shock wave profile; see for instance [81].
} 


\section{Chapter 4}

\section{Chern-Simons diffusion rate from higher curvature gravity}

An important transport coefficient in the study of non-Abelian plasmas is the ChernSimons diffusion rate, which parameterizes the rate of transition among the degenerate vacua of a gauge theory. We compute this quantity at strong coupling, via holography, using two theories of gravity with higher curvature corrections, namely Gauss-Bonnet gravity and quasi-topological gravity. We find that these corrections may either increase or decrease the result obtained from Einstein's gravity, depending on the value of the couplings. The Chern-Simons diffusion rate for Gauss-Bonnet gravity decreases as the shear viscosity over entropy ratio is increased.

\subsection{Chern-Simons diffusion rate}

Non-Abelian gauge theories enjoy a rich topological structure, as displayed for example by the presence of infinitely many degenerate vacuum states. Transitions among these vacua are possible through quantum tunneling or thermal jumps and are parameterized by the change in the Chern-Simons number $N_{\mathrm{CS}}$, the topological invariant that classifies the different vacua:

$$
\Delta N_{\mathrm{CS}}=\frac{g_{\mathrm{YM}}^{2}}{8 \pi^{2}} \int d^{4} x \operatorname{tr} F \wedge F .
$$

Gauge field configurations responsible for a non-vanishing $\Delta N_{\mathrm{CS}}$ are either instantons, which are suppressed in the coupling constant both at zero and finite temperature, or, at finite temperature, thermal solutions called sphalerons [82-85], which are not necessarily suppressed. The rate of change of $N_{\mathrm{CS}}$ per unit volume $V$ and unit time $t$ is a transport 
coefficient called the Chern-Simons diffusion rate, $\Gamma_{\mathrm{CS}}$, which is defined as

$$
\Gamma_{\mathrm{CS}} \equiv \frac{\left\langle\Delta N_{\mathrm{CS}}^{2}\right\rangle}{V \cdot t}=\left(\frac{g_{\mathrm{YM}}^{2}}{8 \pi^{2}}\right)^{2} \int d^{4} x\langle\mathcal{O}(x) \mathcal{O}(0)\rangle, \quad \mathcal{O}(x)=(\operatorname{tr} F \wedge F)(x) .
$$

The Chern-Simons diffusion rate is important in electroweak baryogenesis and in the study of a wealth of CP-odd processes, as for example the chiral magnetic effect in QCD [86, 87]. A non-vanishing $\Gamma_{\mathrm{CS}}$ indicates a chiral asymmetry and the subsequent formation of domains with a non-zero net chirality. It has been computed at weak coupling for a $S U\left(N_{\mathrm{c}}\right)$ Yang-Mills theory and its parametric behavior has been found to be [88-91]

$$
\Gamma_{\mathrm{CS}}^{\mathrm{weak}} \propto \lambda^{5} \log \left(\frac{1}{\lambda}\right) T^{4}, \quad \lambda \ll 1,
$$

where $\lambda \equiv g_{\mathrm{YM}}^{2} N_{\mathrm{c}}$ is the 't Hooft coupling and $T$ is the temperature. Motivated by the strongly coupled nature of the quark-gluon plasma (QGP) produced in relativistic heavy ion collisions, this quantity has also been computed at strong coupling via holography in Einstein's gravity, with the result [75]

$$
\Gamma_{\mathrm{CS}}^{\text {Einstein }}=\frac{\lambda^{2}}{256 \pi^{3}} T^{4}, \quad N_{\mathrm{c}} \gg 1 \text { and } \lambda \gg 1
$$

Other holographic studies of $\Gamma_{\mathrm{CS}}$ include [92-95].

It is interesting to understand modifications to eq. (4.4) due to higher curvature corrections. These are in principle dictated by string theory and would correspond, in the gauge theory, to corrections in $1 / N_{\mathrm{c}}$ and $1 / \lambda$. In this note, we limit our attention to two specific types of higher curvature extensions of Einstein's gravity and compute the ChernSimons diffusion rate in Gauss-Bonnet (GB) gravity [8-11] ${ }^{1}$ and in quasi-topological (QT) gravity [96].

These theories contain higher derivative terms, but are such that the equations of motion for the metric are still second order, ${ }^{2}$ thus avoiding pathologies. It is not yet clear whether they emerge as a low energy solution of some string theory, so that their ultimate relevance is not yet established, but they do present very nice features. Besides being free of pathologies, as mentioned already, they possess a large class of black hole solutions and admit AdS boundary conditions, motivating their use in a 'bottom-up' approach to the study of strongly coupled plasmas.

Various physical observables relevant in the study of the QGP have already been computed from these theories. Notable examples are given by [97] and [98], where the

\footnotetext{
${ }^{1}$ For reviews of Gauss-Bonnet and, more generally, Lovelock gravity in the context of the AdS/CFT correspondence see e.g. $[13,14]$. A nice overview of black hole solutions can be found in [17].

${ }^{2}$ For quasi-topological gravity this is true for the linearized equations in an $A d S_{5}$ background.
} 
shear viscosity to entropy ratio was studied. There it was found that higher derivative terms may violate the famous bound $\eta / s \geq 1 / 4 \pi$ proposed in [76].

\subsection{Gravity setup and results}

We consider gravity in 5-dimensions with a negative cosmological constant and the inclusion of the GB and QT terms, with action given by

$$
S=\frac{1}{16 \pi G_{5}} \int d^{5} x \sqrt{-g}\left[R+\frac{12}{L^{2}}+\frac{L^{2}}{2} \lambda_{\mathrm{GB}} \mathcal{L}_{2}+L^{4} \mu \Xi_{3}\right]+S_{\mathrm{bdry}} .
$$

Here $L$ is a length scale, later to be related with the AdS radius, $\lambda_{\mathrm{GB}}$ and $\mu$ are two dimensionless couplings, the quadratic term $\mathcal{L}_{2}=R^{2}-4 R_{m n} R^{m n}+R_{m n r s} R^{\text {mnrs }}$ is the Euler density of GB gravity, and $\Xi_{3}$ is the cubic term of QT gravity, whose explicit expression [96] won't be needed in the following. $S_{\mathrm{bdry}}$ is a boundary term that makes the variational problem well posed. Remarkably, this action admits ${ }^{3}$ planar AdS black hole solutions, given by $[8,96]$

$$
d s^{2}=\frac{L^{2}}{z^{2}}\left(-a^{2} f(z) d t^{2}+\frac{d z^{2}}{f(z)}+\sum_{i=1}^{3} d x_{i}^{2}\right)
$$

where $x^{\mu}=\left(t, x_{i}\right)$ are the gauge theory coordinates, $z$ is the radial AdS coordinate, $a$ is a constant, and $f(z)$ is a function that vanishes at the horizon, $z=z_{\mathrm{H}}$, and which will be given below. The AdS boundary is located at $z=0$. Requiring $c=1$ in the boundary theory fixes $a=f(0)^{-1 / 2}$. The black hole temperature is given by $T=a / \pi z_{\mathrm{H}}$.

In the AdS/CFT correspondence, the operator $\mathcal{O}(x)$ of eq. (4.2) is coupled to a bulk scalar field, $\chi\left(z, x^{\mu}\right)$, whose background value is zero in the present case. The (retarded) 2-point function of $\mathcal{O}(x)$ can be obtained by computing the fluctuations of this field, $\delta \chi\left(z, x^{\mu}\right)$, subject to infalling boundary conditions at the horizon and plugging the result into the corresponding boundary action, minimally coupled to eq. (4.5). This procedure is detailed in [75], where, as a first step, the definition (4.2) is rewritten in Fourier space as

$$
\Gamma_{\mathrm{CS}}=-\left(\frac{g_{\mathrm{YM}}^{2}}{8 \pi^{2}}\right)^{2} \lim _{\omega \rightarrow 0} \frac{2 T}{\omega} \operatorname{Im} G^{\mathrm{R}}(\omega, \mathbf{0}) .
$$

$G^{\mathrm{R}}(\omega, \mathbf{0})$ is the retarded Green's function associated to $\mathcal{O}(x)$, evaluated at zero spatial momentum. It can be calculated as

$$
G^{\mathrm{R}}(\omega, \mathbf{0})=\left.\frac{N_{\mathrm{c}}^{2}}{8 \pi^{2} L^{3}} \sqrt{-g} g^{z z} f_{-k}(z) \partial_{z} f_{k}(z)\right|_{z \rightarrow 0},
$$

\footnotetext{
${ }^{3}$ This is true for appropriate values of the couplings. For example, it must be $\lambda_{\mathrm{GB}}<\frac{1}{4}$.
} 
where $f_{k}(z)$ is the Fourier mode of the scalar field fluctuation

$$
\delta \chi\left(z, x^{\mu}\right)=\int \frac{d^{4} k}{(2 \pi)^{4}} e^{i k \cdot x} f_{k}(z)
$$

which can be obtained as a solution of the equation

$$
\frac{1}{\sqrt{-g}} \partial_{z}\left(\sqrt{-g} g^{z z} \partial_{z} f_{k}(z)\right)-g^{\mu \nu} k_{\mu} k_{\nu} f_{k}(z)=0, \quad k_{\mu}=(-\omega, \mathbf{k}) \text {. }
$$

It is convenient to work with the dimensionless coordinate $u$ defined as $u=z^{2} / z_{\mathrm{H}}^{2}$, in terms of which we have (setting already $\mathbf{k}=0$ )

$$
\partial_{u}^{2} f_{k}(u)+\left[\partial_{u} \ln \frac{f(u)}{u}\right] \partial_{u} f_{k}(u)+\frac{\mathrm{w}^{2}}{u f(u)^{2}} f_{k}(u)=0,
$$

where we have defined for convenience the dimensionless frequency $\mathrm{w} \equiv \omega / 2 \pi T$.

The 'blackening factor' $f(u)$ is defined implicitly through the cubic equation [96]

$$
1-f(u)+\lambda_{\mathrm{GB}} f(u)^{2}+\mu f(u)^{3}=u^{2} .
$$

Out of the three solutions, we select the one which is regular when $\mu \rightarrow 0$ and reproduces the expression $f(u)=\left(1-\sqrt{1-4 \lambda_{\mathrm{GB}}\left(1-u^{2}\right)}\right) / 2 \lambda_{\mathrm{GB}}$ of the GB case [15,17]. ${ }^{4}$ We recall that the couplings $\lambda_{\mathrm{GB}}$ and $\mu$ are constrained by requirements of unitarity, causality, and positivity of energy fluxes in the dual conformal field theory. It turns out that it must be $[98]^{5}$

$$
-0.36 \lesssim \lambda_{\mathrm{GB}} \lesssim 0.12, \quad|\mu| \lesssim 0.001
$$

In view of this, we will solve eqs. (4.11) and (4.12) exactly in $\lambda_{\mathrm{GB}}$, but only approximately to first order in small $\mu$. This allows us to we write explicitly

$$
\begin{aligned}
f(u)= & \frac{1}{2 \lambda_{\mathrm{GB}}}\left(1-\sqrt{1-4 \lambda_{\mathrm{GB}}\left(1-u^{2}\right)}\right)+ \\
& +\frac{1-\sqrt{1-4 \lambda_{\mathrm{GB}}\left(1-u^{2}\right)}-\lambda_{\mathrm{GB}}\left(1-u^{2}\right)\left(3-\sqrt{1-4 \lambda_{\mathrm{GB}}\left(1-u^{2}\right)}\right)}{2 \lambda_{\mathrm{GB}}^{3} \sqrt{1-4 \lambda_{\mathrm{GB}}\left(1-u^{2}\right)}} \mu+O\left(\mu^{2}\right) .
\end{aligned}
$$

There is no known analytic solution to eq. (4.11), but this is not needed anyway,

\footnotetext{
${ }^{4}$ The GB case has also another solution for $f(u)$, with a plus sign in front of the square root, which is however known to be unstable and to contain ghosts.

${ }^{5}$ The constraints on $\lambda_{\mathrm{GB}}$ and $\mu$ are not independent; see Fig. 1 of [98]. In particular, in the case of pure GB gravity $(\mu=0)$, the allowed range of $\lambda_{\mathrm{GB}}$ is $-7 / 36 \leq \lambda_{\mathrm{GB}} \leq 9 / 100$. For $\mu<0$ there are instabilities in the graviton tensor channel for momenta above a certain critical value [98]. Since $\Gamma_{\mathrm{CS}}$ is computed at $\mathbf{k}=0$ we do not worry about this here.
} 
since only the small frequency behavior $\mathrm{w} \rightarrow 0$ of the Green's function enters in the Chern-Simons diffusion rate. We can then make the following Ansatz:

$$
f_{k}(u)=f(u)^{-i \frac{\mathrm{w}}{2}}\left(F_{0}(u)+\mathrm{w}\left(F_{1}^{(0)}(u)+\mu F_{1}^{(1)}(u)+O\left(\mu^{2}\right)\right)+O\left(\mathrm{w}^{2}\right)\right)
$$

Here $F_{0}, F_{1}^{(0)}$, and $F_{1}^{(1)}$ are regular functions at the horizon, $u=1$. In fact, we can choose them to be such that

$$
F_{0}(1)=1, \quad F_{1}^{(0)}(1)=\frac{i}{2} \log 2, \quad F_{1}^{(1)}(1)=0 .
$$

The exponent of $f(u)$ has been chosen to give infalling boundary conditions at the horizon, which correspond to having a retarded Green's function in the boundary. Expanding around $u=1$, one finds in fact that $f_{k}(u) \sim(1-u)^{-i \frac{\mathrm{w}}{2}}\left(1+O\left(\mathrm{w}^{2}\right)\right)$. Plugging the Ansatz above in eq. (4.11), it is easy ${ }^{6}$ to find the following solutions which respect the boundary conditions above:

$$
\begin{aligned}
& F_{0}(u)=1, \quad F_{1}^{(0)}(u)=\frac{i}{2}\left(1+\log 2-\sqrt{1-4 \lambda_{\mathrm{GB}}\left(1-u^{2}\right)}\right) \\
& F_{1}^{(1)}(u)=-\frac{i}{8 \lambda_{\mathrm{GB}}^{2}} \frac{1-2 \lambda_{\mathrm{GB}}\left(1-u^{2}\right)-8 \lambda_{\mathrm{GB}}^{2}\left(1-u^{2}\right)^{2}-\sqrt{1-4 \lambda_{\mathrm{GB}}\left(1-u^{2}\right)}}{1-4 \lambda_{\mathrm{GB}}\left(1-u^{2}\right)}
\end{aligned}
$$

Using eqs. (4.7) and (4.8), and keeping only terms linear in $\mu$, we finally arrive at

$$
\Gamma_{\mathrm{CS}}=\Gamma_{\mathrm{CS}}^{\mathrm{Einstein}}\left(H^{(0)}\left(\lambda_{\mathrm{GB}}\right)+\mu H^{(1)}\left(\lambda_{\mathrm{GB}}\right)+O\left(\mu^{2}\right)\right)
$$

with

$$
\begin{aligned}
& H^{(0)}\left(\lambda_{\mathrm{GB}}\right)=\left(\frac{1-\sqrt{1-4 \lambda_{\mathrm{GB}}}}{2 \lambda_{\mathrm{GB}}}\right)^{3 / 2} \\
& H^{(1)}\left(\lambda_{\mathrm{GB}}\right)=\frac{3}{4} \sqrt{\frac{1-\sqrt{1-4 \lambda_{\mathrm{GB}}}}{2 \lambda_{\mathrm{GB}}^{7}\left(1-4 \lambda_{\mathrm{GB}}\right)}}\left(1-\sqrt{1-4 \lambda_{\mathrm{GB}}}-\lambda_{\mathrm{GB}}\left(3-\sqrt{1-4 \lambda_{\mathrm{GB}}}\right)\right)
\end{aligned}
$$

We stress that this result is fully non-perturbative in $\lambda_{\mathrm{GB}}$, at any order in $\mu$. We see that the Chern-Simons diffusion rate in GB and QT gravity is a rescaling of the result in eq. (4.4). The dependence on $T$ is dictated by conformal invariance: $\Gamma_{\mathrm{CS}}$ must be proportional to $T^{4}$ for dimensional reasons, with the factor of proportionality depending solely on the dimensionless parameters, which are $\lambda_{\mathrm{GB}}$ and $\mu .^{7}$ Fig. 4.1(Left) shows the two terms in $\Gamma_{\mathrm{CS}}$ as functions of $\lambda_{\mathrm{GB}}$. Both terms are finite, monotonically increasing and positive in

\footnotetext{
${ }^{6}$ The equations simplify if one changes coordinates $u \rightarrow \sqrt{1-4 \lambda_{\mathrm{GB}}\left(1-u^{2}\right)}$ in intermediate steps.

${ }^{7}$ An interesting context where this does not happen is Improved Holographic QCD [95], where the absence of conformal symmetry makes $\Gamma_{\mathrm{CS}} / \Gamma_{\mathrm{CS}}^{\text {Einstein }}$ depend on $T$.
} 

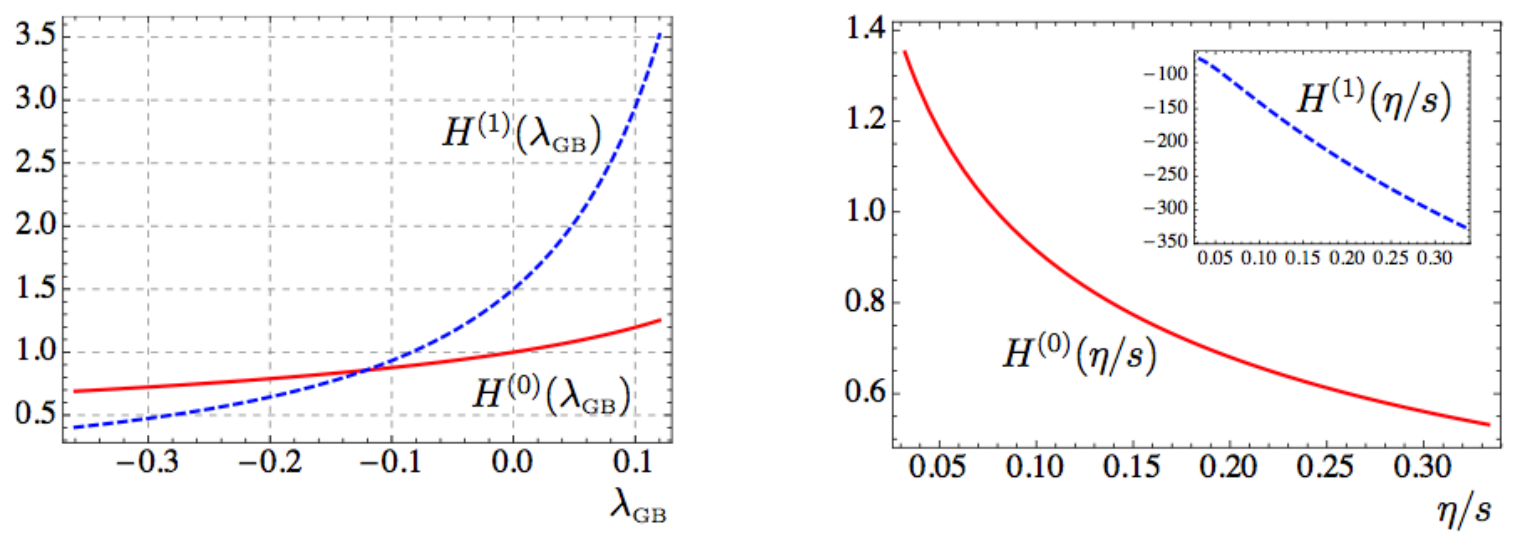

Figure 4.1: (Left) The factors $H^{(0)}\left(\lambda_{G B}\right)$ (red, solid curve) and $H^{(1)}\left(\lambda_{G B}\right)$ (blue, dashed curve) as functions of $\lambda_{G B}$. (Right) The same factors as functions of $\eta / s$. The plots are exact in $\lambda_{G B}$ and in $\eta / s$, whose allowed ranges are obtained from eqs. (4.13) and (4.20). In these ranges, the corrections to eq. (4.4) are finite and cannot make the diffusion rate arbitrarily small.

the allowed range of $\lambda_{\mathrm{GB}}$, given in eq. (4.13). The GB contribution can be either smaller or larger than 1, depending on the sign of $\lambda_{\mathrm{GB}}$, and the corresponding Chern-Simons diffusion rate can be either smaller or larger than the result obtained from Einstein's gravity, but, in the allowed range of eq. (4.13), cannot get arbitrarily small.

Fig. 4.1(Right) displays the two contributions $H^{(0)}$ and $H^{(1)}$ as functions of the shear viscosity over entropy ratio, which is given by $[97,98]$

$$
\frac{\eta}{s}=\frac{1}{4 \pi}\left[1-4 \lambda_{\mathrm{GB}}-36 \mu\left(9-64 \lambda_{\mathrm{GB}}+128 \lambda_{\mathrm{GB}}^{2}\right)\right]+O\left(\mu^{2}\right) .
$$

We observe that $\Gamma_{\mathrm{CS}}$ for GB gravity decreases as $\eta / s$ is increased (for QT gravity this depends on the sign of $\mu$, whose contribution is however suppressed). It would be very interesting to understand if there is a microscopic interpretation of this behavior.

\subsection{Discussion}

Understanding corrections away from the infinite $N_{\mathrm{c}}$ and infinite $\lambda$ limit is clearly of the utmost importance in order to make contact with realistic systems. Unfortunately, loop and stringy corrections are in general hard to compute, so that our philosophy in this note has been to consider two simple extensions of Einstein's gravity with higher curvature terms, just to gain a qualitative understanding of how such terms might modify the computation of an important observable in strongly coupled non-Abelian plasmas. ${ }^{8}$

\footnotetext{
${ }^{8}$ Besides making things more realistic, the study of how higher derivative terms affect the computation of gauge theory observables might also be useful to put constraints on the string landscape, e.g. by
} 
This is similar in spirit to what has been done, in [97] for GB gravity and in [98] for QT gravity, for the shear viscosity over entropy ratio, which turned out to be lower in these theories than what it is in Einstein's gravity. In [97] it was in fact found to be $\eta / s=\left(1-4 \lambda_{\mathrm{GB}}\right) / 4 \pi$ and in [98] to be $\eta / s \gtrsim 0.4140 / 4 \pi$, both cases in violation of the bound proposed in [76]. ${ }^{9}$ It is interesting to observe that a subsequent computation [105] in a setting [106-108] where $\alpha^{\prime}$-corrections can be solved exactly yielded the same qualitative behavior, with the bound $\eta / s \geq 1 / 4 \pi$ being violated.

The presence of the new gravitational couplings $\lambda_{\mathrm{GB}}$ and $\mu$ corresponds on the boundary to considering conformal field theories which are more generic than the ones usually studied. In particular, a non-vanishing $\lambda_{\mathrm{GB}}$ results in having independent central charges $a \neq c[19,20]$, whereas a non-vanishing $\mu$ also results in the breaking of supersymmetry [96]. For these reasons, these theories, even if they turn out to be just toy models without a UV completion, may still be useful in exploring situations which require an understanding of holography in non-trivial backgrounds.

excluding ranges of parameters that would produce pathologies in the dual gauge theory, as suggested in $[16]$.

${ }^{9}$ See also, for instance, [99-103] and [22,29] for violations of the bound in an anisotropic plasma. A status report of the Kovtun-Son-Starinets conjecture can be found in [104]. 


\section{Chapter 5}

\section{Anisotropic black branes in higher curvature gravity}

The AdS/CFT correspondence has been recently applied to the study of the quark gluon plasma (QGP) produced in heavy ion collisions, motivated by the behavior of the plasma as a strongly coupling system that makes the application of traditional perturbation theory techniques inviable. It is clear that to study the real-world QGP one would need the holographic dual to QCD, which is not currently known. Finite temperature $\mathcal{N}=4$ SYM has given us good insight on the qualitative features of the strong coupling dynamics of the plasma. Deform the $\mathcal{N}=4 \mathrm{SYM}$ to incorporate features present in the real-world QGP is an obvious step towards a more reliable holographic description of the QGP. For example, the QGP produced in heavy ion collisions is initially anisotropic due to the preferred direction of the beam collision.

An anisotropic black hole solution in type IIB supergravity that can be used to describe an anisotropic strongly coupled plasma was recently discovered in [22]. This solution presents an anisotropic scaling of the coordinates in the interior of the space sourced by an axion field, corresponding on the gauge theory side to a position-dependent theta-angle term deforming the $\mathcal{N}=4 \mathrm{SYM}$ theory. The idea of this work is to find the equivalent of the solution of [22] including the first Lovelock correction, motivated by the fact that higher curvature terms appear as $\alpha^{\prime}$ corrections to supergravity, corresponding in the gauge theory side, to finite 't Hooft coupling constant.

This chapter is organized as follows. In Sec. 5.1 we present our solution and compute its temperature and entropy density. In Sec. 5.2 we carry out the holographic renormalization, obtaining general formulas for the expectation values of the stress tensor and of the axion and dilaton operators. In Sec. 5.3 we specialize those formulas to the case at hand and discuss the various features of energy density and pressures. We finally discuss our results in Sec. 5.4. The explicit expressions of the solution can be found in Appendix B. 


\subsection{Action and solution}

We are interested in five-dimensional gravity with a negative cosmological constant and the inclusion of a Gauss-Bonnet term, which we also couple to an axion-dilaton system in the following way

$$
S=\frac{1}{16 \pi G} \int d^{5} x \sqrt{-g}\left[R+\frac{12}{\ell^{2}}-\frac{1}{2}(\partial \phi)^{2}-\frac{e^{2 \phi}}{2}(\partial \chi)^{2}+\frac{\ell^{2}}{2} \lambda_{\mathrm{GB}} \mathcal{L}_{\mathrm{GB}}\right]+S_{\mathrm{GH}} .
$$

The scalar fields $\phi$ and $\chi$ are the dilaton and axion, respectively, $\lambda_{\mathrm{GB}}$ is the (dimensionless) Gauss-Bonnet coupling and

$$
\mathcal{L}_{\mathrm{GB}}=R^{2}-4 R_{m n} R^{m n}+R_{m n r s} R^{m n r s}
$$

is the Gauss-Bonnet term. $\ell$ is a parameter with dimensions of length that we set to one in what follows, without loss of generality. We use the Latin indices $m, n, \ldots$ for the fivedimensional coordinates $(t, x, y, z, u)$, with $u$ being the radial coordinate. The term $S_{\mathrm{GH}}$ is the usual Gibbons-Hawking term, necessary to render the variational problem well posed. When $\lambda_{\mathrm{GB}}=0$ the action above can be obtained from type IIB superstrings [22,23], but this is no longer true when the Gauss-Bonnet coupling is turned on. In fact, it is not clear whether (5.1) can be obtained from any string theory compactification, so that our point of view in the present work is 'bottom-up'.

The field equations for the metric resulting from the action above are given by

$$
R_{m n}-\frac{1}{2} g_{m n} R+\frac{\lambda_{\mathrm{GB}}}{2} \delta \mathcal{L}_{\mathrm{GB} m n}=\frac{1}{2} \partial_{m} \phi \partial_{n} \phi+\frac{1}{2} e^{2 \phi} \partial_{m} \chi \partial_{n} \chi-\frac{g_{m n}}{4}\left[(\partial \phi)^{2}+e^{2 \phi}(\partial \chi)^{2}-12\right],
$$

where

$$
\delta \mathcal{L}_{\mathrm{GB} m n}=-\frac{g_{m n}}{2} \mathcal{L}_{\mathrm{GB}}-4 R_{m}{ }^{r} R_{r n}+2 R_{m n} R-4 R^{r s} R_{m r n s}+2 R_{m}{ }^{r s t} R_{n r s t}
$$

is the variation of the Gauss-Bonnet term. The equations for the dilaton and axion read instead

$$
\partial_{m}\left(\sqrt{-g} g^{m n} \partial_{n} \phi\right)=\sqrt{-g} e^{2 \phi}(\partial \chi)^{2}, \quad \partial_{m}\left(\sqrt{-g} e^{2 \phi} g^{m n} \partial_{n} \chi\right)=0
$$

We want to obtain a solution which displays a spatial anisotropy. This is achieved by singling out one direction, say the $z$-direction, which will be later identified with the 'beam direction' in a heavy ion collision experiment occurring in the boundary theory. To get an anisotropy between the $z$-direction and the $x y$-directions (the transverse plane to 
the beam), we consider the following Ansatz ${ }^{1}$

$$
d s^{2}=\frac{1}{u^{2}}\left(-F B d t^{2}+d x^{2}+d y^{2}+H d z^{2}+\frac{d u^{2}}{F}\right) .
$$

All the metric components $F, B$, and $H$, as well as the dilaton $\phi$, depend solely on the radial coordinate $u$. This guarantees that the solution be static. In this parametrization the boundary is located at $u=0 . F$ is a 'blackening factor' that introduces an horizon in the geometry at $u=u_{\mathrm{H}}$, where $F\left(u_{\mathrm{H}}\right)=0$. There is a scaling symmetry in the coordinates $t$ and $z$ that allows us to set $B_{\mathrm{bdry}} F_{\mathrm{bdry}}=H_{\mathrm{bdry}}=1$, thus recovering a canonically normalized AdS metric in the UV region (with radius $1 / \sqrt{F_{\text {bdry }}}$ ). Here and in what follows we use the subscript 'bdry' to denote the value of the fields at $u=0$.

Following $[21,22]$ we consider an axion field which has a constant profile in the radial direction and depends linearly on $z$

$$
\chi=a z .
$$

The parameter $a$ has dimensions of energy and controls the amount of anisotropy. It is clear that this is a solution of the axion equation, since the metric is diagonal and the metric and dilaton do not depend on $z$.

In this work we limit ourselves to considering the case of small anisotropy, which will allow for an analytic solution of the equations of motion. To do this we expand all the fields around the (isotropic) Gauss-Bonnet black brane solution ${ }^{2}$

$$
\begin{aligned}
\phi(u) & =a^{2} \phi_{2}(u)+O\left(a^{4}\right), \\
F(u) & =F_{0}(u)+a^{2} F_{2}(u)+O\left(a^{4}\right), \\
B(u) & =B_{0}\left(1+a^{2} B_{2}(u)+O\left(a^{4}\right)\right), \\
H(u) & =1+a^{2} H_{2}(u)+O\left(a^{4}\right),
\end{aligned}
$$

where

$$
F_{0}(u)=\frac{1}{2 \lambda_{\mathrm{GB}}}\left(1-\sqrt{1-4 \lambda_{\mathrm{GB}}\left(1-\frac{u^{4}}{u_{\mathrm{H}}^{4}}\right)}\right), \quad \lambda_{\mathrm{GB}}<\frac{1}{4} .
$$

This is a solution of the equations of motion when $a=0$. In order to have a unit speed of light at the boundary we set

$$
B_{0}=\frac{1}{2}\left(1+\sqrt{1-4 \lambda_{\mathrm{GB}}}\right) .
$$

\footnotetext{
${ }^{1}$ Note that this Ansatz is slightly different than the one used in [22, 23].

${ }^{2}$ See e.g. [97] or [13] for a review.
} 
This is possible due to the scaling symmetry in $t$, which we have mentioned above. Note that only even powers of $a$ can appear in the expansion because of the symmetry $z \rightarrow-z$.

Luckily it is possible to solve the equations analytically at order $O\left(a^{2}\right)$. The equations at this order and the explicit solutions are detailed in App. B. A plot of representative solutions is contained in Figure 5.1, where the regularity of the geometry is explicitly exhibited.
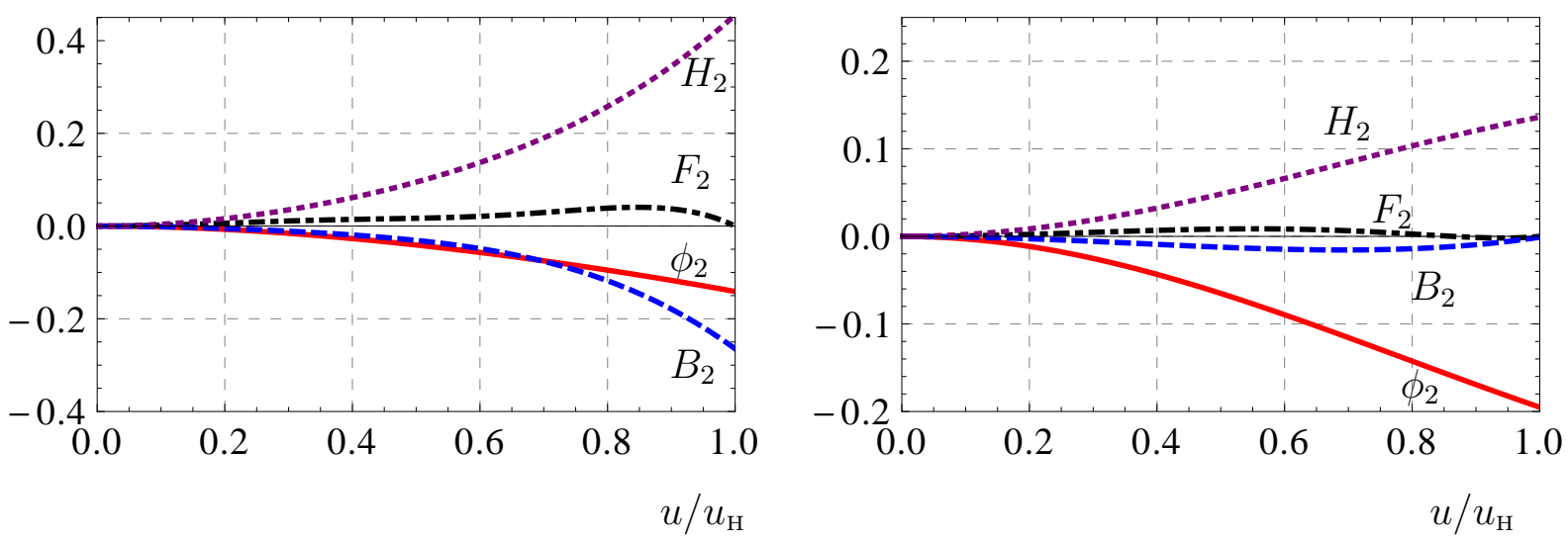

Figure 5.1: The metric functions at order $O\left(a^{2}\right)$ for $\lambda_{G B}=0.2$ (left) and $\lambda_{G B}=-0.2$ (right).

Here we just mention that we have fixed the integration constants in such a way that all the metric functions are regular at the horizon and moreover

$$
\phi_{2, \mathrm{bdry}}=F_{2, \mathrm{bdry}}=B_{2, \mathrm{bdry}}=H_{2, \mathrm{bdry}}=0,
$$

thus recovering AdS in the UV. A direct computation of the Kretschmann invariant $R_{m n p q} R^{m n p q}$ shows no singularity in the geometry except for $\lambda_{\mathrm{GB}}=1 / 4$, which is however excluded, as can be seen from (5.9).

Unfortunately, we have not been able to find analytic solutions beyond order $O\left(a^{2}\right)$ and most likely a numerical analysis will turn out to be necessary to go to higher anisotropies. This is however beyond the scope of the present work. It should be possible, in principle, to consider arbitrarily large values of $a$, as in the pure Einstein-Hilbert case of $[22,23]$.

The temperature of the solution can be computed as usual from the standard requirement that the (Euclideanized) metric be regular at $u=u_{\mathrm{H}}$. One finds that

$$
T=-\left.\frac{F^{\prime}(u) \sqrt{B(u)}}{4 \pi}\right|_{u=u_{\mathrm{H}}}
$$


Specializing to our solution this becomes ${ }^{3}$

$T=\sqrt{B_{0}}\left(\frac{1}{\pi u_{\mathrm{H}}}-\frac{2 B_{0}-6 \lambda_{\mathrm{GB}}+\sqrt{\lambda_{\mathrm{GB}}} \log \left(\frac{1+2 \sqrt{\lambda_{\mathrm{GB}}}}{1-2 \sqrt{\lambda_{\mathrm{GB}}}}\right)-\log \left(\frac{4 B_{0}}{\sqrt{1-4 \lambda_{\mathrm{GB}}}}\right)}{48 \pi\left(1-4 \lambda_{\mathrm{GB}}\right)} u_{\mathrm{H}} a^{2}+O\left(a^{4}\right)\right)$.

This equation can be easily inverted to find $u_{\mathrm{H}}$ as a function of $T$.

For planar black holes in GB gravity the entropy density is still given by the usual formula in terms of the area of the horizon. We find (here $V_{3}$ is the infinite volume $\left.\int d x d y d z\right)$

$$
s=\frac{A_{\mathrm{hor}}}{4 G V_{3}}=\frac{\pi}{4 G B_{0}^{3 / 2}}\left(\pi^{2} T^{3}+\frac{1}{8} T B_{0} a^{2}+O\left(a^{4}\right)\right)
$$

We notice that for $\lambda_{\mathrm{GB}}=0$ this matches the result obtained in $[22,23]$.

A final comment on the IR behavior of the geometry is in order. The solution of $[22,23]$ was interpolating between AdS boundary conditions in the UV and a Lifshitz-like scaling solution [21] in the IR. We believe that the finite $\lambda_{\mathrm{GB}}$ generalization discussed here does not share this feature with [22,23], although we have not been able to prove this rigorously. More specifically, we have not been able to find a scaling solution in the IR (even for $T=0$ ), as done in [21] for the case $\lambda_{\mathrm{GB}}=0$. One obstruction might be that Lifshitz solutions in GB gravity seem to require to tune the cosmological constant in ways that are not compatible with our equations. For example, in the case of GB gravity coupled to a massive vector field the condition for a Lifshitz scaling is that the cosmological constant be half of the usual value [109]. ${ }^{4}$ It would certainly be interesting to settle this point, but this goes beyond the scope of this work.

\subsection{Holographic renormalization}

In this section we use holographic renormalization techniques to compute the 1-point function of the boundary stress tensor associated to our gravitational system; see [111] for a review. In fact, we consider a generalization of (5.1), where the coefficient of the axion kinetic term is allowed to be a generic function $Z(\phi)$ of the dilaton. We also maintain the metric and axion-dilaton generic. We use the recursive Hamilton-Jacobi method that was introduced in [24] for axion-dilaton gravity without Gauss-Bonnet term. Our main result are the expressions (5.57)-(5.60) for $\left\langle T_{i j}\right\rangle,\left\langle\mathcal{O}_{\phi}\right\rangle$ and $\left\langle\mathcal{O}_{\chi}\right\rangle$, which are general and which

\footnotetext{
${ }^{3}$ Note that this expression is valid, and real, even for negative $\lambda_{\mathrm{GB}}$.

${ }^{4} \mathrm{~A}$ flow between a Lifshitz solution in the UV and an AdS solution in the IR for GB-gravity coupled to a massive vector field was discussed in [110].
} 
we specialize to our solution (5.8) in the next section.

\subsubsection{Radial evolution Hamiltonian}

The recursive Hamilton-Jacobi method of [24], which we follow closely in this section, makes use of the ADM formalism, in which a manifold $\mathcal{M}$ is foliated with hypersurfaces $\Sigma_{r}$ of constant radial coordinate, which we call $r$ in this section. In this coordinate, which plays the role of Hamiltonian time, the boundary is located at $r=\infty$. The metric on $\mathcal{M}$ takes the form

$$
d s^{2}=\left(N^{2}+N_{i} N^{i}\right) d r^{2}+2 N_{i} d r d x^{i}+\gamma_{i j} d x^{i} d x^{j}
$$

where $N$ and $N_{i}$ are the lapse and shift function, respectively, and $\gamma_{i j}$ is the induced metric on $\Sigma_{r}$. We use the Latin indices $i, j, \ldots$ to label the coordinates $(t, x, y, z)$ on $\Sigma_{r}$. In terms of these fields, the extrinsic curvature is given by

$$
K_{i j}=\frac{1}{2 N}\left(\dot{\gamma}_{i j}-D_{i} N_{j}-D_{j} N_{i}\right)
$$

with the dot denoting differentiation with respect to $r$ and $D_{i}$ being the covariant derivative associated to $\gamma_{i j}$.

The axion-dilaton part of (5.1), without Gauss-Bonnet contribution, turns out to be given by $^{5}$

$$
\begin{aligned}
S_{\text {axion-dilaton }}=\int_{\mathcal{M}} d^{d} x \sqrt{-g}[ & \mathcal{R}+K^{2}-K_{i j}^{2}+(d-1)(d-2) \\
& \left.-\frac{1}{2}\left(\dot{\phi}^{2}+Z(\phi) \dot{\chi}^{2}+\gamma^{i j}\left(\partial_{i} \phi \partial_{j} \phi+Z(\phi) \partial_{i} \chi \partial_{j} \chi\right)\right)\right](.5 .1
\end{aligned}
$$

Here and in the following we leave the function in the axion kinetic term as a generic function of the dilaton, $Z(\phi)$. Later we will specialize to $Z(\phi)=e^{2 \phi}$ and to $d=5$, which is the case considered in the previous section. We denote with the calligraphic fonts $\mathcal{R}$, $\mathcal{R}_{i j}$, etc. the curvature on $\mathcal{M}$ computed in terms of $N, N_{i}$ and $\gamma_{i j}$. All the contractions of the $i, j, \ldots$ indices are performed with $\gamma^{i j}$. The Gauss-Bonnet contribution is (see for example eq. (2.8) of [25])

$$
\begin{aligned}
& S_{\mathrm{GB}}=\frac{1}{2} \int_{\mathcal{M}} d^{d} x \sqrt{-g}\left[\left(\mathcal{R}^{2}+\right.\right.\left.K^{2}-K_{i j}^{2}\right)^{2}-4\left(\mathcal{R}_{i j}+K K_{i j}-K_{i k} K_{j}^{k}\right)^{2} \\
&+\left(\mathcal{R}_{i j k l}+K_{i k} K_{j l}-K_{i l} K_{j k}\right)^{2}-\frac{4}{3} K^{4}+8 K^{2} K_{i j}^{2} \\
&\left.\quad-\frac{32}{3} K K_{i}^{j} K_{j}^{k} K_{k}^{i}-4\left(K_{i j}^{2}\right)^{2}+8 K_{i}^{j} K_{j}^{k} K_{k}^{l} K_{l}^{i}\right]
\end{aligned}
$$

\footnotetext{
${ }^{5}$ We gauge-fix $N=1$ e $N_{i}=0$ and set, for this section, $\frac{1}{16 \pi G}=1$. Note that we use a different normalization (a factor of $1 / 2$ ) in our scalar kinetic terms compared to the scalar kinetic terms in [24].
} 
with $K=\gamma^{i j} K_{i j}$. The Gibbons-Hawking terms have already been included in the actions above, but they get canceled by boundary terms coming from the bulk actions. The total action is then

$$
S=S_{\text {axion-dilaton }}+\lambda_{\mathrm{GB}} S_{\mathrm{GB}}
$$

The next ingredient in the algorithm is to compute the Hamiltonian for radial evolution, which is associated to the Lagrangian $L$ defined by $S=\int d r L$. To this scope, we need the canonical conjugate momenta

$$
\begin{aligned}
& \pi^{i j} \equiv \frac{1}{\sqrt{-\gamma}} \frac{\delta L}{\delta \dot{\gamma}_{i j}} \\
& =\gamma^{i j} K-K^{i j}+\lambda_{\mathrm{GB}}\left[\gamma^{i j}\left(\mathcal{R} K-2 \mathcal{R}_{k l} K^{k l}\right)-\mathcal{R} K^{i j}-2 \mathcal{R}^{i j} K+4 \mathcal{R}^{k(i} K_{k}^{j)}\right. \\
& +2 \mathcal{R}^{i k j l} K_{k l}+\frac{1}{3} \gamma^{i j}\left(-K^{3}+3 K K_{k l}^{2}-2 K_{k}^{l} K_{l}^{m} K_{m}^{k}\right) \\
& \left.+K^{2} K^{i j}-2 K K_{k}^{i} K^{j k}-K^{i j} K_{k l}^{2}+2 K_{k}^{i} K_{l}^{k} K^{j l}\right], \\
& \pi_{\phi} \equiv \frac{1}{\sqrt{-\gamma}} \frac{\delta L}{\delta \dot{\phi}}=-\dot{\phi}, \quad \pi_{\chi} \equiv \frac{1}{\sqrt{-\gamma}} \frac{\delta L}{\delta \dot{\chi}}=-Z(\phi) \dot{\chi}
\end{aligned}
$$

In our solution it is clear that $\pi_{\chi}=0$, but we keep this term in this section for full generality. The Hamiltonian for radial evolution is then given by

$$
H=\int_{\Sigma_{r}} d^{d-1} x \sqrt{-\gamma}\left(2 \pi^{i j} K_{i j}+\pi_{\phi} \dot{\phi}+\pi_{\chi} \dot{\chi}\right)-L
$$

where we used that $K_{i j}=\dot{\gamma}_{i j} / 2$ in the chosen gauge. To write the Hamiltonian in terms of the canonical momenta and induced metric one needs to invert (5.20), which is a complicated system of nonlinear equations. This has been done in [25], but only to first order in $\lambda_{\mathrm{GB}}$. In this section we also limit ourselves to this regime, for simplicity (although, we repeat, our solution (5.8) is fully non-perturbative in $\lambda_{\mathrm{GB}}$ ).

Let us derive the expression for the Hamiltonian of radial evolution in (5.26). The starting point is

$$
H=\int_{\Sigma_{r}} d^{d-1} x \sqrt{-\gamma}\left(2 \pi^{i j} K_{i j}+\pi_{\phi} \dot{\phi}+\pi_{\chi} \dot{\chi}\right)-L
$$

where we write $L=\tilde{L}+L_{\text {axion-dilaton }}$, with

$$
L_{\text {axion-dilaton }}=-\frac{1}{2} \int_{\Sigma_{r}} d^{d-1} x \sqrt{-\gamma}\left[\dot{\phi}^{2}+Z(\phi) \dot{\chi}^{2}+\left(\partial_{i} \phi\right)^{2}+Z(\phi)\left(\partial_{i} \chi\right)^{2}\right] .
$$


We can then separate

$$
H=\underbrace{\left(\int_{\Sigma_{r}} d^{d-1} x \sqrt{-\gamma} 2 \pi^{i j} K_{i j}-\tilde{L}\right)}_{\equiv \tilde{H}}+\underbrace{\left(\int_{\Sigma_{r}} d^{d-1} x \sqrt{-\gamma}\left(\pi_{\phi} \dot{\phi}+\pi_{\chi} \dot{\chi}\right)-L_{\text {axion-dilaton }}\right)}_{\equiv H_{\text {axion-dilaton }}} .
$$

We note that $\tilde{H}$ is exactly the Hamiltonian in eq. (2.12) of [25] (up to an overall minus sign). For $H_{\text {axion-dilaton we have }}$

$$
H_{\text {axion-dilaton }}=-\int_{\Sigma_{r}} d^{d-1} x \sqrt{-\gamma}\left(\pi_{\phi}^{2}+\frac{\pi_{\chi}^{2}}{Z(\phi)}-\frac{1}{2}\left(\partial_{i} \phi\right)^{2}-\frac{1}{2} Z(\phi)\left(\partial_{i} \chi\right)^{2}\right) .
$$

Writing this in terms of the canonical momenta and induced metric leads to

$$
\begin{aligned}
& H=-\int_{\Sigma_{r}} d^{d-1} x \sqrt{-\gamma}\left[\mathcal{R}+(d-1)(d-2)-\frac{1}{d-2}\left(\pi_{i}^{i}\right)^{2}+\pi_{i j}^{2}+\pi_{\phi}^{2}+\frac{\pi_{\chi}^{2}}{Z(\phi)}\right. \\
& \quad-\frac{1}{2}\left(\left(\partial_{i} \phi\right)^{2}+Z(\phi)\left(\partial_{i} \chi\right)^{2}\right)+\frac{\lambda_{\mathrm{GB}}}{2}\left(\mathcal{R}^{2}-4 \mathcal{R}_{i j}^{2}+\mathcal{R}_{i j k l}^{2}-\frac{16}{d-2} \pi_{k}^{k} \mathcal{R}_{i j} \pi^{i j}\right. \\
& \quad+\frac{2 d}{(d-2)^{2}}\left(\pi_{i}^{i}\right)^{2} \mathcal{R}-2 \mathcal{R} \pi_{i j}^{2}+8 \mathcal{R}_{i j} \pi^{j k} \pi_{k}^{i}+4 \mathcal{R}_{i j k l} \pi^{i k} \pi^{j l}+2 \pi_{i}^{j} \pi_{j}^{k} \pi_{k}^{l} \pi_{l}^{i}-\left(\pi_{i j}^{2}\right)^{2} \\
&\left.\left.-\frac{16}{3(d-2)} \pi_{l}^{l} \pi_{i}^{j} \pi_{j}^{k} \pi_{k}^{i}+\frac{2 d}{(d-2)^{2}}\left(\pi_{k}^{k}\right)^{2} \pi_{i j}^{2}-\frac{3 d-4}{3(d-2)^{3}}\left(\pi_{i}^{i}\right)^{4}\right)\right]+O\left(\lambda_{\mathrm{GB}}^{2}\right)(5.26)
\end{aligned}
$$

\subsubsection{Recursive method}

Consider now a regularized space $\mathcal{M}_{r}$, whose boundary is $\Sigma_{r}$, with a fixed $r$ which in the end is meant to be taken to infinity. We add a generic boundary term $S_{b}$ to the action defined on this regularized space. In [24] it was shown that the variational problem is well defined if

$$
\left.S_{b}\right|_{r}=-\mathcal{S}_{r}
$$

where $\mathcal{S}_{r}$ is Hamilton's principal functional, given by the on-shell action with arbitrary boundary values for $\gamma_{i j}, \phi$, and $\chi$ on $\Sigma_{r}$.

It is well known that the canonical momenta can be obtained by taking functional derivatives of $\mathcal{S}_{r}$

$$
\pi^{i j}=\frac{\delta \mathcal{S}_{r}}{\delta \gamma_{i j}}, \quad \pi_{\phi}=\frac{\delta \mathcal{S}_{r}}{\delta \phi}, \quad \pi_{\chi}=\frac{\delta \mathcal{S}_{r}}{\delta \chi} .
$$

The Hamiltonian is constrained to vanish as a result of the equations of motion for $N$ and $N_{i}$

$$
H=0 \text {. }
$$

We can determine $\mathcal{S}_{r}$ by solving this constraint. The trick is to consider an expansion in 
eigenfunctions of the operator

$$
\delta_{\gamma}=\int_{\Sigma_{r}} d^{d-1} x 2 \gamma_{i j} \frac{\delta}{\delta \gamma_{i j}}
$$

One can verify that such an expansion is a derivative expansion

$$
\mathcal{S}_{r}=\mathcal{S}_{(0)}+\mathcal{S}_{(2)}+\mathcal{S}_{(4)}+\ldots
$$

with

$$
\delta_{\gamma} \mathcal{S}_{(2 n)}=(d-1-2 n) \mathcal{S}_{(2 n)} .
$$

Once we know the solution for $\mathcal{S}_{(0)}$, we can compute corrections to the action in a systematic way by solving algebraic equations. In fact, having to deal with algebraic equations instead of partial differential equations is the main advantage of the method of [24].

Now we write Hamilton's principal functional as

$$
\mathcal{S}_{r}=\int_{\Sigma_{r}} d^{d-1} x\left(\mathcal{L}_{(0)}+\mathcal{L}_{(2)}+\mathcal{L}_{(4)}+\ldots\right)
$$

From (5.28) we see that the canonical momenta also admit derivative expansions

$$
\begin{aligned}
& \pi^{i j}=\pi_{(0)}{ }^{i j}+\pi_{(2)}{ }^{i j}+\pi_{(4)}^{i j}+\ldots, \\
& \pi_{\phi}=\pi_{\phi(0)}+\pi_{\phi(2)}+\pi_{\phi(4)}+\ldots, \\
& \pi_{\chi}=\pi_{\chi(0)}+\pi_{\chi(2)}+\pi_{\chi(4)}+\ldots .
\end{aligned}
$$

Translating (5.32) in terms of the momenta and the Lagrangian density we obtain

$$
2 \pi_{(2 n)}=\frac{1}{\sqrt{-\gamma}}(d-1-2 n) \mathcal{L}_{(2 n)}
$$

where $\pi_{(2 n)}=\pi_{(2 n)}{ }_{i}$ is the trace taken with respect to $\gamma^{i j}$. This relation is crucial for the algorithm to work. Note that we can obtain all canonical momenta at some given order by just knowing the trace of the momentum conjugate to the induced metric.

We can now solve the Hamiltonian constraint $H=0$. Substituting the above expansions in the Hamiltonian and grouping terms with the same number of derivatives leads to an equation of the form

$$
H=H_{(0)}+H_{(2)}+H_{(4)}+\ldots=0
$$

which must be satisfied order by order, imposing separately $H_{(2 n)}=0$ for all $n$. 


\section{Solution at zeroth order}

We start by collecting terms with zero derivatives, which results in

$$
\begin{aligned}
H_{(0)}= & -(d-2)(d-1)-\pi_{(0)_{i j}}^{2}+\frac{\pi_{(0)}^{2}}{d-2}-\pi_{\phi(0)}^{2}-\frac{\pi_{\chi(0)}^{2}}{Z(\phi)} \\
& +\lambda_{\mathrm{GB}}\left(\frac{1}{2}\left(\pi_{(0)_{i j}}^{2}\right)^{2}-\frac{d \pi_{(0) i j}^{2} \pi_{(0)}^{2}}{(d-2)^{2}}+\frac{(3 d-4) \pi_{(0)}^{4}}{6(d-2)^{3}}-\pi_{(0)}{ }_{i}^{k} \pi_{(0)}^{i j}\left(\pi_{(0)}{ }_{j}^{l} \pi_{(0)} k l\right.\right. \\
& +O\left(\lambda_{\mathrm{GB}}^{2}\right) .
\end{aligned}
$$

Following [24], we try with the Ansatz for $\mathcal{L}_{(0)}$

$$
\mathcal{S}_{(0)}=2 \int_{\Sigma_{r}} d^{d-1} x \sqrt{-\gamma} \mathcal{W}(\phi, \chi)
$$

and compute the corresponding canonical momenta

$$
\pi_{(0)}^{i j}=\frac{\delta \mathcal{S}_{(0)}}{\delta \gamma_{i j}}=\gamma^{i j} \mathcal{W}, \quad \pi_{\phi(0)}=\frac{\delta \mathcal{S}_{(0)}}{\delta \phi}=2 \partial_{\phi} \mathcal{W}, \quad \pi_{\chi(0)}=\frac{\delta \mathcal{S}_{(0)}}{\delta \chi}=2 \partial_{\chi} \mathcal{W}
$$

Substituting into $H_{(0)}$ this gives

$$
\begin{aligned}
H_{(0)}=-(d-2)(d-1) & +\frac{(d-1) \mathcal{W}^{2}}{d-2}-\frac{4\left(\partial_{\chi} \mathcal{W}\right)^{2}}{Z(\phi)}-4\left(\partial_{\phi} \mathcal{W}\right)^{2} \\
& +\lambda_{\mathrm{GB}}\left(\frac{(d-4)(d-3)(d-1) \mathcal{W}^{4}}{6(d-2)^{3}}\right)+O\left(\lambda_{\mathrm{GB}}^{2}\right)
\end{aligned}
$$

We know from [24] that in the limit $\lambda_{\mathrm{GB}} \rightarrow 0$ the solution for $\mathcal{W}$ is the constant $d-2$. We can then write

$$
\mathcal{W}(\phi, \chi)=(d-2)+\lambda_{\mathrm{GB}} \mathcal{V}(\phi, \chi)+O\left(\lambda_{\mathrm{GB}}^{2}\right)
$$

Plugging (5.41) into (5.40) gives an equation for $\mathcal{V}(\phi, \chi)$, whose solution turns out to be also a constant

$$
\mathcal{V}=-\frac{1}{12}(d-4)(d-3)(d-2)
$$




\section{Solution at second order}

Terms with two derivatives can be collected into the following expression

$$
\begin{aligned}
& H_{(2)}=-\mathcal{R}-2 \pi_{(0)}^{i j} \pi_{(2)_{i j}}+\frac{2 \pi_{(0)} \pi_{(2)}}{d-2}-2 \pi_{\phi(0)} \pi_{\phi(2)}-\frac{2 \pi_{\chi_{(0)}} \pi_{\chi_{(2)}}}{Z(\phi)}+\frac{1}{2}\left(\partial_{i} \phi\right)^{2}+\frac{1}{2} Z(\phi)\left(\partial_{i} \chi\right)^{2} \\
& -\lambda_{\mathrm{GB}}\left[4 \mathcal{R}^{i j} \pi_{(0)}^{k} \pi_{(0) j k}+2 \mathcal{R}_{i k j l} \pi_{(0)}^{i j} \pi_{(0)}^{k l}+\frac{d \mathcal{R} \pi_{(0)}^{2}}{(d-2)^{2}}+\frac{2 d \pi_{(0)}^{i j} \pi_{(0)}^{2} \pi_{(2)}{ }_{i j}}{(d-2)^{2}}\right. \\
& -\frac{8 \pi_{(0) i}^{k} \pi_{(0)}^{i j} \pi_{(0)} \pi_{(2) j k}}{d-2}+4 \pi_{(0)}^{k} \pi_{(0)}^{i j} \pi_{(0)}^{l} \pi_{(2)} \pi_{k l}+\frac{8 \pi_{(0)}^{k} \pi_{(0)}^{i j} \pi_{(0) j k} \pi_{(2)}}{6-3 d}+\frac{8 \pi_{(0)}^{3} \pi_{(2)}}{3(d-2)^{3}} \\
& \left.-\frac{2 d \pi_{(0)}^{3} \pi_{(2)}}{(d-2)^{3}}-\pi_{(0)_{i j}}\left(\frac{8 \mathcal{R}^{i j} \pi_{(0)}}{d-2}+\pi_{(0)}^{i j}\left(\mathcal{R}+2 \pi_{(0)}^{k l} \pi_{(2)} k l-\frac{2 d \pi_{(0)} \pi_{(2)}}{(d-2)^{2}}\right)\right)\right]+O\left(\lambda_{\mathrm{GB}}^{2}\right) .
\end{aligned}
$$

Substitution of the zeroth order solution in $H_{(2)}$ leads to the following simple algebraic equation for $\pi_{(2)}$

$$
\left(-2+\left(1+(d-4)(d-3) \lambda_{\mathrm{GB}}\right) \mathcal{R}-\frac{1}{2}(d-4)(d-3) \lambda_{\mathrm{GB}}\right) \pi_{(2)}-\frac{1}{2}\left(\partial_{i} \phi\right)^{2}-\frac{1}{2} Z(\phi)\left(\partial_{i} \chi\right)^{2}=0 .
$$

Solving the above equation and using (5.35), we obtain $\mathcal{L}_{(2)}$

$$
\begin{aligned}
\mathcal{L}_{(2)}= & \frac{\sqrt{-\gamma}}{2(d-3)}\left(2 \mathcal{R}-\left(\partial_{i} \phi\right)^{2}-Z(\phi)\left(\partial_{i} \chi\right)^{2}+\lambda_{\mathrm{GB}} \frac{12-7 d+d^{2}}{4}\left(6 \mathcal{R}+\left(\partial_{i} \phi\right)^{2}+Z(\phi)\left(\partial_{i} \chi\right)^{2}\right)\right) \\
& +O\left(\lambda_{\mathrm{GB}}^{2}\right) .
\end{aligned}
$$

From this we can compute the momenta at second order

$$
\begin{aligned}
\pi_{(2)}^{i j}= & -\frac{1}{8(d-3)}\left[4\left(2 \mathcal{R}^{i j}-\partial^{i} \phi \partial^{j} \phi-Z(\phi) \partial^{i} \chi \partial^{j} \chi\right)-2 \gamma^{i j}\left(2 \mathcal{R}-\left(\partial_{k} \phi\right)^{2}-Z(\phi)\left(\partial_{k} \chi\right)^{2}\right)\right. \\
& \left.+\lambda_{\mathrm{GB}}\left(12-7 d+d^{2}\right)\left(6 \mathcal{R}^{i j}+\partial^{i} \phi \partial^{j} \phi+Z(\phi) \partial^{i} \chi \partial^{j} \chi-\frac{1}{2} \gamma^{i j}\left(6 \mathcal{R}+\left(\partial_{k} \phi\right)^{2}+Z(\phi)\left(\partial_{k} \chi\right)^{2}\right)\right)\right] \\
& +O\left(\lambda_{\mathrm{GB}}^{2}\right), \\
\pi_{\phi(2)}= & \frac{2-\frac{1}{2}\left(12-7 d+d^{2}\right) \lambda_{\mathrm{GB}}}{4(d-3)}\left(2 D_{i} D^{i} \phi-Z^{\prime}(\phi)\left(\partial_{i} \chi\right)^{2}\right)+O\left(\lambda_{\mathrm{GB}}^{2}\right), \\
\pi_{\chi(2)}= & \frac{2-\frac{1}{2}\left(12-7 d+d^{2}\right) \lambda_{\mathrm{GB}}}{2(d-3)}\left(Z(\phi) D_{i} D^{i} \chi+Z^{\prime}(\phi) \partial_{i} \chi \partial^{i} \phi\right)+O\left(\lambda_{\mathrm{GB}}^{2}\right) .
\end{aligned}
$$




\section{Solution at fourth order}

Finally, at fourth order we have

$$
\begin{aligned}
& H_{(4)}=-\pi_{(2)_{i j}} \pi_{(2)}^{i j}+\frac{\pi_{(2)}^{2}}{d-2}-\pi_{\phi(2)}^{2}-\frac{\pi_{\chi_{(2)}}^{2}}{Z(\phi)}-2 \pi_{(0)}^{i j} \pi_{(4)}{ }_{i j}+\frac{2 \pi_{(0)} \pi_{(4)}}{d-2}-2 \pi_{\phi(0)} \pi_{\phi(4)}-\frac{2 \pi_{\chi_{(0)}} \pi_{\chi_{(4)}}}{Z(\phi)} \\
& -\lambda_{\mathrm{GB}}\left[-2 \mathcal{R}_{i j} \mathcal{R}^{i j}+\frac{1}{2} \mathcal{R}^{2}+\frac{1}{2}\left(\mathcal{R}_{i j k l}\right)^{2}-2 \mathcal{R} \pi_{(0)}^{i j} \pi_{(2)_{i j}}-\frac{8 \mathcal{R}^{i j} \pi_{(0)} \pi_{(2)} i j}{d-2}+8 \mathcal{R}^{i j} \pi_{(0)_{i}}{ }^{k} \pi_{(2)_{j k}}\right. \\
& +\frac{d \pi_{(0)}^{2} \pi_{(2) i j} \pi_{(2)}^{i j}}{(d-2)^{2}}-\frac{8 \pi_{(0)}^{i j} \pi_{(0)} \pi_{(2)}{ }_{i} \pi_{(2) j k}}{d-2}+2 \pi_{(0)}^{i j} \pi_{(0)}^{k l} \pi_{(2) i k} \pi_{(2) j l}-2 \pi_{(0)}^{i j} \pi_{(0)}^{k l} \pi_{(2) i j} \pi_{(2)}{ }_{k l}
\end{aligned}
$$

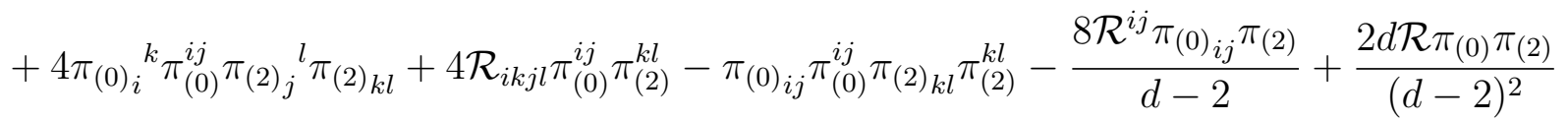

$$
\begin{aligned}
& +\frac{4 d \pi_{(0)}^{i j} \pi_{(0)} \pi_{(2) i j} \pi_{(2)}}{(d-2)^{2}}-\frac{8 \pi_{(0)}{ }^{k} \pi_{(0)}^{i j} \pi_{(2) j k} \pi_{(2)}}{d-2}+\frac{d \pi_{(0) i j} \pi_{(0)}^{i j} \pi_{(2)}^{2}}{(d-2)^{2}}+\frac{4 \pi_{(0)}^{2} \pi_{(2)}^{2}}{(d-2)^{3}}-\frac{3 d \pi_{(0)}^{2} \pi_{(2)}^{2}}{(d-2)^{3}} \\
& +\frac{\left.2 d \pi_{(0)}^{i j} \pi_{(0)}^{2} \pi_{(4)}\right)_{j j}}{(d-2)^{2}}-\frac{8 \pi_{(0)}^{k} \pi_{(0)}^{i j} \pi_{(0)} \pi_{(4)}{ }_{j k}}{d-2}+4 \pi_{(0)}{ }_{i}^{k} \pi_{(0)}^{i j} \pi_{(0)}^{l} \pi_{(4)}{ }_{k l}-2 \pi_{(0) i j} \pi_{(0)}^{i j} \pi_{(0)}^{k l} \pi_{(4) k l} \\
& \left.+\frac{8 \pi_{(0)}^{k} \pi_{(0)}^{i j} \pi_{(0)} \pi_{k} \pi_{(4)}}{6-3 d}+\frac{2 d \pi_{(0)}{ }_{i j} \pi_{(0)}^{i j} \pi_{(0)} \pi_{(4)}}{(d-2)^{2}}+\frac{8 \pi_{(0)}^{3} \pi_{(4)}}{3(d-2)^{3}}-\frac{2 d \pi_{(0)}^{3} \pi_{(4)}}{(d-2)^{3}}\right]+O\left(\lambda_{\mathrm{GB}}^{2}\right) .
\end{aligned}
$$

Now we repeat the previous steps. We substitute the zeroth and second order solutions in $H_{(4)}$, which leads to an algebraic equation for $\pi_{(4)}$ that can be readily solved. There is a subtlety due to the fact that the relation (5.35) is ill defined for $d=5$, which is our case of interest. In fact

$$
\mathcal{L}_{(4)}=\sqrt{-\gamma} \frac{2}{d-5} \pi_{(4)}
$$

However, the Hamilton-Jacobi method can still be applied (see the discussion in [24]) if we set the radial cut-off to be

$$
r=\frac{1}{d-5}
$$


and define $\tilde{\mathcal{L}}_{(4)}$ such that $\left.\mathcal{L}_{(4)}\right|_{r}=-\left.2 r \tilde{\mathcal{L}}_{(4)}\right|_{r}$, namely $\tilde{\mathcal{L}}_{(4)}=-\sqrt{-\gamma} \pi_{(4)}$. Proceeding in this way we finally arrive at

$$
\begin{aligned}
\frac{96}{r \sqrt{-\gamma}} \mathcal{L}_{(4)} & =-8 \mathcal{R}\left(\mathcal{R}-\left(\partial_{i} \phi\right)^{2}-Z(\phi) \partial_{i} \chi \partial^{i} \chi\right)+4\left(\partial_{i} \phi\right)^{4}+12 Z(\phi)\left(\partial_{i} \chi \partial^{i} \phi\right)^{2} \\
& -4 Z(\phi)\left(\partial_{i} \phi\right)^{2}\left(\partial_{j} \chi\right)^{2}+4 Z(\phi)^{2}\left(\partial_{i} \chi\right)^{4}+24 \mathcal{R}_{i j}\left(\mathcal{R}^{i j}-\partial^{i} \phi \partial^{j} \phi-Z(\phi) \partial^{i} \chi \partial^{j} \chi\right) \\
& +24\left(D_{i} D^{i} \phi-\frac{Z^{\prime}(\phi)}{2}\left(\partial_{i} \chi\right)^{2}\right)^{2}+24 Z(\phi)\left(D_{i} D^{i} \chi+\frac{Z^{\prime}(\phi)}{Z(\phi)}\left(\partial_{i} \chi \partial^{i} \phi\right)\right)^{2} \\
& +\lambda_{\mathrm{GB}}\left[76 \mathcal{R}^{2}+48 \mathcal{R}_{i j k l} \mathcal{R}^{i j k l}-12 \mathcal{R}\left(\left(\partial_{i} \phi\right)^{2}+Z(\phi)\left(\partial_{i} \chi\right)^{2}\right)+2\left(\partial_{i} \phi\right)^{4}+6 Z(\phi)\left(\partial_{i} \chi \partial^{i} \phi\right)^{2}\right. \\
& -2 Z(\phi)\left(\partial_{i} \phi\right)^{2}\left(\partial_{j} \chi\right)^{2}+2 Z(\phi)^{2}\left(\partial_{i} \chi\right)^{4}-12 \mathcal{R}_{i j}\left(23 \mathcal{R}^{i j}-3 \partial^{i} \phi \partial^{j} \phi-3 Z(\phi) \partial^{i} \chi \partial^{j} \chi\right) \\
& \left.-36\left(D_{i} D^{i} \phi-\frac{Z^{\prime}(\phi)}{2}\left(\partial_{i} \chi\right)^{2}\right)^{2}-36 Z(\phi)\left(D_{i} D^{i} \chi+\frac{Z^{\prime}(\phi)}{Z(\phi)} \partial_{i} \chi \partial^{i} \phi\right)^{2}\right]+O\left(\lambda_{\mathrm{GB}}^{2}\right) .
\end{aligned}
$$

Up to some overall factor, this expression coincides with the conformal anomaly, as we shall see in a moment.

\subsubsection{Fefferman-Graham expansions}

From the counterterms obtained using the Hamilton-Jacobi method we see that the canonical momenta take the form

$$
\begin{aligned}
& \pi^{i j}=\pi_{(0)}^{i j}+\pi_{(2)}^{i j}-2 r \tilde{\pi}_{(4)}^{i j}+\pi_{(4)}^{i j}+\ldots, \\
& \pi_{\phi}=\pi_{\phi(0)}+\pi_{\phi(2)}-2 r \tilde{\pi}_{\phi(4)}+\pi_{\phi(4)}+\ldots, \\
& \pi_{\chi}=\pi_{\chi(0)}+\pi_{\chi(2)}-2 r \tilde{\pi}_{\chi(4)}+\pi_{\chi(4)}+\ldots
\end{aligned}
$$

The fourth order terms $\pi_{(4)}^{i j}, \pi_{\phi_{(4)}}, \pi_{\chi_{(4)}}$ contain the information about the renormalized one-point functions. In order to determine these terms, we proceed with the asymptotic analysis. In Fefferman-Graham (FG) coordinates, the metric reads

$$
d s^{2}=\ell_{A d S}^{2}\left(\frac{d v^{2}}{v^{2}}+\gamma_{i j}(x, v) d x^{i} d x^{j}\right)
$$

The AdS radius $\ell_{A d S}$ is given by

$$
\ell_{A d S}=\frac{1}{\sqrt{F_{\mathrm{bdry}}}},
$$


and $v=e^{-r / \ell_{A d S}}$. Generically, the fields will have the following near-boundary expansions in these coordinates

$$
\begin{aligned}
\gamma_{i j} & =\frac{1}{v^{2}}\left(g_{(0) i j}+v^{2} g_{(2) i j}+v^{4}\left(g_{(4) i j}+2 h_{(4) i j} \log v\right)+O\left(v^{6}\right)\right) \\
\phi & =\phi_{(0)}+v^{2} \phi_{(2)}+v^{4}\left(\phi_{(4)}+2 \tilde{\phi}_{(4)} \log v\right)+O\left(v^{6}\right), \\
\chi & =\chi_{(0)}+v^{2} \chi_{(2)}+v^{4}\left(\chi_{(4)}+2 \tilde{\chi}_{(4)} \log v\right)+O\left(v^{6}\right) .
\end{aligned}
$$

The coefficients $g_{(0)}, \phi_{(0)}$ and $\chi_{(0)}$ remain undetermined from this analysis, but the other coefficients can be obtained as functions of $g_{(0)_{i j}}, \phi_{(0)}$ and $\chi_{(0)}$ by substituting the above expansions in (5.20) and comparing order by order in $v$. For example, comparing terms at order $O\left(v^{2}\right)$ we obtain

$$
\begin{aligned}
g_{(2) i j}= & -\frac{1-\lambda_{\mathrm{GB}}}{2} R_{i j}+\frac{1}{4}\left(1+\lambda_{\mathrm{GB}}\right)\left(\partial_{i} \phi_{(0)} \partial_{j} \phi_{(0)}+Z\left(\phi_{(0)}\right) \partial_{i} \chi_{(0)} \partial_{j} \chi_{(0)}\right) \\
& +\frac{1}{24} g_{(0)_{i j}}\left(2\left(1-\lambda_{\mathrm{GB}}\right) R-\left(1+\lambda_{\mathrm{GB}}\right)\left(\partial_{k} \phi_{(0)} \partial^{k} \phi_{(0)}+Z\left(\phi_{(0)}\right) \partial_{k} \chi_{(0)} \partial^{k} \chi_{(0)}\right)\right) \\
& +O\left(\lambda_{\mathrm{GB}}^{2}\right) \\
\phi_{(2)}= & \frac{1-\lambda_{\mathrm{GB}}}{8}\left(2 D_{(0) i} \partial^{i} \phi_{(0)}-Z^{\prime}\left(\phi_{(0)}\right) \partial_{i} \chi_{(0)} \partial^{i} \chi_{(0)}\right)+O\left(\lambda_{\mathrm{GB}}^{2}\right) \\
\chi_{(2)}= & \frac{1-\lambda_{\mathrm{GB}}}{4 Z\left(\phi_{(0)}\right)}\left(Z\left(\phi_{(0)}\right) D_{(0) i} \partial^{i} \chi_{(0)}+Z^{\prime}\left(\phi_{(0)}\right) \partial_{i} \chi_{(0)} \partial^{i} \phi_{(0)}\right)+O\left(\lambda_{\mathrm{GB}}^{2}\right) .
\end{aligned}
$$

Here and in the following the curvatures $R$ and $R_{i j}$ are the ones for $g_{(0) i j}$. Comparing the logarithmic term, we obtain instead

$$
\begin{aligned}
\tilde{\pi}_{(4) i j} & =2\left(1-\lambda_{\mathrm{GB}}\right)\left(h_{(4)_{i j}}-h_{(4)}{ }_{k} g_{(0)_{i j}}\right)+O\left(\lambda_{\mathrm{GB}}^{2}\right), \\
\tilde{\phi}_{(4)} & =\frac{1}{4}\left(1-\lambda_{\mathrm{GB}}\right) \tilde{\pi}_{\phi(4)}+O\left(\lambda_{\mathrm{GB}}^{2}\right), \quad \tilde{\chi}_{(4)}=\frac{1-\lambda_{\mathrm{GB}}}{4 Z\left(\phi_{(0)}\right)} \tilde{\pi}_{\chi(4)}+O\left(\lambda_{\mathrm{GB}}^{2}\right) .
\end{aligned}
$$

\subsubsection{The 1-point functions}

The order $O\left(v^{4}\right)$ leads to the following renormalized one-point functions, which represent the main result of our analysis in this section. For the stress tensor we get

$$
\begin{aligned}
\left\langle T_{i j}\right\rangle= & 2 \pi_{(4)_{i j}} \\
= & -2 g_{(2)_{i j}} g_{(2)}{ }_{k}{ }_{k}+4 g_{(4)}+2 h_{(4)_{i j}}+2 g_{(2)_{k l}} g_{(2)}{ }^{k l} g_{(0)_{i j}}-4 g_{(4)}{ }_{k}{ }_{k} g_{(0)_{i j}}-2 h_{(4)}{ }_{k} g_{(0)_{i j}} \\
& +\frac{1}{2} g_{(2)}{ }^{k l} g_{(0) i j} R_{k l}-\frac{1}{2} g_{(2) i j} R-\frac{1}{2} D_{(0)_{i}} \phi_{(2)} D_{(0) j} \phi_{(0)}-\frac{1}{2} D_{(0) i} \phi_{(0)} D_{(0)} \phi_{(2)}
\end{aligned}
$$




$$
\begin{aligned}
& -\frac{Z\left(\phi_{(0)}\right)}{2} D_{(0)} \chi_{i} \chi_{(2)} D_{(0)} \chi_{(0)}+\frac{Z\left(\phi_{(0)}\right)}{2} g_{(0)}{ }_{i j} D_{(0)} \chi_{k} \chi_{(2)} D_{(0)}{ }^{k} \chi_{(0)} \\
& -\frac{Z\left(\phi_{(0)}\right)}{2} D_{(0)} \chi_{(0)} D_{(0) j} \chi_{(2)}-\frac{1}{2} D_{(0)}{ }_{j} D_{(0)} g_{(2)}{ }_{k}+\frac{1}{2} D_{(0)} D_{(0) i} g_{(2)}{ }^{k} \\
& +\frac{1}{2} D_{(0)} D_{(0)} g_{(2)}{ }_{i}{ }^{k}-\frac{1}{2} D_{(0) k} D_{(0)}^{k} g_{(2)}{ }_{i j}+\frac{1}{4} g_{(2)}{ }_{i j} D_{(0)} \phi_{(0)} D_{(0)}{ }^{k} \phi_{(0)} \\
& +\frac{1}{2} g_{(0)}{ }_{i j} D_{(0)} \phi_{(2)} D_{(0)}{ }^{k} \phi_{(0)}+\frac{Z\left(\phi_{(0)}\right)}{4} g_{(2)_{i j}} D_{(0)} \chi_{(0)} D_{(0)}{ }^{k} \chi_{(0)} \\
& -\frac{1}{2} g_{(0)}{ }_{i j} D_{(0)} D_{(0)}{ }_{k} g_{(2)}{ }^{k l}+\frac{1}{2} g_{(0)_{i j}} D_{(0) \ell} D_{(0)}^{\ell} g_{(2)}{ }_{k}{ }_{k}-\frac{1}{4} g_{(2)}{ }_{k l} g_{(0)}{ }_{i j} D_{(0)}{ }^{k} \phi_{(0)} D_{(0)}{ }^{l} \phi_{(0)} \\
& -\frac{Z\left(\phi_{(0)}\right)}{4} g_{(2)}{ }_{k l} g_{(0)}{ }_{i j} D_{(0)}{ }^{k} \chi_{(0)} D_{(0)}{ }^{l} \chi_{(0)}-\frac{Z^{\prime}\left(\phi_{(0)}\right)}{2} \phi_{(2)} D_{(0)} \chi_{(0)} D_{(0)} \chi_{(0)} \\
& +\frac{Z^{\prime}\left(\phi_{(0)}\right)}{4} g_{(0)}{ }_{i j} \phi_{(2)} D_{(0)} \chi_{(0)} D_{(0)}{ }^{k} \chi_{(0)}+\lambda_{\mathrm{GB}} T_{\mathrm{GB} i j}+O\left(\lambda_{\mathrm{GB}}^{2}\right),
\end{aligned}
$$

where

$$
\begin{aligned}
& T_{\mathrm{GB} i j}=-4 g_{(2)_{i}}{ }^{k} g_{(2)_{j k}}+7 g_{(2)_{i j}} g_{(2)}{ }_{k}{ }_{k}-6 g_{(4)_{i j}}-3 h_{(4)_{i j}}-g_{(2)}{ }_{k l} g_{(2)}{ }^{k l} g_{(0)_{i j}} \\
& -2\left(g_{(2)}{ }_{k}{ }^{2}\right)^{2} g_{(0)_{i j}}+6 g_{(4)}{ }_{k}{ }_{k} g_{(0)_{i j}}+3 h_{(4)}{ }_{k} g_{(0)_{i j}}+\frac{13}{4} g_{(2)_{i j}} R-2 g_{(2)}{ }_{k} g_{(0)_{i j}} R \\
& +\frac{29}{2} g_{(2)}{ }^{k l} R_{i k j l}+4 g_{(2)}{ }_{k}^{k} R_{i j}-\frac{53}{4} g_{(2)}{ }_{j}{ }^{k} R_{i k}-\frac{53}{4} g_{(2)}{ }^{k} R_{j k}+\frac{11}{4} g_{(2)}{ }^{k l} g_{(0)}{ }_{i j} R_{k l} \\
& +\frac{1}{4} D_{(0)} \phi_{(2)} D_{(0)} \phi_{(0)}+\frac{1}{4} D_{(0)} \phi_{(0)} D_{(0)} \phi_{(2)}+\frac{Z\left(\phi_{(0)}\right)}{4} D_{(0)} \chi_{(2)} D_{(0)} \chi_{(0)} \\
& +\frac{Z\left(\phi_{(0)}\right)}{4} D_{(0)} \chi_{(0)} D_{(0)} \chi_{(2)}+\frac{37}{4} D_{(0)}{ }_{j} D_{(0)} g_{(2)}{ }_{k}{ }_{k}-\frac{37}{4} D_{(0)} D_{(0)} g_{(2)}{ }_{j} \\
& -\frac{37}{4} D_{(0)} D_{(0)} g_{(2) i}^{k}+\frac{37}{4} D_{(0) k} D_{(0)}^{k} g_{(2)_{i j}}-\frac{1}{8} g_{(2)_{i j}} D_{(0)} \phi_{(0)} D_{(0)}{ }^{k} \phi_{(0)} \\
& -\frac{1}{4} g_{(0)_{i j}} D_{(0)} \phi_{(2)} D_{(0)}{ }^{k} \phi_{(0)}-\frac{Z\left(\phi_{(0)}\right)}{8} g_{(2)}{ }_{i j} D_{(0)} \chi_{(0)} D_{(0)}{ }^{k} \chi_{(0)} \\
& -\frac{Z\left(\phi_{(0)}\right)}{4} g_{(0)}{ }_{i j} D_{(0)} \chi_{(2)} D_{(0)}{ }^{k} \chi_{(0)}-\frac{Z^{\prime}\left(\phi_{(0)}\right)}{8} g_{(0)}{ }_{i j} \phi_{(2)} D_{(0)}{ }_{k} \chi_{(0)} D_{(0)}{ }^{k} \chi_{(0)} \\
& +\frac{5}{4} g_{(0)_{i j}} D_{(0)} D_{(0)} g_{(2)}{ }^{k l}-\frac{5}{4} g_{(0)}{ }_{i j} D_{(0) \ell} D_{(0)}^{\ell} g_{(2)}{ }_{k}{ }_{k}+\frac{1}{8} g_{(2)}{ }_{k l} g_{(0)}{ }_{i j} D_{(0)}{ }^{k} \phi_{(0)} D_{(0)}{ }^{l} \phi_{(0)} \\
& +\frac{Z\left(\phi_{(0)}\right)}{8} g_{(2) k l} g_{(0)} D_{i j} D_{(0)}^{k} \chi_{(0)} D_{(0)}{ }^{l} \chi_{(0)}+\frac{Z^{\prime}\left(\phi_{(0)}\right)}{4} \phi_{(2)} D_{(0)} \chi_{(0)} D_{(0)} \chi_{(0)} \text {. }
\end{aligned}
$$

For the dilaton and axion we get instead

$$
\begin{aligned}
& \left\langle\mathcal{O}_{\phi}\right\rangle=-\pi_{\phi(4)} \\
& =-\left(2+\lambda_{\mathrm{GB}}\right)\left(2 \phi_{(4)}+\tilde{\phi}_{(4)}\right) \\
& +\frac{1}{4}\left(2-\lambda_{\mathrm{GB}}\right)\left[D_{(0) i} D_{(0)}^{i} \phi_{(2)}+\frac{1}{2} D_{(0)} g_{(2)}{ }_{j}{ }_{j} D_{(0)}{ }^{i} \phi_{(0)}-D_{(0)}{ }^{i} \phi_{(0)} D_{(0)} g_{(2)}{ }_{i}\right. \\
& -g_{(2)_{i j}} D_{(0)}^{j} D_{(0)}{ }^{i} \phi_{(0)}+\frac{Z^{\prime}\left(\phi_{(0)}\right)}{2} g_{(2)_{i j}} D_{(0)}{ }^{i} \chi_{(0)} D_{(0)}{ }^{j} \chi_{(0)} \\
& \left.-Z^{\prime}\left(\phi_{(0)}\right) D_{(0)} \chi_{(2)} D_{(0)}{ }^{i} \chi_{(0)}-\frac{Z^{\prime \prime}\left(\phi_{(0)}\right)}{2} \phi_{(2)} D_{(0)} \chi_{(0)} D_{(0)}{ }^{i} \chi_{(0)}\right] \\
& +O\left(\lambda_{\mathrm{GB}}^{2}\right),
\end{aligned}
$$


and

$$
\begin{aligned}
\left\langle\mathcal{O}_{\chi}\right\rangle=- & -\pi_{\chi(4)} \\
- & -\left(2+\lambda_{\mathrm{GB}}\right)\left(2 Z\left(\phi_{(0)}\right) \chi_{(4)}+Z\left(\phi_{(0)}\right) \tilde{\chi}_{(4)}+Z^{\prime}\left(\phi_{(0)}\right) \phi_{(2)} \chi_{(2)}\right) \\
+ & +\frac{1}{4}\left(2-\lambda_{\mathrm{GB}}\right)\left[Z\left(\phi_{(0)}\right) D_{(0) i} D_{(0)}^{i} \chi_{(2)}+\frac{Z\left(\phi_{(0)}\right.}{2} D_{(0) i} g_{(2)}{ }_{j}{ }_{j} D_{(0)}{ }^{i} \chi_{(0)}\right. \\
& -Z\left(\phi_{(0)}\right) D_{(0)}{ }^{i} \chi_{(0)} D_{(0)} g_{(2) i}{ }^{j}-Z\left(\phi_{(0)}\right) g_{(2) i j} D_{(0)}{ }^{j} D_{(0)}{ }^{i} \chi_{(0)} \\
& +Z^{\prime}\left(\phi_{(0)}\right) \phi_{(2)} D_{(0) i} D_{(0)}^{i} \chi_{(0)}+Z^{\prime}\left(\phi_{(0)}\right) D_{(0)} \chi_{(2)} D_{(0)}{ }^{i} \phi_{(0)} \\
& +Z^{\prime}\left(\phi_{(0)}\right) D_{(0) i} \chi_{(0)} D_{(0)}{ }^{i} \phi_{(2)}-Z^{\prime}\left(\phi_{(0)}\right) g_{(2) i j} D_{(0)}{ }^{i} \phi_{(0)} D_{(0)}{ }^{j} \chi_{(0)} \\
& \left.+Z^{\prime \prime}\left(\phi_{(0)}\right) \phi_{(2)} D_{(0)} \chi_{(0)} D_{(0)}{ }^{i} \phi_{(0)}\right]
\end{aligned}
$$

We stress that these formulas are generic for any axion-dilaton system with GB term (to first order in $\lambda_{\mathrm{GB}}$ ) of the structure given in (5.19).

The zeroth order terms in $\lambda_{\mathrm{GB}}$ in these expressions reproduce the results of [24], while the first order terms in $\lambda_{\mathrm{GB}}$ extend the results of [25] to a system with an axion-dilaton field. As mentioned already, another difference with the analysis of [25] is that we employ a recursive method which is more effective in cases where multiple fields, besides the metric, are turned on.

\subsubsection{Central charges}

The trace of the stress energy tensor is related to the central charges $a$ and $c$ by the following expression 6

$$
\left\langle T_{i}^{i}\right\rangle=\frac{1}{16 \pi^{2}}(c W-a E)+\ldots,
$$

where $E$ is the four-dimensional Euler density

$$
E=\mathcal{R}^{2}-4 \mathcal{R}_{i j} \mathcal{R}^{i j}+\mathcal{R}_{i j k l} \mathcal{R}^{i j k l},
$$

$W$ is the square of the Weyl tensor

$$
W=C^{i j k l} C_{i j k l}=\frac{\mathcal{R}^{2}}{3}-2 \mathcal{R}_{i j} \mathcal{R}^{i j}+\mathcal{R}_{i j k l} \mathcal{R}^{i j k l}
$$

and where the ellipsis indicates the contribution by other fields (the axion-dilaton in our specific setting). The trace of the stress energy tensor [24] is given by the $\mathcal{L}_{(4)}$ written

\footnotetext{
${ }^{6}$ Notice that in this section $a$ denotes one of the central charges and not the anisotropy parameter.
} 
above in $(5.50)$

$$
\left\langle T_{i}^{i}\right\rangle=\frac{1}{r \sqrt{-\gamma}} \mathcal{L}_{(4)}
$$

To isolate the metric contribution we set $\phi=\chi=0$ in that expression and arrive at

$$
\left\langle T_{i}^{i}\right\rangle=-\frac{1}{12} \mathcal{R}^{2}+\frac{1}{4} \mathcal{R}_{i j} \mathcal{R}^{i j}+\left(\frac{19}{24} \mathcal{R}^{2}+\frac{1}{2} \mathcal{R}_{i j k l} \mathcal{R}^{i j k l}-\frac{23}{8} \mathcal{R}_{i j} \mathcal{R}^{i j}\right) \lambda_{\mathrm{GB}}+O\left(\lambda_{\mathrm{GB}}^{2}\right)
$$

Comparing (5.61) and (5.65), we find that

$$
a=\pi^{2}\left(2-15 \lambda_{\mathrm{GB}}\right)+O\left(\lambda_{\mathrm{GB}}^{2}\right), \quad c=\pi^{2}\left(2-7 \lambda_{\mathrm{GB}}\right)+O\left(\lambda_{\mathrm{GB}}^{2}\right),
$$

thus confirming that indeed $a \neq c$ for theories with GB corrections. These results are in perfect agreement with previous literature, see e.g. [112].

\subsection{Boundary stress tensor}

Here we specialize the formulas above to our solution (5.8). As a first step, we need to rewrite the fields in FG coordinates, to be able to extract the asymptotic behaviors close to the boundary.

We define the FG radial coordinate $v$ such that ${ }^{7}$

$$
\frac{d u^{2}}{u^{2} F(u)}=\frac{d v^{2}}{v^{2} F_{\mathrm{bdry}}}+O\left(v^{3}\right), \quad F_{\mathrm{bdry}}=\frac{1-\sqrt{1-4 \lambda_{\mathrm{GB}}}}{2 \lambda_{\mathrm{GB}}}
$$

The relation between the two radial coordinates $u$ and $v$ turns out to be given explicitly by

$$
\begin{aligned}
u=v+\frac{1}{48} a^{2}\left(\lambda_{\mathrm{GB}}+1\right) v^{3} & \\
& \quad-\frac{a^{2} u_{\mathrm{H}}^{2}\left(2 \lambda_{\mathrm{GB}}(\log 32-1)+1+\log 4\right)+12\left(\lambda_{\mathrm{GB}}+1\right)}{96 u_{\mathrm{H}}^{4}} v^{5}+O\left(a^{4}, \lambda_{\mathrm{GB}}^{2}, v^{7}\right) .
\end{aligned}
$$

In terms of $v$ the fields have the following asymptotic expansions

$$
\begin{aligned}
& \phi(v)=-\frac{a^{2}}{4}\left(1-\lambda_{\mathrm{GB}}\right) v^{2}+\frac{a^{2}}{8 u_{\mathrm{H}}^{2}}\left(1-\lambda_{\mathrm{GB}}\right) v^{4}+O\left(v^{6}\right), \\
& F(v)=1+\lambda_{\mathrm{GB}}+\frac{a^{2}}{12}\left(1+2 \lambda_{\mathrm{GB}}\right) v^{2}
\end{aligned}
$$

\footnotetext{
${ }^{7}$ Asymptotically, our metric approaches $A d S_{5}$ with curvature radius given by $\ell_{A d S}=1 / \sqrt{F_{\mathrm{bdry}}}$, which explains the factor of $F_{\mathrm{bdry}}$ in the formula, see (5.52).
} 


$$
\begin{gathered}
-\left(\frac{1+2 \lambda_{\mathrm{GB}}}{u_{\mathrm{H}}^{4}}+\frac{a^{2}}{12 u_{\mathrm{H}}^{2}}\left(1+2 \log 2-(1-12 \log 2) \lambda_{\mathrm{GB}}\right)\right) v^{4}+O\left(v^{6}\right), \\
B(v)=1-\lambda_{\mathrm{GB}}-\frac{a^{2}}{12} v^{2}+\frac{a^{2}}{8 u_{\mathrm{H}}^{2}} v^{4}+O\left(v^{6}\right) \\
H(v)=1+\frac{a^{2}}{4}\left(1+\lambda_{\mathrm{GB}}\right) v^{2}-\frac{a^{2}}{8 u_{\mathrm{H}}^{2}}\left(1+\lambda_{\mathrm{GB}}\right) v^{4}+O\left(v^{6}\right)
\end{gathered}
$$

From these expressions it is easy to find the expansions for the metric

$$
\begin{aligned}
g_{t t}=-1 & +\frac{a^{2}}{24}\left(1+\lambda_{\mathrm{GB}}\right) v^{2} \\
& +\frac{1}{16 u_{\mathrm{H}}^{4}}\left(12\left(1+\lambda_{\mathrm{GB}}\right)-a^{2} u_{\mathrm{H}}^{2}\left(1-2 \log 2+2 \lambda_{\mathrm{GB}}(2-5 \log 2)\right)\right) v^{4}+O\left(v^{6}\right), \\
g_{x x}=g_{y y}=1-\frac{a^{2}}{24}\left(1+\lambda_{\mathrm{GB}}\right) v^{2} & +\frac{1}{48 u_{\mathrm{H}}^{4}}\left(12\left(1+\lambda_{\mathrm{GB}}\right)+a^{2} u_{\mathrm{H}}^{2}\left(1+2 \log 2-2 \lambda_{\mathrm{GB}}(1-5 \log 2)\right)\right) v^{4}+O\left(v^{6}\right), \\
g_{z z}=1+ & \frac{5 a^{2}}{24}\left(1+\lambda_{\mathrm{GB}}\right) v^{2} \\
& +\frac{1}{48 u_{\mathrm{H}}^{4}}\left(12\left(1+\lambda_{\mathrm{GB}}\right)-a^{2} u_{\mathrm{H}}^{2}\left(5-2 \log 2+2 \lambda_{\mathrm{GB}}(4-5 \log 2)\right)\right) v^{4}+O\left(v^{6}\right),
\end{aligned}
$$

from which it is immediate to extract $g_{(2) i j}$ and $g_{(4) i j}$.

In our solution the terms up to $O\left(a^{2}\right)$ are very simple:

$$
\left\langle T_{i j}\right\rangle=4 g_{(4)_{i j}}-6 \lambda_{\mathrm{GB}} g_{(4)}, \quad \pi_{\phi(4)}=0, \quad \pi_{\chi_{(4)}}=0
$$

Explicitly, the components of the stress tensor read

$$
\begin{aligned}
\left\langle T_{t t}\right\rangle & =\frac{3}{u_{\mathrm{H}}^{4}}-\frac{1-2 \log 2}{4 u_{\mathrm{H}}^{2}} a^{2}-\frac{12+a^{2} u_{\mathrm{H}}^{2}(5-14 \log 2)}{8 u_{\mathrm{H}}^{4}} \lambda_{\mathrm{GB}}+O\left(a^{4}, \lambda_{\mathrm{GB}}^{2}\right), \\
\left\langle T_{x x}\right\rangle & =\left\langle T_{y y}\right\rangle=\frac{1}{u_{\mathrm{H}}^{4}}+\frac{1+2 \log 2}{12 u_{\mathrm{H}}^{2}} a^{2}-\frac{12+a^{2} u_{\mathrm{H}}^{2}(7-14 \log 2)}{24 u_{\mathrm{H}}^{4}} \lambda_{\mathrm{GB}}+O\left(a^{4}, \lambda_{\mathrm{GB}}^{2}\right), \\
\left\langle T_{z z}\right\rangle & =\frac{1}{u_{\mathrm{H}}^{4}}-\frac{5-2 \log 2}{12 u_{\mathrm{H}}^{2}} a^{2}-\frac{12+a^{2} u_{\mathrm{H}}^{2}(1-14 \log 2)}{24 u_{\mathrm{H}}^{4}} \lambda_{\mathrm{GB}}+O\left(a^{4}, \lambda_{\mathrm{GB}}^{2}\right) .
\end{aligned}
$$

Using (5.13) we see that

$$
u_{\mathrm{H}}=\frac{1}{\pi T}-\frac{1-\log 2}{24 \pi^{3} T^{3}} a^{2}-\frac{1}{2 \pi T}\left(1-\frac{5 \log 2}{24 \pi^{2} T^{2}} a^{2}\right) \lambda_{\mathrm{GB}}+O\left(a^{4}, \lambda_{\mathrm{GB}}^{2}\right),
$$

so that we can rewrite the expressions above in terms of the temperature, which is a 
physical observable, unlike the horizon location $u_{\mathrm{H}}$. We arrive at our final results:

$$
\begin{aligned}
\left\langle T_{t t}\right\rangle & =3 \pi^{4} T^{4}\left[1+\frac{1}{12 \pi^{2}}\left(\frac{a}{T}\right)^{2}+\left(\frac{3}{2}+\frac{1}{24 \pi^{2}}\left(\frac{a}{T}\right)^{2}\right) \lambda_{\mathrm{GB}}\right]+O\left(a^{4}, \lambda_{\mathrm{GB}}^{2}\right), \\
\left\langle T_{x x}\right\rangle & =\left\langle T_{y y}\right\rangle=\pi^{4} T^{4}\left[1+\frac{1}{4 \pi^{2}}\left(\frac{a}{T}\right)^{2}+\left(\frac{3}{2}+\frac{1}{8 \pi^{2}}\left(\frac{a}{T}\right)^{2}\right) \lambda_{\mathrm{GB}}\right]+O\left(a^{4}, \lambda_{\mathrm{GB}}^{2}\right), \\
\left\langle T_{z z}\right\rangle & =\pi^{4} T^{4}\left[1-\frac{1}{4 \pi^{2}}\left(\frac{a}{T}\right)^{2}+\left(\frac{3}{2}-\frac{1}{8 \pi^{2}}\left(\frac{a}{T}\right)^{2}\right) \lambda_{\mathrm{GB}}\right]+O\left(a^{4}, \lambda_{\mathrm{GB}}^{2}\right) .
\end{aligned}
$$

These quantities correspond to the energy density and pressures of the dual gauge theory

$$
E=\frac{N_{\mathrm{c}}^{2}}{8 \pi^{2}}\left\langle T_{t t}\right\rangle, \quad P_{\perp}=\frac{N_{\mathrm{c}}^{2}}{8 \pi^{2}}\left\langle T_{x x}\right\rangle, \quad P_{\|}=\frac{N_{\mathrm{c}}^{2}}{8 \pi^{2}}\left\langle T_{z z}\right\rangle,
$$

with $N_{\mathrm{c}}$ being the number of colors of the gauge theory and $P_{\perp}$ and $P_{\|}$the pressures along the transverse plane and the longitudinal direction, respectively. The comparison with the energy density $E_{0}(T)=3 \pi^{2} N_{\mathrm{c}}^{2} T^{4} / 8$ and the pressure $P_{0}(T)=\pi^{2} N_{\mathrm{c}}^{2} T^{4} / 8$ of an isotropic plasma at the same temperature and $\lambda_{\mathrm{GB}}=0$ is obvious from the expressions above. We see in particular that the anisotropy has the effect of increasing the energy density and perpendicular pressure compared to the isotropic case, while decreasing the longitudinal pressure. This is consistent with the findings of $[22,23]$ in the small anisotropy limit (whose results we reproduce for $\lambda_{\mathrm{GB}}=0$, see eq. (168) of [23]).

These results show that the system is really anisotropic in the $z$-direction, as $P_{\perp} \neq P_{\|}$. Notice that at this order in $a$, the trace of the stress tensor is vanishing

$$
\left\langle T_{i}^{i}\right\rangle=O\left(a^{4}, \lambda_{\mathrm{GB}}^{2}\right)
$$

This is in agreement with what found in [22,23], where the conformal anomaly was also vanishing at order $O\left(a^{2}\right)$ and appearing only at order $O\left(a^{4}\right)$ and beyond. We can also check some basic thermodynamic relations. In particular, the free energy $\mathcal{F}=E-T s$, in the limit of $a=0$, matches perfectly the value found in [97] from evaluating the Euclidean action on-shell. We can also check that $\mathcal{F}=-P_{\perp}$, as it should be [113].

Finally, let us comment about the conservation of the (expectation value of the) stress tensor. Remember that to simplify our expressions we have gauge fixed the lapse and shift functions (see footnote 5). As a consequence we can no longer derive the diffeomorphism Ward identity that relates the divergence of $\left\langle T_{i j}\right\rangle$ to the expectation values of the other fields. Typically, in such an identity we expect a term of the form $\left\langle\mathcal{O}_{\chi}\right\rangle \partial_{j} \chi_{(0)}$, see e.g. eq. (B.20) of [24]. In the particular state we have considered, even though $\partial_{j} \chi_{(0)} \neq 0$, we do have that $\left\langle\mathcal{O}_{\chi}\right\rangle=0$, see (5.71). This contribution would then vanish, assuring that $D_{(0)}^{i}\left\langle T_{i j}\right\rangle=0$ and guaranteeing the translational invariance of the geometry. For a more detailed study of the thermodynamics of this system, which is beyond the scope of the 
present work, one would need to derive this Ward identity.

\subsection{Discussion}

In this work we have explored the effects of higher curvature corrections (given by the inclusion of a GB term) in a system of AdS-gravity in five dimensions coupled to an axion-dilaton field. As we have explained above, these corrections correspond, on the gauge theory side, to considering cases that are more generic than the ones usually considered, e.g. conformal field theories with independent central charges, $a \neq c$. It is still unclear whether our setup might be obtained in the low energy limit of some string theory, and our philosophy has been 'bottom-up'.

One of our main concerns has been to carry out holographic renormalization and compute the 1-point function of the boundary stress tensor associated to our gravitational theory. We have done this to first order in the GB coupling, which is however not a terribly restrictive constraint, since requirements of unitarity, causality and positivity of energy fluxes require [13]

$$
-7 / 36 \leq \lambda_{\mathrm{GB}} \leq 9 / 100
$$

We have also considered a particular black brane Ansatz, in which the axion field is linearly dependent on one of the horizon coordinates, while being independent of the radial coordinate. This has resulted in finding an anisotropic black brane solution to the equations of motion, which is the GB-corrected equivalent of the geometry discovered in $[22,23]$. One point that remains to be settled in our analysis is whether our solution might be interpreted as an interpolating solution between a Lifshitz-like scaling solution in the IR and an asymptotically AdS space, as was the case for the $\lambda_{\mathrm{GB}}=0$ limit of $[22,23]$.

One of the most interesting applications of the present work would be a detailed study of the thermodynamics of this black brane and of its corresponding plasma. This analysis was carried out, in the canonical ensemble, for the case of vanishing $\lambda_{\mathrm{GB}}$ in $[22,23]$ and a rich phase diagram was discovered, with, in particular, the presence of instabilities that might turn out to be useful in understanding the fast thermalization time of the QGP. To this regard it is relevant to observe that part of the richness of the solution in [22,23] was due to a conformal anomaly, appearing in the renormalization process at order $O\left(a^{4}\right)$ and beyond. In the present solution we also have an anomaly, which we expect to appear at the fourth order in the anisotropy parameter, but we are not able to capture with our analytic solution, which only goes up to second order. Extending our analytic solution to order $O\left(a^{4}\right)$ seems unviable and presumably numerical methods would have to be employed to explore larger values of the anisotropy. Given the large number of parameters in the game, this might be cumbersome, but it surely is something worth pursuing. 


\section{Chapter 6}

\section{Probing strongly coupled anisotropic plasmas from higher curvature gravity}

One application of the solution we have found in the previous chapter is to model higher curvature effects on the dual gauge theory plasma. Generically, heavy ion collisions experiments are non-central, resulting in a spatial anisotropy of the QGP formed after the collision. This represents one of the main motivations for our Ansatz. In the following we will identify $z$ as the anisotropic direction (or 'beam' direction), while $x$ and $y$ parametrize the plane transverse to the beam.

Our aim is to study qualitatively how several observables relevant to the study of the QGP are affected by the parameters $\left(a, \lambda_{\mathrm{GB}}\right)$. Anticipating the results of our analysis, we found that the effect of the Gauss-Bonnet term in the observables of the gauge theory is consistent with our physical intuition regarding the QGP as a fluid, interpreting the results in terms of the mean free path which is associated to the shear viscosity.

We compute several observables relevant to the study of the QGP, namely, the shear viscosity over entropy density, the drag force experienced by a heavy quark moving through the plasma, the jet quenching parameter, the static potential between a quark-antiquark pair (quarkonium) and the photon production rate. Most part of the analysis of our results involves a comparison with the isotropic $\mathcal{N}=4$ SYM result, obtained by taking $a \rightarrow 0$ and $\lambda_{\mathrm{GB}} \rightarrow 0$. We limit ourselves to the comparison at the same temperature, for simplicity, but a comparison at the same entropy density is still possible, and it was done for the observables computed in the model of $[22,23]$. We summarize and discuss our results in Section 6.7. For completeness, Appendix C presents an alternative derivation of the shear viscosity using the Kubo formula, and Appendices D, E, and F contain the derivation of the main formulas used in this work. 


\subsection{Gravity setup}

For easier reference we repeat the relevant aspects of the gravity solution we have found in the previous in Chapter 5. The Ansatz for the metric and scalar fields takes the form

$$
\begin{gathered}
d s^{2}=G_{m n} d x^{m} d x^{n}=\frac{1}{u^{2}}\left(-F(u) B(u) d t^{2}+d x^{2}+d y^{2}+H(u) d z^{2}+\frac{d u^{2}}{F(u)}\right), \\
\chi=a z, \quad \phi=\phi(u) .
\end{gathered}
$$

The axion field introduces a spatial anisotropy in the $z$-direction controlled by the anisotropy parameter $a$. An analytical solution to the equations of motion can be obtained in the limit of small anisotropy. Their expressions take the form

$$
\begin{aligned}
\phi(u) & =a^{2} \phi_{2}(u)+O\left(a^{4}\right), \\
F(u) & =F_{0}(u)+a^{2} F_{2}(u)+O\left(a^{4}\right), \\
B(u) & =B_{0}\left(1+a^{2} B_{2}(u)+O\left(a^{4}\right)\right), \\
H(u) & =1+a^{2} H_{2}(u)+O\left(a^{4}\right) .
\end{aligned}
$$

The explicit expressions for the functions $\phi_{2}, F_{2}, B_{2}$ and $H_{2}$ can be found in Appendix B. The leading terms $F_{0}(u)$ and $B_{0}$ are known from pure Gauss-Bonnet gravity and they are given by

$$
F_{0}(u)=\frac{1}{2 \lambda_{\mathrm{GB}}}\left(1-\sqrt{1-4 \lambda_{\mathrm{GB}}\left(1-\frac{u^{4}}{u_{\mathrm{H}}^{4}}\right)}\right), \quad B_{0}=\frac{1+\sqrt{1-4 \lambda_{\mathrm{GB}}}}{2} .
$$

The boundary conditions were fixed such that $F$ vanishes at the horizon $u=u_{\mathrm{H}}$. At the boundary $u=0$ we have

$$
\phi_{2, \mathrm{bdry}}=0, \quad F_{2, \mathrm{bdry}}=0, \quad B_{2, \mathrm{bdry}}=0, \quad H_{2, \mathrm{bdry}}=0 .
$$

The solution is regular everywhere and asymptotically approaches $A d S_{5}$. Thermodynamical quantities such as temperature and entropy can be computed via standard formulas, by requiring the regularity of the (Euclideanized) metric at the horizon and using the area 
law, respectively. One finds

$$
\begin{aligned}
& T=\sqrt{B_{0}}\left(\frac{1}{\pi u_{\mathrm{H}}}-\frac{2 B_{0}-6 \lambda_{\mathrm{GB}}+\sqrt{\lambda_{\mathrm{GB}}} \log \left(\frac{1+2 \sqrt{\lambda_{\mathrm{GB}}}}{1-2 \sqrt{\lambda_{\mathrm{GB}}}}\right)-\log \left(\frac{4 B_{0}}{\sqrt{1-4 \lambda_{\mathrm{GB}}}}\right)}{48 \pi\left(1-4 \lambda_{\mathrm{GB}}\right)} u_{\mathrm{H}} a^{2}+O\left(a^{4}\right)\right) . \\
& s=\frac{\pi}{4 G B_{0}^{3 / 2}}\left(\pi^{2} T^{3}+\frac{1}{8} T B_{0} a^{2}+O\left(a^{4}\right)\right) .
\end{aligned}
$$

\subsection{Shear viscosity}

An important quantity to compute in a plasma is the ratio of shear viscosity over entropy density. ${ }^{1}$ This is a rather universal quantity for theories with an Einstein dual, which has been conjectured to obey the Kovtun-Son-Starinets (KSS) bound $\eta / s \geq 1 / 4 \pi$ [76]. This bound can however be violated by the inclusion of higher derivative corrections [97] (see also [99-103, 115-119]) and by the breaking of spatial isotropy [29, 30]; see [104] for a status report on the viscosity bound.

In this section, we employ the membrane paradigm, proposed in [120] and used in [29] for the anisotropic plasma of [22], to compute $\eta / s$ for our geometry (6.2). ${ }^{2}$ Appendix $\mathrm{C}$ contains an alternative derivation of the result in this section using the Kubo formula. As in [29], we will be interested in two components of the shear viscosity tensor: $\eta_{x y x y}$, which is entirely in the transverse (isotropic) plane, and $\eta_{x z x z}=\eta_{y z y z}$, which mixes the anisotropic direction $z$ with one of the directions in the transverse plane. We denote these two components as

$$
\eta_{\perp}=\eta_{x y x y}, \quad \eta_{\|}=\eta_{x z x z}
$$

To calculate these viscosities we consider the fluctuations $h_{x y}$ and $h_{x z}$ around the background (6.2). Given the symmetry in the transverse plane, we can take these fluctuations to depend solely on $(t, y, z, u)$. The equations of motion for $\psi_{\perp}=h_{y}^{x}(t, y, z, u)$ and $\psi_{\|}=h_{z}^{x}(t, y, z, u)$ decouple from all other equations and from each other. In both cases, they have the following form

$$
a(u) \psi^{\prime \prime}+b(u) \psi^{\prime}+c(u) \psi=0,
$$

where $a(u), b(u)$ and $c(u)$ are functions of the background fields and $\psi$ stands for either $\psi_{\perp}$ or $\psi_{\|}$, depending on the case. Here the primes denote derivatives with respect to $u$. To use the membrane paradigm, we need to write an effective action for $\psi_{\perp}$ and $\psi_{\|}$. To

\footnotetext{
${ }^{1}$ Other observables that have been computed in Einstein plus GB gravity can be found in, e.g., [54,114].

${ }^{2}$ The computation of the shear viscosity in an anisotropic superfluid with a GB term has recently been presented in [121].
} 
this scope we write $(6.7)$ in the form ${ }^{3}$

$$
\left(n(u) \psi^{\prime}\right)^{\prime}-m(u) \psi=0
$$

with

$$
n(u)=\exp \left(\int_{u} d u^{\prime} \frac{a\left(u^{\prime}\right)}{b\left(u^{\prime}\right)}\right), \quad m(u)=\frac{c(u)}{a(u)} \exp \left(\int_{u} d u^{\prime} \frac{a\left(u^{\prime}\right)}{b(u)}\right) .
$$

The effective action that gives rise to the equation of motion above is

$$
S_{\mathrm{eff}}=-\frac{1}{2} \frac{1}{16 \pi G} \int d^{4} x d u\left[n(u)\left(\psi^{\prime}\right)^{2}-m(u) \psi^{2}\right] \text {. }
$$

To compare this action with the one of [120], we need to transform it to Fourier space. To do that, we write

$$
\psi(t, y, z, u)=\int \frac{d \omega}{2 \pi} \frac{d^{3} k}{(2 \pi)^{3}} \psi(u) e^{-i \omega t+i k_{y} y+i k_{z} z},
$$

where we have used the axial symmetry to rotate $\mathbf{k}=\left(0, k_{y}, k_{z}\right)$. Plugging (6.11) into (6.10) and using Plancherel's theorem, it can be shown that

$$
S_{\mathrm{eff}}=-\frac{1}{2} \frac{1}{16 \pi G} \int \frac{d \omega}{2 \pi} \frac{d^{3} k}{(2 \pi)^{3}} d u\left[n(u)\left(\psi^{\prime}\right)^{2}-m(u) \psi^{2}\right]
$$

Using the notation of [120], this can be recast in the following form

$$
S_{\text {eff }}=-\frac{1}{2} \int \frac{d \omega}{2 \pi} \frac{d^{3} k}{(2 \pi)^{3}} d u \sqrt{-g}\left[\frac{g^{u u}}{Q(u, k)}\left(\psi^{\prime}\right)^{2}+P(u, k) \psi^{2}\right],
$$

with

$$
\frac{1}{16 \pi G} n(u)=\frac{\sqrt{-g} g^{u u}}{Q(u, k)} .
$$

The shear viscosity is then obtained as [120]

$$
\frac{\eta}{s}=\frac{1}{4 \pi} \frac{16 \pi G}{Q\left(u_{\mathrm{H}}, k \rightarrow 0\right)}
$$

Writing the equations of motion for $\psi_{\perp}$ and $\psi_{\|}$, we can obtain explicit expressions for the $n(u)$ 's and $m(u)$ 's. Putting these together with (6.14) and (6.15), it is readily found that

$$
\frac{\eta_{\perp}}{s}=\frac{1}{4 \pi}\left(\frac{g_{x x}}{g_{y y}}-\frac{\lambda_{\mathrm{GB}}}{2} \frac{g_{x x}^{2} g_{t t}^{\prime} g_{z z}^{\prime}}{g}\right)
$$

\footnotetext{
${ }^{3}$ It is important to emphasize that $n(u)$ and $m(u)$ are not the same in the equations of motion for $\psi_{\perp}$ and $\psi_{\|}$. Here $n(u)$ stands for either $n_{\perp}$ or $n_{\|}$, and $m(u)$ stands for either $m_{\perp}$ or $m_{\|}$.
} 


$$
\frac{\eta_{\|}}{s}=\frac{1}{4 \pi}\left(\frac{g_{x x}}{g_{z z}}-\frac{\lambda_{\mathrm{GB}}}{2} \frac{g_{x x}^{2} g_{t t}^{\prime} g_{y y}^{\prime}}{g}\right)
$$

These results are completely generic for the system we have considered. In particular, we can check them against the known results from pure Einstein-Hilbert gravity with a GB term [97] and with the anisotropic background of [29], finding perfect agreement in both cases. In the first case, we need to take the limit of $a \rightarrow 0$ of the equations above. We find

$$
\frac{\eta_{\perp}}{s}=\frac{\eta_{\|}}{s}=\frac{1-4 \lambda_{\mathrm{GB}}}{4 \pi},
$$

as in [97]. To perform the second check we take the limit $\lambda_{\mathrm{GB}} \rightarrow 0$ and obtain ${ }^{4}$

$$
\frac{\eta_{\perp}}{s}=\frac{1}{4 \pi}, \quad \frac{\eta_{\|}}{s}=\frac{1}{4 \pi} \frac{1}{H\left(u_{\mathrm{H}}\right)}=\frac{1}{4 \pi}-\frac{\log 2}{16 \pi^{3}}\left(\frac{a}{T}\right)^{2}+O\left(a^{4}\right) .
$$

Note how the longitudinal shear viscosity violates the KSS bound.

Specializing (6.16) to our solution (6.2) we find

$$
\begin{aligned}
\frac{\eta_{\perp}}{s} & =\frac{1-4 \lambda_{\mathrm{GB}}}{4 \pi}+\frac{B_{0}}{24 \pi^{3}} \frac{\lambda_{\mathrm{GB}}\left(3-4 \lambda_{\mathrm{GB}}\right)}{\left(1-4 \lambda_{\mathrm{GB}}\right)}\left(\frac{a}{T}\right)^{2}+O\left(a^{4}\right), \\
\frac{\eta_{\|}}{s} & =\frac{1-4 \lambda_{\mathrm{GB}}}{4 \pi}+\frac{B_{0}}{32 \pi^{3}} G\left(\lambda_{\mathrm{GB}}\right)\left(\frac{a}{T}\right)^{2}+O\left(a^{4}\right)
\end{aligned}
$$

where $G\left(\lambda_{\mathrm{GB}}\right)$ is given by

$$
\begin{aligned}
G\left(\lambda_{\mathrm{GB}}\right)=-1+ & 2 \lambda_{\mathrm{GB}}\left(\frac{8 \lambda_{\mathrm{GB}}}{12 \lambda_{\mathrm{GB}}-3}+1\right)+\sqrt{1-4 \lambda_{\mathrm{GB}}} \\
& +\sqrt{\lambda_{\mathrm{GB}}} \log \left(\frac{1+2 \sqrt{\lambda_{\mathrm{GB}}}}{1-2 \sqrt{\lambda_{\mathrm{GB}}}}\right)+\log \left(\frac{\sqrt{1-4 \lambda_{\mathrm{GB}}}-1+4 \lambda_{\mathrm{GB}}}{8 \lambda_{\mathrm{GB}}}\right)
\end{aligned}
$$

We emphasize that these results, despite being of second order in $a$, are fully nonperturbative in $\lambda_{\mathrm{GB}}$. The KSS bound might be violated in this setting both by the anisotropy and by the GB coupling.

\subsection{Drag force}

When a heavy quark propagates through a strongly coupled plasma it loses energy due to the interaction with the medium. One quantity related to the dissipation of energy of the quark is the drag force. The study of drag force in a strongly coupled plasma was initiated in $[122,123]$ for the case of (isotropic) $\mathcal{N}=4 \mathrm{SYM}$ and subsequently it was

\footnotetext{
${ }^{4}$ Note that to compare the expressions for $\eta_{\|}$one needs to take into account the different factors of the dilaton in the Ansäzte of $[22,29]$ and (6.1).
} 
extended in several ways. See, for instance, [32,114,124-129]. The two computations of the drag force closely related to the present work were done in $[31,130]$, corresponding to the limits $\lambda_{\mathrm{GB}}=0$ and $a=0$, respectively.

Following the standard prescription of the computation of the drag force, we consider an external heavy quark moving through the strongly coupled plasma with constant velocity $v$. Since the heavy quark loses energy due to the interaction with the plasma, an external force is necessary to maintain the motion stationary. In the dual picture, we have a classical string with an endpoint in the quark (at the boundary) and the other endpoint in the bulk, in a picture usually referred to as "trailing string" [122, 123]. The derivation of the general formula is presented in Appendix D. As a result, we first need to determine a critical point $u_{c}$ by solving the equation

$$
\left[\frac{2 G_{t t}}{v^{2}}+G_{x x}+G_{z z}+\left(G_{z z}-G_{x x}\right) \cos (2 \varphi)\right]_{u=u_{c}}=0
$$

where $\varphi$ is the angle between the direction of motion of the quark and the $z$-direction. In what follows, we will be interested in the cases where the motion of the quark is parallel (\|) and transversal $(\perp)$ to the direction of anisotropy, corresponding to $\varphi=0$ and $\varphi=\pi / 2$, respectively. Once the critical point is determined, it is straightforward to compute the drag force using

$$
F_{\text {drag }}^{\|}=\left.e^{\phi / 2} G_{z z} v\right|_{u=u_{c}}, \quad F_{\text {drag }}^{\perp}=\left.e^{\phi / 2} G_{x x} v\right|_{u=u_{c}} .
$$

Since we are working in the small anisotropy regime, the critical point can be written as

$$
u_{c}=u_{0 c}+a^{2} u_{2 c}+O\left(a^{4}\right) .
$$

For our particular background, the equation for the critical point (6.21) expanded to second order in $a$ becomes

$$
B_{0} F_{0}-v^{2}+\left.a^{2}\left(B_{0} B_{2} F_{0}+B_{0} F_{2}-v^{2} \cos ^{2} \varphi H_{2}+B_{0} u_{2 c} F_{0}^{\prime}\right)\right|_{u_{0_{c}}}=0
$$

Solving the equation order by order gives

$$
\begin{aligned}
& u_{0 c}=u_{\mathrm{H}}\left(\frac{B_{0}^{2}-v^{2} B_{0}+v^{4} \lambda_{\mathrm{GB}}}{B_{0}^{2}}\right)^{\frac{1}{4}}, \\
& u_{2 c}=-\frac{B_{0} B_{2}\left(u_{0 c}\right) F_{0}\left(u_{0 c}\right)+B_{0} F_{2}\left(u_{0 c}\right)-v^{2} H_{2}\left(u_{0 c}\right) \cos ^{2} \varphi}{B_{0} F_{0}^{\prime}\left(u_{0 c}\right)} .
\end{aligned}
$$

Plugging the solution for the critical point (6.25) into the formulas of the drag force (6.22), 
we obtain

$$
\begin{aligned}
& F_{\text {drag }}^{\|}=\frac{v}{u_{0 c}^{2}}+\frac{a^{2} v}{2 u_{0 c}^{2}}\left(\phi_{2}\left(u_{0 c}\right)-4 \frac{u_{2 c}}{u_{0 c}}+2 H_{2}\left(u_{0 c}\right)\right)+O\left(a^{4}\right), \\
& F_{\text {drag }}^{\perp}=\frac{v}{u_{0 c}^{2}}+\frac{a^{2} v}{2 u_{0 c}^{2}}\left(\phi_{2}\left(u_{0 c}\right)-4 \frac{u_{2 c}}{u_{0 c}}\right)+O\left(a^{4}\right) .
\end{aligned}
$$

We do not report the full explicit expressions for the drag force here since they are too long and not very illuminating. Since $T$ is a function of $u_{\mathrm{H}}$, we can invert this relation to express the drag force as a function of the temperature. ${ }^{5}$ We can then check that in the limit $\lambda_{\mathrm{GB}} \rightarrow 0$ we recover the result of [31],

$$
\begin{aligned}
F_{\mathrm{drag}}^{\| \mathrm{MT}} & =\frac{\pi^{2} T^{2} v}{\sqrt{1-v^{2}}}+\frac{a^{2} v\left(-v^{2}+\sqrt{1-v^{2}}+\left(v^{2}+1\right) \log \left(\sqrt{1-v^{2}}+1\right)+1\right)}{24\left(1-v^{2}\right)^{3 / 2}} \\
F_{\mathrm{drag}}^{\perp \mathrm{MT}} & =\frac{\pi^{2} T^{2} v}{\sqrt{1-v^{2}}}+\frac{a^{2} v\left(-v^{2}+\sqrt{1-v^{2}}+\left(4 v^{2}-5\right) \log \left(\sqrt{1-v^{2}}+1\right)+1\right)}{24\left(1-v^{2}\right)^{3 / 2}}
\end{aligned}
$$

and in the limit $a \rightarrow 0$ we recover the result of [130]

$$
F_{\mathrm{drag}}^{\mathrm{GB}}=\frac{\sqrt{2} \pi^{2} T^{2} v}{\sqrt{\left(v^{2}-1\right)\left(2\left(v^{2}+1\right) \lambda_{\mathrm{GB}}-\sqrt{1-4 \lambda_{\mathrm{GB}}}-1\right)}} .
$$

Of course, in the limit where both $a$ and $\lambda_{\text {GB }}$ go to zero we recover the isotropic $\mathcal{N}=4$ SYM result $[122,123]$

$$
F_{\text {drag }}^{\text {iso }}=\frac{\pi^{2} T^{2} v}{\sqrt{1-v^{2}}} .
$$

In the analysis of our results, it is useful to normalize the drag force by the isotropic result (6.29). The normalized drag force here depends on $v, \lambda_{\mathrm{GB}}$ and $a / T$. The main result is shown in Figure 6.1. Our results are, as expected, a combined effect of their limiting cases $[31,130]$. The effect of the Gauss-Bonnet coupling is, in general, to enhance the drag force for $\lambda_{\mathrm{GB}}>0$ and to decrease it for $\lambda_{\mathrm{GB}}<0$, for both longitudinal and transversal motion. This is the same effect observed in the case of pure Gauss-Bonnet gravity [130], but it is different from what happens with corrections of type $\alpha^{\prime 3} R^{4}$, where the drag force is always enhanced [128]. The effect of the anisotropy is qualitatively the same found in [31]: for the transversal motion the drag force can increase or decrease, while for the parallel motion the drag force increases in general (except for sufficiently large negative values of $\lambda_{\mathrm{GB}}$ ). We also plotted the drag force as a function of the quark velocity (Figure 6.2). In general, the drag is increased for larger velocities and there is a divergence in the

\footnotetext{
${ }^{5}$ The easiest way to write the drag force in terms of the temperature is by noting that the critical point scales as $u_{c}=u_{\mathrm{H}} \gamma_{0}+a^{2} u_{\mathrm{H}}^{3} \gamma_{2}+O\left(a^{4}\right)$, where $\gamma_{0}$ and $\gamma_{2}$ are quantities that do not depend on $u_{\mathrm{H}}$.
} 
limit $v \rightarrow 1$, similarly to what occurred in [31].
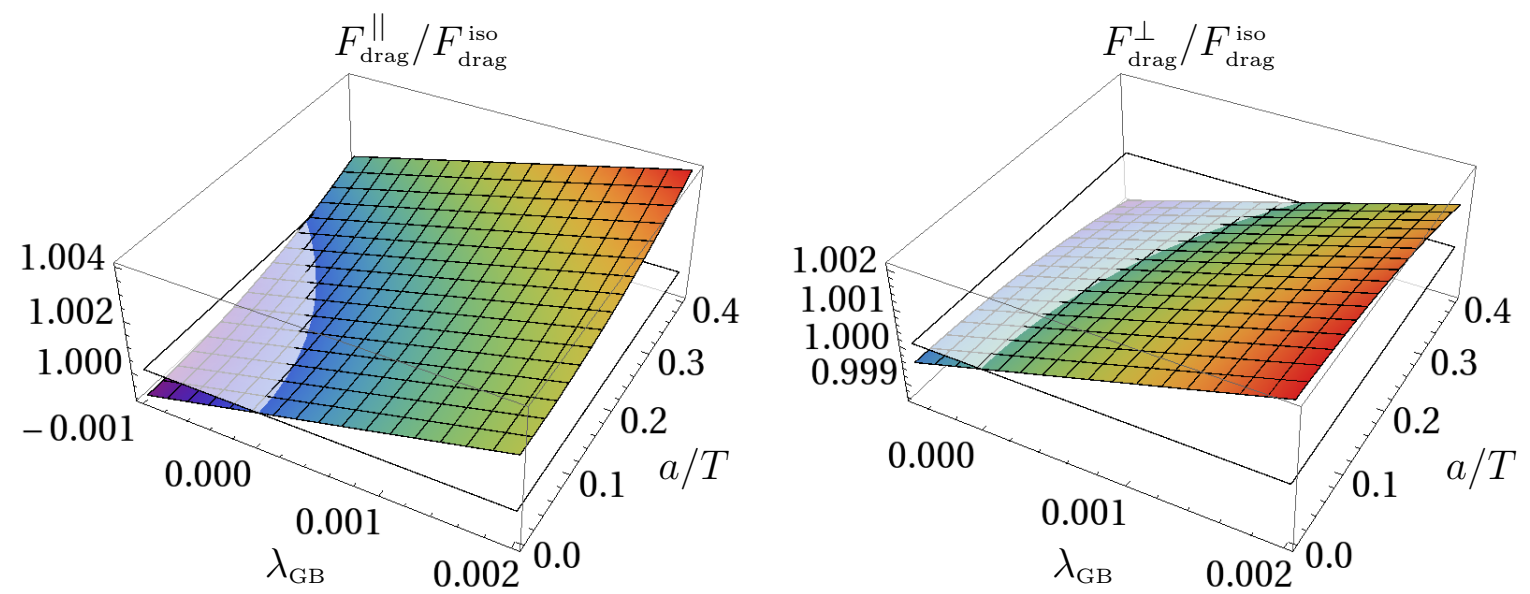

Figure 6.1: Drag force normalized by the isotropic result as a function of $\left(\lambda_{G B}, \frac{a}{T}\right)$. Here we have fixed $v=0.3$. Left: Motion along the anisotropic direction. Right: Motion along the direction transversal to the anisotropy.
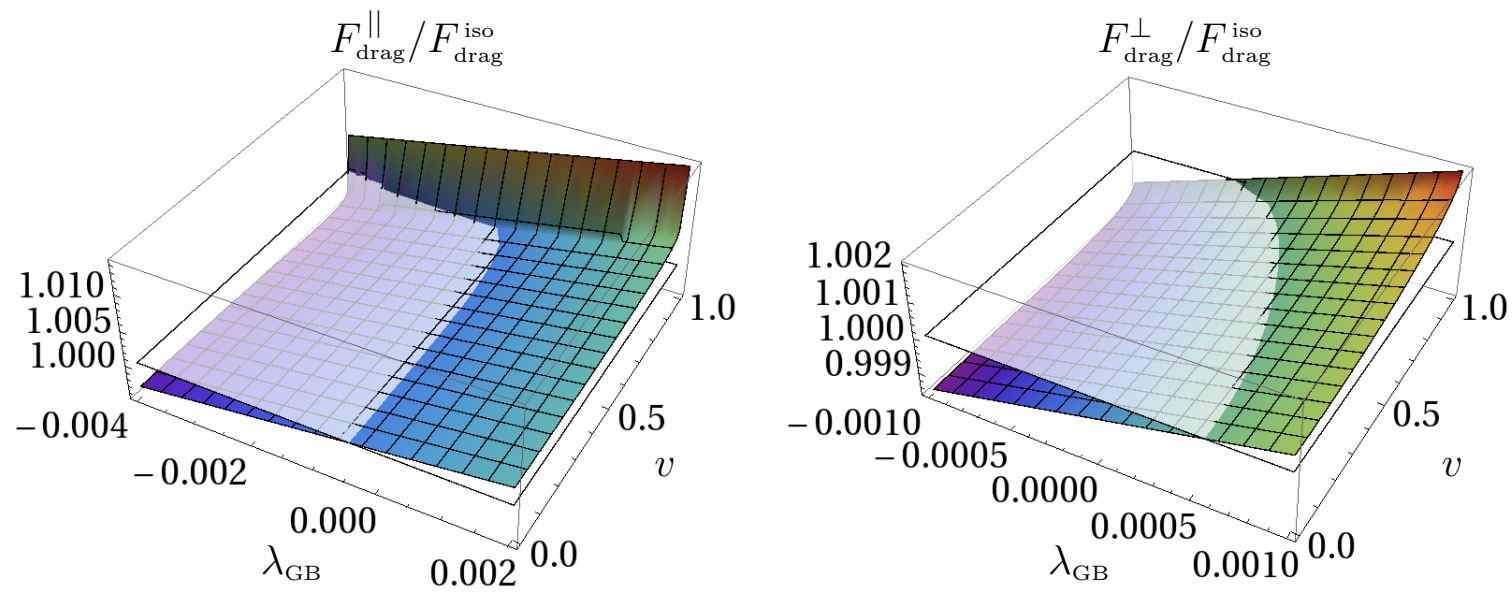

Figure 6.2: Drag force normalized by the isotropic result as a function of $\left(\lambda_{G B}, v\right)$. Here we have fixed $\frac{a}{T}=0.2$. Left: Motion along the anisotropic direction. Right: Motion along the direction transversal to the anisotropy. For other values of $\frac{a}{T}$ the results were qualitatively the same.

\subsection{Jet quenching parameter}

Results from RHIC [131-134] indicate a strong suppression of particles with high transversal momentum $p_{\mathrm{T}}$ in $\mathrm{Au}-\mathrm{Au}$ collisions, but not in $\mathrm{d}-\mathrm{Au}$ collisions. The explana- 
tion for this phenomenon is that in $\mathrm{Au}-\mathrm{Au}$ collisions the hot and dense quark gluon plasma is produced, and the jets lose energy due to the interaction with this medium before hadronising. This energy loss effect is called "jet quenching" and can give valuable information about the properties of the plasma. One important quantity related to jet quenching is the jet quenching parameter $\hat{q}$, which quantify the change of transverse momentum of the parton per unit length when suffering multiple scattering with the medium. The change in transverse momentum is usually referred to as "momentum broadening".

The jet quenching parameter has been calculated at weak coupling for several models (see [135] for a review) and has been consistent with data [136]. However, the assumption of weak coupling is still not well justified, since different energy scales are involved in heavy ion collision experiments and thus a calculation at strongly coupling may be necessary. This motivates a non-perturbative definition of the jet quenching parameter. The nonperturbative definition of the jet quenching parameter and its first computation using holography was done in [137-139]. After that, it was extended in several directions. ${ }^{6}$ See, for instance, [141-143].

The non-perturbative definition of the jet quenching parameter $\hat{q}$ was inspired by its perturbative calculation in the so called dipole approximation [144]

$$
\left\langle W^{A}(\mathcal{C})\right\rangle \simeq \exp \left[-\frac{L^{-} \ell^{2}}{4 \sqrt{2}} \hat{q}\right]
$$

where $W^{A}(\mathcal{C})$ is a rectangular light-like Wilson loop in the adjoint representation with sizes $L^{-}$and $\ell$, with $L^{-} \gg \ell$. Using the holographic dictionary the jet quenching parameter is given in terms of the on-shell Nambu-Goto action whose string worldsheet boundary coincides with the Wilson loop ${ }^{7}$

$$
\hat{q}=\frac{8 \sqrt{2}}{L^{-} \ell^{2}} S^{\text {on-shell }}
$$

In the case of pure (isotropic) $\mathcal{N}=4 \mathrm{SYM}$, the result obtained was [137-139]

$$
\hat{q}_{\text {iso }}=\frac{\pi^{3 / 2} \Gamma\left(\frac{3}{4}\right)}{\Gamma\left(\frac{5}{4}\right)} \sqrt{\lambda} T^{3} .
$$

Here we compute the jet quenching parameter for the anisotropic background with Gauss-Bonnet term. A detailed derivation of the formula we used here is presented in Appendix E. The parameters involved are the Gauss-Bonnet coupling $\lambda_{\mathrm{GB}}$, the ratio of

\footnotetext{
${ }^{6}$ There are also some attempts of non-perturbative computations of the jet quenching parameter on the lattice (see, for instance, [140]).

${ }^{7}$ The extra factor of 2 comes from the fact that, for large $N_{c}$, the Wilson loop in the adjoint representation is roughly speaking the square of the Wilson loop in the fundamental representation.
} 
the anisotropy parameter to temperature $a / T$ and the angles $(\theta, \varphi)$ associated with the direction of motion of the quark and the direction of the momentum broadening, respectively. ${ }^{8}$

Our results are summarized in Figure 6.3. Similarly to the drag force computation of the previous subsection, the effect of the Gauss-Bonnet coupling is controlled by its sign: the jet quenching parameter is increased for $\lambda_{\mathrm{GB}}>0$ and decreased for $\lambda_{\mathrm{GB}}<0$. The effect of the anisotropy, in the small anisotropy limit, is to increase the jet quenching parameter as it occurred in $[32,37,51]$, with the highest increase taking place when the quark moves in the anisotropic direction. We also verified that, for a fixed value of $\theta$, the jet quenching parameter is slightly larger for the momentum broadening taking place in the anisotropic $(\varphi=\pi / 2)$ direction than in the transversal direction to the anisotropy $(\varphi=0)$.
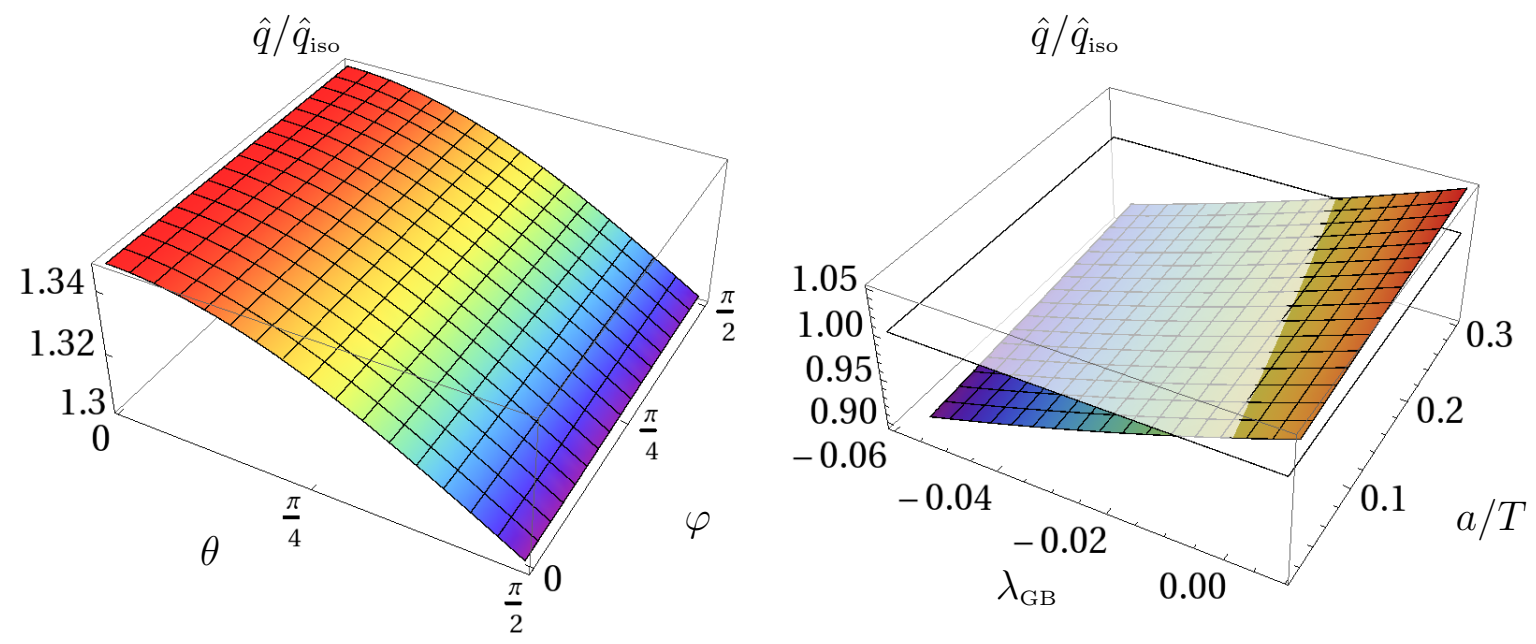

Figure 6.3: Left: Jet quenching parameter as a function of $(\theta, \varphi)$. We have set $\lambda_{G B}=0.1$ and $a / T=0.33$. Right: The jet quenching parameter as a function of $\left(\lambda_{G B}, \frac{a}{T}\right)$. We have set $\theta=\varphi=\pi / 4$. Both plots were normalized by the isotropic result (6.32).

As argued in [136], weak coupling models of jet quenching are in general lower than the value obtained at strong coupling for $\mathcal{N}=4 \mathrm{SYM}$ (6.32). If we were expecting a smooth interpolation between the weak and strong coupling values, the case $\lambda_{\mathrm{GB}}<0$ becomes particularly interesting since it decreases the $\mathcal{N}=4 \mathrm{SYM}$ result. The same decreasing effect was also found in [145], where they considered fluctuations of the string worldsheet, and in [146], where curvature corrections of type $\alpha^{\prime 3} R^{4}$ in the AdS-Schwarzschild background

\footnotetext{
${ }^{8}$ More precisely, $\theta$ is the angle between the direction of motion of the quark and the anisotropic direction. The direction of the momentum broadening is transversal to the direction of motion of the quark and forms an angle $\varphi$ with the $y$-axis. Note that the same symbols $\theta$ and $\varphi$ were used for other observables, but with different meanings.
} 
were taken into account.

\subsection{Quarkonium static potential}

Quarkonium mesons are produced in the early stages of heavy ion collisions, before the creation of the QGP. As they are much more tightly bound and smaller than ordinary 'light' hadrons, they can survive as bound states in the QGP at temperatures above the deconfinement temperature up to some dissociation temperature. This property, together with the fact that their interaction with the thermal medium is comparatively stronger than their interaction with the hadronic matter formed after hadronization, makes the quarkonium mesons excellent probes to study the QGP formed in heavy ion collisions [147].

Here we study the static quarkonium potential in a strongly coupled plasma dual to the anisotropic gravity theory with the Gauss-Bonnet term. In particular, we analyze how the anisotropy and the higher derivative terms affect the potential energy and the screening length of a heavy quark-antiquark pair. The holographic studies of this quantity were initiated in $[148,149]$, for infinitely heavy quarks in the $\mathcal{N}=4$ SYM theory and, since then, several extensions of this work have been performed. See, for instance, [38,150-159]. Higher derivative corrections to the quarkonium potential were considered in $[154,159]$ and the effects of anisotropy were taken into account in $[32,38]$.

The static quarkonium potential can be extracted from the expectation value of a Wilson loop

$$
\lim _{\mathcal{T} \rightarrow \infty}\langle W(\mathcal{C})\rangle \sim e^{i \mathcal{T}\left(V_{Q \bar{Q}}+2 M_{Q}\right)},
$$

where $\mathcal{C}$ is a rectangular loop with time extension $\mathcal{T}$ and spatial extension $L, V_{Q \bar{Q}}$ is the quark-antiquark potential energy and $M_{Q}$ is the quark mass. The Wilson loop can be viewed as a static quark-antiquark pair separated by a distance $L$. In the gravity side, the pair is described by an open string with both endpoints attached to a D7-brane sitting at some AdS radial position which determines the quark mass $\left(M_{Q} \sim 1 / u\right)$. For simplicity, we will work in the case where the D7-brane is at the boundary of AdS and, consequently, the quark is infinitely heavy and non-dynamical.

In the large $N_{c}$ and large $\lambda$ limits the Wilson loop of Eq. (6.33) can be calculated in the gravity side by the expression

$$
\lim _{\mathcal{T} \rightarrow \infty}\langle W(\mathcal{C})\rangle=e^{i S^{(\text {on-shell })}}
$$

where $S^{\text {(on-shell) }}$ is the on-shell Nambu-Goto action of a U-shaped string whose worldsheet 
boundary coincides with the curve $\mathcal{C}$. The quarkonium potential is thus obtained as

$$
V_{Q \bar{Q}}=\frac{S^{(\text {on-shell })}}{\mathcal{T}}-2 M_{Q}
$$

where the quark mass $M_{Q}$ is determined by evaluating the Nambu-Goto action of a straight string connecting the boundary to the horizon. Given the rotational symmetry in the $x y$-plane, we can assume the quark-antiquark pair to lie in the $x z$-plane, forming an angle $\theta$ with the $z$-direction. Since we want to focus on the results, we leave the details of the calculation of $V_{Q \bar{Q}}(L)$ in Appendix F.

First, let us discuss some general properties of $V_{Q \bar{Q}}(L)$. From Figure 6.4, we see that $V_{Q \bar{Q}}$ only exists up to a maximum separation length $L_{\max }$. For each value of $L \leq L_{\max }$ there are two possible string configurations corresponding to the upper and lower parts of $V_{Q \bar{Q}}$. It turns out that only the lower part of $V_{Q \bar{Q}}$ represents a physical solution [150]. Note that $V_{Q \bar{Q}}=0$ at some point $L=L_{s}$, usually referred to as "screening length". Since $V_{Q \bar{Q}}$ represents the difference between the energy and mass of the quarkonium, a negative value of the potential $\left(L \leq L_{s}\right.$ ) represents a situation where the U-shaped string (bound state) is energetically favorable over the configuration with two straight strings (unbound state). On the other hand, when the potential is positive $\left(L \geq L_{s}\right)$, the opposite happens and the unbound configuration is energetically favorable. ${ }^{9}$ Another point is that the screening of a plasma is weaker for large $L_{s}$ and stronger for small $L_{s}$. This is because $L_{s}$ represents the separation in which the interaction between the quark and the antiquark becomes completely screened by the medium.

\footnotetext{
${ }^{9}$ However, we emphasize that the solution for $V_{Q \bar{Q}}$ is not valid when $L \geq L_{s}$. In this case the quarkantiquark interaction is completely screened by the presence of QGP between them and, as a consequence, their separation can be increased with no additional energy cost. This implies that the potential is actually constant for $L \geq L_{s}$. The dual gravity picture can be understood as follows: as we increase the quarkantiquark separation, the U-shaped string connecting the quarks eventually touches the horizon. At this point the string can minimize its energy by splitting into straight strings connecting the boundary of AdS to the horizon.
} 


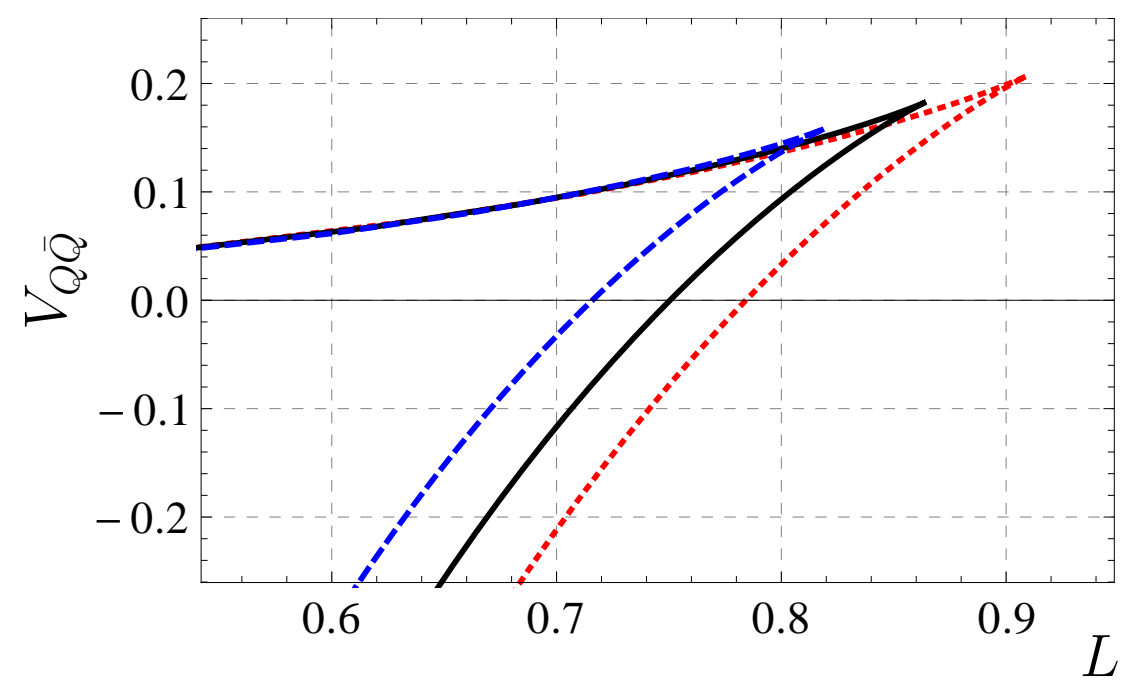

Figure 6.4: Quark-antiquark potential $V_{Q \bar{Q}}$ as a function of their separation $L$ for different values of the Gauss-Bonnet coupling: $\lambda_{G B}=-0.1$ (red, dotted), $\lambda_{G B}=0$ (black, solid) and $\lambda_{G B}=0.1$ (blue, dashed). For all curves $a / T \approx 0.3$ and $\theta=\pi / 4$.

Figure 6.4 shows that positive values of $\lambda_{\mathrm{GB}}$ decrease the screening length, while negative values of $\lambda_{\mathrm{GB}}$ increase this quantity. This effect can be better visualized in Figure 6.5 (a), where the screening length is presented as a function of $\left(\lambda_{\mathrm{GB}}, a\right)$. Now let us discuss the effect of the anisotropy. Firstly, Figure 6.5 (b) shows that the screening length for a quarkonium oriented along the anisotropic direction $(\theta=0)$ is always smaller than the corresponding quantity for a quarkonium oriented in the transverse plane $(\theta=\pi / 2)$. Secondly, the 2D plot of Figure 6.6 reveals that the screening length always decrease as we increase $a / T$, for any orientation of the pair, at fixed $\lambda_{\mathrm{GB}}$. These anisotropic effects are also observed in holographic calculations at strong coupling when the anisotropy is introduced by a magnetic field [158] and at weak coupling in calculations based on "hardthermal-loop" resummed perturbative QCD [160]. The limit $\lambda_{\mathrm{GB}} \rightarrow 0$ of the above results agrees with the calculations of [32]. We also checked that the limit $a \rightarrow 0$ for $V_{Q \bar{Q}}$ agrees with the results of [159] when the Quasi-topological coupling constant is zero. ${ }^{10}$

\footnotetext{
${ }^{10}$ In the comparison of our results with [159], one should note that the potential of [159] is normalized with $1 /\left(\pi \alpha^{\prime}\right)$, while our results are normalized with $1 /\left(2 \pi \alpha^{\prime}\right)$.
} 


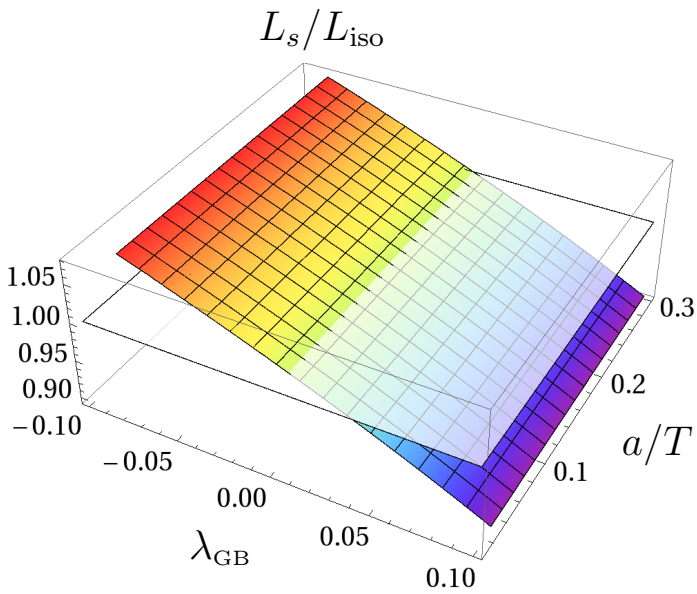

(a)

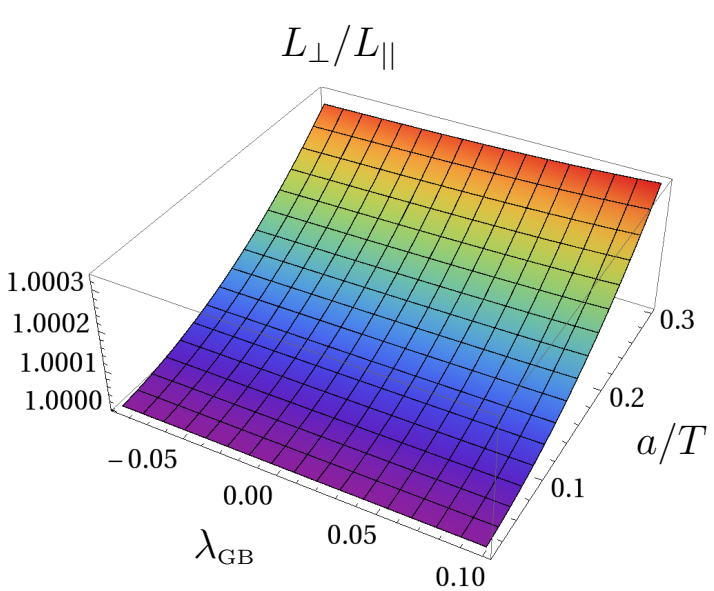

(b)

Figure 6.5: (a) Screening length $L_{s}\left(\lambda_{G B}, a\right)$ normalized with respect to the isotropic result $L_{\text {iso }}=$ $L_{s}\left(\lambda_{G B}=0, a=0\right)$ for $\theta=0$. (b) Ratio $L_{\perp} / L_{\|}$, where $L_{\perp}$ is the screening length calculated at $\theta=\pi / 2$, and $L_{\|}$is the screening length calculated at $\theta=0$.

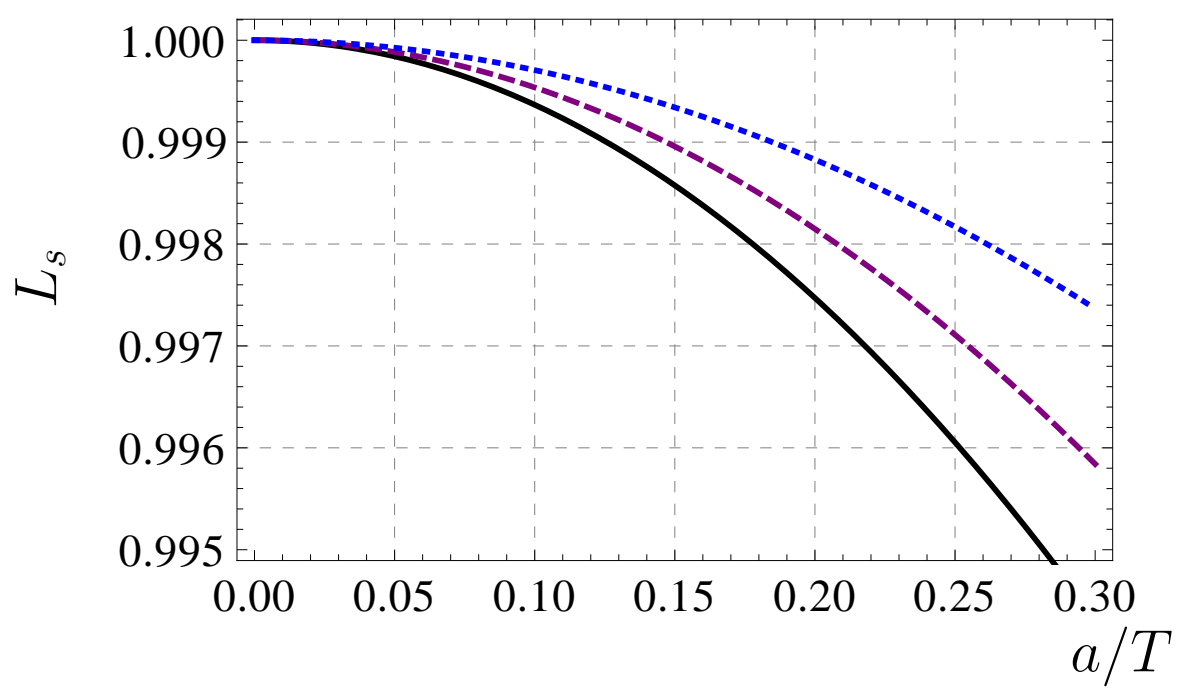

Figure 6.6: Screening length $L_{s}$ as a function of a $/ T$ for three different quarkonium orientations: $\theta=0$ (black, solid), $\theta=\pi / 4$ (purple, dashed) and $\theta=\pi / 2$ (blue, dotted). The Gauss-Bonnet coupling is fixed $\lambda_{G B}=0$.

\subsection{Photon production}

The limited extension of the QGP created in heavy ion collisions and the weakness of the electromagnetic interactions imply that this medium should be optically thin. Therefore, the photons produced in the plasma escape from it without subsequent interactions, providing an excellent probe of the conditions of the medium. The holographic stud- 
ies of this quantity were initiated in [161] and extended in several directions, see, for instance $[45,46,48,162-172]$. In this section we study how the anisotropy and higher derivative corrections affect the photon production rate.

Let $\mathcal{L}_{0}$ be the Lagrangian of the field theory dual to the gravity theory described by the action (5.1). The photon production rate is calculated by adding a dynamical photon to $\mathcal{L}_{0}$ coupled to the electric charged matter fields, that is

$$
\mathcal{L}=\mathcal{L}_{0}+e J_{\mu}^{\mathrm{EM}} A^{\mu}-\frac{1}{4} F_{\mu \nu} F^{\mu \nu},
$$

where $F_{\mu \nu}=\partial_{\mu} A_{\nu}-\partial_{\nu} A_{\mu}$ is the field strength associated to the photon field $A^{\mu}, e$ is the electromagnetic coupling constant and $J_{\mu}^{\mathrm{EM}}$ is the electromagnetic current. At leading order in $e$, the number of photons emitted per unit time and unit volume is given by [173]

$$
\frac{d \Gamma_{\gamma}}{d^{3} k}=\left.\frac{e^{2}}{(2 \pi)^{3} 2|\vec{k}|} \Phi(k) \eta^{\mu \nu} \chi_{\mu \nu}(k)\right|_{k^{0}=|\vec{k}|}
$$

where $\eta^{\mu \nu}=\operatorname{diag}(-+++)$ is the Minkowski metric, $k^{\mu}=\left(k^{0}, \vec{k}\right)$ is the photon null momentum, $\Phi(k)$ is the distribution function and $\chi_{\mu \nu}$ is the spectral density. Assuming thermal equilibrium, the distribution function reduces to the Bose-Einstein distribution $n_{B}\left(k^{0}\right)=1 /\left(e^{k^{0} / T}-1\right)$. The spectral density can be obtained as

$$
\chi_{\mu \nu}(k)=-2 \operatorname{Im} G_{\mu \nu}^{\mathrm{R}}(k),
$$

where

$$
G_{\mu \nu}^{\mathrm{R}}(k)=-i \int d^{4} x e^{-i k \cdot x} \Theta(t)\left\langle\left[J_{\mu}^{\mathrm{EM}}(x), J_{\nu}^{\mathrm{EM}}(0)\right]\right\rangle
$$

is the retarded correlator of two electromagnetic currents $J_{\mu}^{\mathrm{EM}}$ and the above expectation value is taken in the thermal equilibrium state. The Ward identity $k^{\mu} \chi_{\mu \nu}=0$ for null $k^{\mu}$ implies that only the transverse spectral functions contribute in the calculation of the trace of the spectral density, that is,

$$
\eta^{\mu \nu} \chi_{\mu \nu}=\left.\sum_{s=1,2} \epsilon_{(s)}^{\mu}(\vec{k}) \epsilon_{(s)}^{\nu}(\vec{k}) \chi_{\mu \nu}(k)\right|_{k^{0}=|\vec{k}|}
$$

Using the above formula, the differential photon production rate can be rewritten as

$$
\frac{d \Gamma_{\gamma}}{d^{3} k}=\left.\frac{e^{2}}{(2 \pi)^{3} 2|\vec{k}|} \Phi(k) \sum_{s=1,2} \epsilon_{(s)}^{\mu}(\vec{k}) \epsilon_{(s)}^{\nu}(\vec{k}) \chi_{\mu \nu}(k)\right|_{k^{0}=|\vec{k}|},
$$


where $\epsilon_{(1)}^{\mu}$ and $\epsilon_{(2)}^{\mu}$ are mutually orthogonal polarization vectors that are also orthogonal to $k^{\mu}$. By the $S O(2)$ symmetry in the $x y$-plane of our model we can choose $\vec{k}$ to lie in the $x z$-plane - see Figure 6.7. Following [45,48], we set

$$
\vec{k}=k_{0}(\sin \vartheta, 0, \cos \vartheta) .
$$

With this choice of $\vec{k}$ the polarization vectors can be chosen as

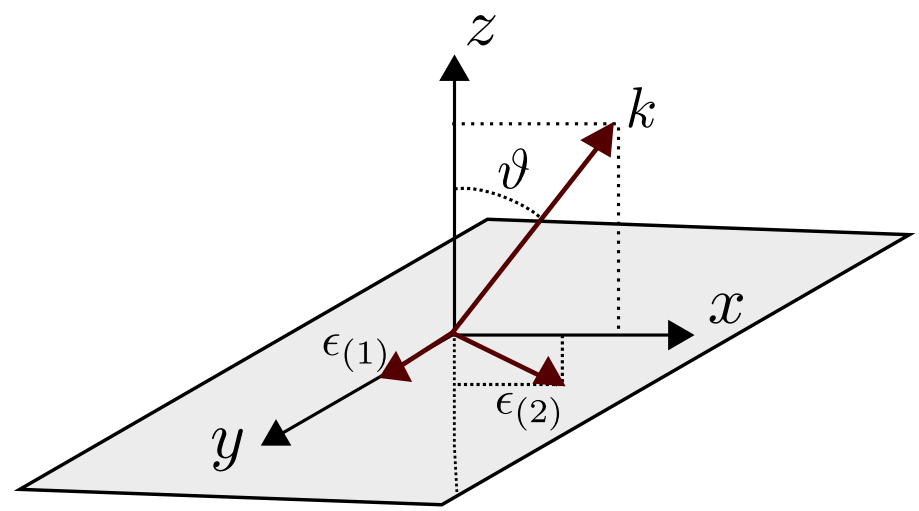

Figure 6.7: Momentum $\vec{k}$ and polarization vectors $\vec{\epsilon}_{(1)}$ and $\vec{\epsilon}_{(2)}$. The $S O(2)$ rotational symmetry in the $x y$-plane allows us to choose the momentum lying in the $x z$-plane, forming an angle $\vartheta$ with the $z$-direction. Both polarization vectors are orthogonal to $\vec{k}$. We chose $\vec{\epsilon}_{(1)}$ oriented along the $y$-direction and $\vec{\epsilon}_{(2)}$ contained in the xz-plane.

$$
\vec{\epsilon}_{(1)}=(0,1,0), \quad \vec{\epsilon}_{(2)}=(\cos \vartheta, 0,-\sin \vartheta) .
$$

For later purposes we split the trace of the spectral density into two parts

$$
\eta^{\mu \nu} \chi_{\mu \nu}=\chi_{(1)}+\chi_{(2)}
$$

where $\chi_{(s)}$ is proportional to the number of photons emitted with polarization $\vec{\epsilon}_{(s)}$. These quantities are given by

$$
\begin{aligned}
& \chi_{(1)}=\epsilon_{(1)}^{\mu} \epsilon_{(1)}^{\nu} \chi_{\mu \nu}=\chi_{y y} \\
& \chi_{(2)}=\epsilon_{(2)}^{\mu} \epsilon_{(2)}^{\nu} \chi_{\mu \nu}=\cos ^{2} \vartheta \chi_{x x}+\sin ^{2} \vartheta \chi_{z z}-2 \cos \vartheta \sin \vartheta \chi_{x z} .
\end{aligned}
$$

We now proceed to explain how to compute the retarded Green's function of two electromagnetic currents using holography. It turns out that the gravity theory dual to the field theory described by the Lagrangian $\mathcal{L}$ is simply obtained by adding a $U(1)$ kinetic term to the action (5.1). As we are dealing with a bottom-up model, we consider a five-dimensional 
$U(1)$ kinetic term of the form,

$$
S_{U(1)}=-K \int d^{5} x F_{m n} F^{m n}
$$

where $F_{m n}=\partial_{m} A_{n}-\partial_{n} A_{m}$ is the field strength associated to the gauge field $A_{m}$ ( $m=$ $0,1,2,3,4)$ and $K$ is a constant. ${ }^{11}$ Let $A_{\mu}(\mu=0,1,2,3)$ denote the components of this gauge field along the gauge theory coordinates $(t, \vec{x})$ and $A_{4}=A_{u}$ denote the component along the radial coordinate of $A d S$. In order to simplify our calculations, we gauge fix $A_{u}=0$. Our final results, however, will be written only in terms of gauge invariant quantities, in such a way that this gauge choice will not be relevant.

Given the translation invariance of our model, we can Fourier decompose the gauge field $A_{\mu}$ as

$$
A_{\mu}(t, \vec{x}, u)=\int \frac{d^{4} k}{(2 \pi)^{4}} e^{-i k^{0} t+i \vec{k} \cdot \vec{x}} A_{\mu}\left(k^{0}, \vec{k}, u\right) .
$$

The equations of motion derived from (6.46) are given by

$$
\partial_{\mu}\left(\sqrt{-g} g^{\mu \alpha} g^{\nu \beta} F_{\alpha \beta}\right)=0
$$

In terms of the gauge invariant quantities $E_{i}=\partial_{0} A_{i}-\partial_{y} A_{i}$, the above equations of motion split into a decoupled equation for $E_{y}$,

$$
E_{y}^{\prime \prime}+\left(\log \sqrt{-g} g^{u u} g^{y y}\right)^{\prime} E_{y}^{\prime}-\frac{\bar{k}^{2}}{g^{u u}} E_{y}=0,
$$

and a system of two coupled equations for $E_{x}$ and $E_{z},{ }^{12}$

$$
\begin{aligned}
& E_{x}^{\prime \prime}+\left[\left(\log \sqrt{-g} g^{u u} g^{x x}\right)^{\prime}-\left(\log \frac{g^{x x}}{g^{t t}}\right)^{\prime} \frac{k_{x}^{2}}{\bar{k}^{2}} g^{x x}\right] E_{x}^{\prime}-\frac{\bar{k}^{2}}{g^{u u}} E_{x}-\left(\log \frac{g^{x x}}{g^{t t}}\right)^{\prime} \frac{k_{z} k_{x}}{\bar{k}^{2}} g^{z z} E_{z}^{\prime}=0 \\
& E_{z}^{\prime \prime}+\left[\left(\log \sqrt{-g} g^{u u} g^{z z}\right)^{\prime}-\left(\log \frac{g^{z z}}{g^{t t}}\right)^{\prime} \frac{k_{z}^{2}}{\bar{k}^{2}} g^{z z}\right] E_{z}^{\prime}-\frac{\bar{k}^{2}}{g^{u u}} E_{z}-\left(\log \frac{g^{z z}}{g^{t t}}\right)^{\prime} \frac{k_{z} k_{x}}{\bar{k}^{2}} g^{x x} E_{x}^{\prime}=0
\end{aligned}
$$

where the primes denote derivatives with respect to $u$ and $\bar{k}^{2} \equiv g^{\alpha \beta} k_{\alpha} k_{\beta}$. Note that the above equations are written in momentum space.

\footnotetext{
${ }^{11}$ In top-down calculations, $K$ is proportional to the number of electrically charged degrees of freedom times the number of colors in the dual gauge theory. For instance, when photons are produced from adjoint matter we have $K \propto N_{c}^{2}$ [161], while for fundamental fields, $K \propto N_{c} N_{f}$ [45, 46, 48, 162]. In bottom-up models, this constant can be chosen freely and, since we are only interested in ratios of spectral densities (that are proportional to $K$ ), this constant will play no role in our analysis.

${ }^{12}$ In the derivation of the equations of motion for $E_{x}$ and $E_{z}$ we used the constraint $g^{\alpha \beta} k_{\alpha} A_{\beta}^{\prime}=0$.
} 
The action (6.46) can be written in terms of the gauge invariant fields $E_{i}$ as

$$
\begin{aligned}
S_{\epsilon}=-2 K \int d t d \vec{x} \frac{\sqrt{-g} g^{u u}}{k_{0}^{2} \bar{k}^{2}} & {\left[\left(-g^{t t} k_{0}^{2}-g^{z z} k_{z}^{2}\right) g^{x x} E_{x} E_{x}^{\prime}-\bar{k}^{2} g^{y y} E_{y} E_{y}^{\prime}+\right.} \\
+ & \left.g^{x x} g^{z z} k_{x} k_{z}\left(E_{x} E_{z}\right)^{\prime}+\left(-g^{t t} k_{0}^{2}-g^{x x} k_{x}^{2}\right) g^{z z} E_{z} E_{z}^{\prime}\right]_{u=\epsilon} .
\end{aligned}
$$

The retarded correlators are obtained by taking functional derivatives of the above action with respect to the boundary values of the gauge fields $A^{\mu(0)}$. In the computation of $\chi_{(1)}$ and $\chi_{(2)}$ we only need the spatial correlators $G_{x x}^{\mathrm{R}}, G_{y y}^{\mathrm{R}}, G_{z z}^{\mathrm{R}}$ and $G_{x z}^{\mathrm{R}}=G_{z x}^{\mathrm{R}}$. This correlators can be obtained in terms of the $E_{i}$ 's as

$$
G_{i j}^{\mathrm{R}}=\frac{\delta^{2} S_{\epsilon}}{\delta A^{i(0)} \delta A^{j(0)}}=k_{0}^{2} \frac{\delta^{2} S_{\epsilon}}{\delta E_{i}^{(0)} \delta E_{j}^{(0)}},
$$

where $E_{i}^{(0)}$ is the boundary value of the gauge field $E_{i}$.

As the mode $E_{y}$ does not couple to the other modes, the spectral density for photons with polarization $\vec{\epsilon}_{(1)}$ can be obtained by applying the prescription of [75]. The retarded correlator reads

$$
G_{y y}^{\mathrm{R}}=k_{0}^{2} \frac{\delta^{2} S_{\epsilon}}{\delta E_{y}^{(0) 2}}=-\left.\frac{4 K}{k_{0}^{2}} \sqrt{-g} g^{u u} g^{y y} \frac{E_{y}^{\prime}(k, u)}{E_{y}(k, u)}\right|_{u \rightarrow 0} .
$$

The corresponding spectral density is then given by

$$
\chi_{(1)}=\chi_{y y}=-2 \operatorname{Im} G_{y y}^{\mathrm{R}}=\frac{8 K}{k_{0}^{2}} \operatorname{Im}\left[\sqrt{-g} g^{u u} g^{y y} \frac{E_{y}^{\prime}(k, u)}{E_{y}(k, u)}\right]_{u \rightarrow 0} .
$$

The computation of $\chi_{(2)}$ is more involved, because of the coupling between $E_{x}$ and $E_{z}$. We face this problem by following the technique developed in [45] to deal with mixed operators. First, we write a near-boundary expression for the fields $E_{x}$ and $E_{z}$,

$$
\begin{aligned}
& E_{x}=E_{x}^{(0)}+u^{2} E_{x}^{(2)} \cos \vartheta+u^{4} E_{x}^{(4)}+O\left(u^{6}\right), \\
& E_{z}=E_{z}^{(0)}-u^{2} E_{x}^{(2)} \sin \vartheta+u^{4} E_{z}^{(4)}+O\left(u^{6}\right) .
\end{aligned}
$$

The form of the second order coefficients was chosen such that the equations of motion (6.50) are satisfied. The equations of motion also determine the coefficients $E_{x}^{(4)}$ and $E_{z}^{(4)}$ 
in terms of the lower order coefficients,

$$
\begin{aligned}
& E_{x}^{(4)}=\frac{a^{2} \lambda_{\mathrm{GB}} \cos \vartheta}{96\left(1-B_{0}\right)\left(1-4 \lambda_{\mathrm{GB}}\right)}\left(3 k_{0}^{2}\left(B_{0}-2 \lambda_{\mathrm{GB}}\right) E_{x}^{(0)} \cos \vartheta+8\left(1-2 B_{0}\right) E_{x}^{(2)}\right), \\
& E_{z}^{(4)}=\frac{a^{2}}{192 \sqrt{1-4 \lambda_{\mathrm{GB}}}}\left[3 k_{0}^{2}\left(\lambda_{\mathrm{GB}}-B_{0}\right)\left(E_{x}^{(0)} \sin \vartheta-E_{z}^{(0)} \cos \vartheta\right) \cos \vartheta-8 B_{0} E_{x}^{(2)} \sin \vartheta\right] .
\end{aligned}
$$

The remaining coefficients $E_{x}^{(0)}, E_{z}^{(0)}$ and $E_{x}^{(2)}$ can be extracted from the numerical solution. With the above expressions the boundary action (6.51) takes the form

$$
S_{\epsilon}=\sqrt{B_{0}} K\left[-\frac{1}{2}\left(E_{x}^{(0)} \sin \vartheta+E_{z}^{(0)} \cos \vartheta\right)^{2}-\frac{4}{B_{0} k_{0}^{2}}\left(E_{x}^{(0)} E_{x}^{(2)} \cos \vartheta+E_{z}^{(0)} E_{x}^{(2)} \sin \vartheta\right)\right]
$$

Finally, using (6.38), (6.45) and (6.52) we can show that

$$
\chi_{(2)}=\frac{16 K}{\sqrt{B_{0}}} \operatorname{Im}\left[\frac{\delta E_{x}^{(2)}}{\delta E_{x}^{(0)}} \cos \vartheta-\frac{\delta E_{x}^{(2)}}{\delta E_{z}^{(0)}} \sin \vartheta\right]
$$

where the functional derivatives $\delta E_{x}^{(2)} / \delta E_{x}^{(0)}$ and $\delta E_{x}^{(2)} / \delta E_{z}^{(0)}$ are calculated according to the prescription given in [45].

The trace of the spectral density $\chi_{\mu}^{\mu}=\chi_{(1)}+\chi_{(2)}$ is a function of the parameters $\left(\lambda_{\mathrm{GB}}, a, \vartheta, u_{\mathrm{H}}, k^{0}\right)$. In order to study the effects of the anisotropy parameter and the GaussBonnet coupling, we computed $\chi_{\mu}^{\mu}$ for several values of $\left(\lambda_{\mathrm{GB}}, a, \vartheta\right)$, choosing as normalization the isotropic result

$$
\chi_{\mathrm{iso}}=\chi_{\mu}^{\mu}\left(\lambda_{\mathrm{GB}}=0, a=0\right) .
$$

Our comparison with the isotropic result was made at fixed temperature $T_{0}=0.32 .{ }^{13}$ The results for the ratio $\chi_{\mu}^{\mu} / \chi_{\text {iso }}$ as a function of the dimensionless frequency $\mathrm{w}=k^{0} / 2 \pi T_{0}$ are presented in Figure 6.8. For an anisotropic plasma, we have $\chi_{(1)} \neq \chi_{(2)}$. However, in our case the smallness of the anisotropy parameter $a$ makes these two quantities almost equal, presenting a very similar behavior as a function of $\mathrm{w}$, so we chose to plot only the total spectral density instead of plotting the two spectral densities separately. At least, we observed that $\chi_{(1)}$ is slightly bigger than $\chi_{(2)}$, as was the case in $[45,48]$. We also verified that our results reproduce the calculations of [171] in the limit $a \rightarrow 0$ and that they are consistent with anisotropic calculations of [45] in the limit $\lambda_{\mathrm{GB}} \rightarrow 0$ and small values of

\footnotetext{
${ }^{13}$ Doing this, one must note that the temperature $T$ of the system is a function of $\left(\lambda_{\mathrm{GB}}, a, u_{\mathrm{H}}\right)$ and, consequently, it changes as we vary these parameters. Therefore, we need to ajust $u_{\mathrm{H}}$ in such a way that all the spectral densities are calculated at same temperature $T_{0}$, defined by $T_{0}=T\left(\lambda_{\mathrm{GB}}=0, a=0, u_{\mathrm{H}}=1\right)$.
} 
$a / T$

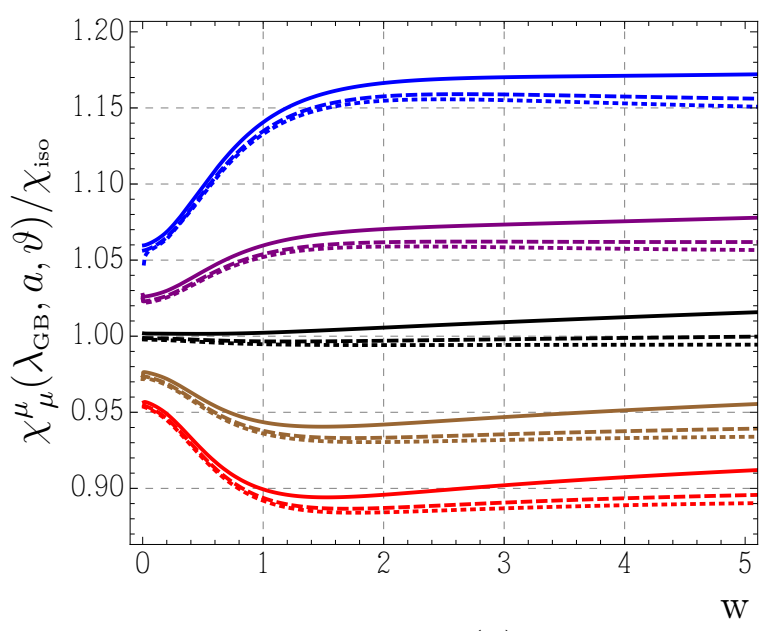

(a)

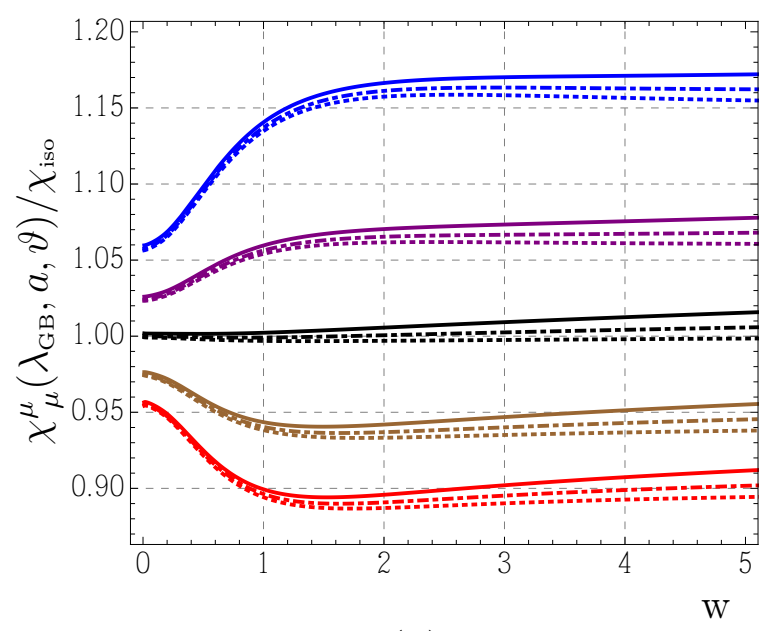

(b)

Figure 6.8: The trace of the spectral density $\chi_{\mu}^{\mu}\left(\lambda_{G B}, a, \vartheta\right)$ normalized with respect to the isotropic result (6.59). All the spectral densities were calculated at the same temperature $T_{0}=$ 0.316698. The color of the curves identify the value of the $\lambda_{G B}$ parameter as: red curves $\left(\lambda_{G B}=-0.1\right)$, brown curves $\left(\lambda_{G B}=-0.05\right)$, black curves $\left(\lambda_{G B}=0\right)$, purple curves $\left(\lambda_{G B}=0.05\right)$ and blue curves $\left(\lambda_{G B}=0.1\right)$. In $(a)$, the angle of emission is fixed $(\vartheta=0)$ and we have solid curves $(a=0.2)$, dashed curves $(a=0.1)$ and dotted curves $(a=0)$. In (b), the anisotropy is fixed $(a=0.2)$ and we have solid curves $(\vartheta=0)$, dot-dashed curves $(\vartheta=\pi / 4)$ and dotted curves $(\vartheta=\pi / 2)$.

From Figure 6.8 it is clear that the effect of the Gauss-Bonnet coupling is to increase or decrease the photon production rate, depending on whether $\lambda_{\mathrm{GB}}>0$ or $\lambda_{\mathrm{GB}}<0$, respectively. The main effect of the anisotropy parameter is to increase the photon production rate. At small frequencies, $\chi_{\mu}^{\mu}$ does not depend strongly on $a$. For generic frequencies, the $\chi_{\mu}^{\mu}$ is higher for photons with longitudinal wave vectors $(\vartheta=0)$ than for the ones with transverse wave vectors $(\vartheta=\pi / 2)$. One qualitative difference between the corrections introduced by $\lambda_{\mathrm{GB}}$ and $a$ is their dependence on the frequency. Looking at the curves for $a=0$ in Figure 6.8, we see that the Gauss-Bonnet correction reaches a constant value after a sufficiently large value of $\mathrm{w}$. On the other hand, the effect of the anisotropy parameter $a$ is enhanced as we increase $\mathrm{w}$.

It is also interesting to analyze how the anisotropy and the Gauss-Bonnet term affects the total photon production (6.37), which can be expressed as

$$
\frac{-1}{4 K e^{2} T_{0}^{3}} \frac{d \Gamma_{\gamma}}{d \cos \vartheta d k^{0}}=\frac{\mathrm{w}}{32 K \pi^{3} T_{0}^{2}} \frac{1}{e^{2 \pi \mathrm{w}}-1}\left(\chi_{(1)}+\chi_{(2)}\right)
$$

This quantity is shown in Figure 6.9, for different values of $\lambda_{\mathrm{GB}}$ and $\vartheta$. From Figure 6.9 we see that, for $\lambda_{\mathrm{GB}}>0$, the peak in the spectrum of photons becomes higher, widens 
and gets shifted to the right. For $\lambda_{\mathrm{GB}}<0$, the peak becomes smaller, sharpens and gets shifted to the left. This should be contrasted with the results of [169] for a top-down higher derivative correction of the form $\alpha^{\prime 3} R^{4}$, where the peak in the spectrum becomes higher, sharpens and gets shifted to the left, approaching the weak coupling result [161], which shows a very sharp peak at small $\mathrm{w}$ in the photon spectrum. Therefore, the inclusion of the $\alpha^{\prime 3} R^{4}$ correction (which corresponds to a finite 't Hooft coupling correction in the gauge theory) goes into the direction of the weak coupling results, while this does not seems to be possible in the case of Gauss-Bonnet. However, a partial agreement between these two types of corrections is found when $\lambda_{\mathrm{GB}}<0$, where the peak in the photon spectrum sharpens and moves to the left, but it also becomes smaller, contrary to what happens at weak coupling. We can understand this partial agreement noting that, for $\lambda_{\mathrm{GB}}<0$, the ratio $\eta / s=\left(1-4 \lambda_{\mathrm{GB}}\right) /(4 \pi)$ increases, which also happens with $\eta / s$ when finite 't Hooft coupling corrections were taking into account. Since at weak coupling the shear viscosity over the entropy density ratio is proportional to the mean free path of momentum isotropization, we can associate the approaching of the weak coupling results (negative $\lambda_{\mathrm{GB}}$ corrections or $\alpha^{\prime}$-corrections) with a larger mean free path in both cases.

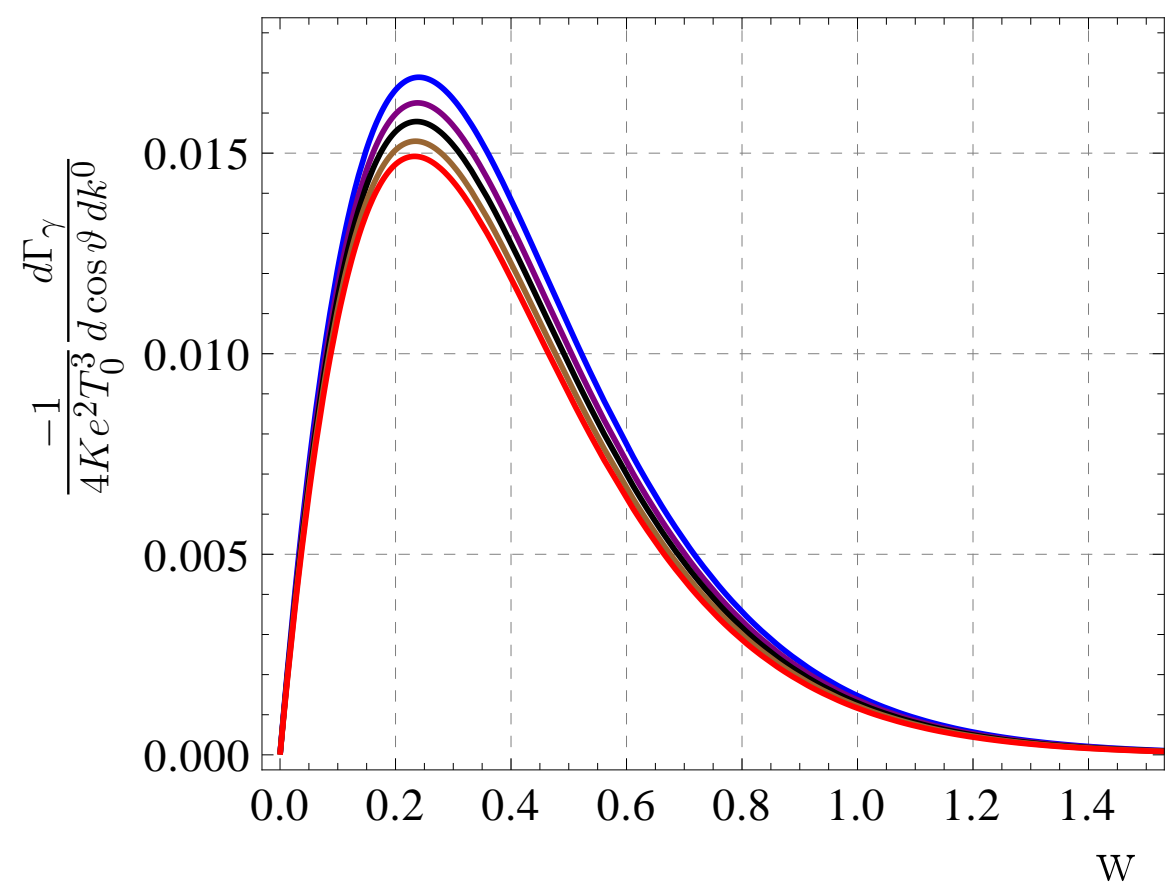

Figure 6.9: Total photon production rate as function of $w=k^{0} / 2 \pi T_{0}$. From top to bottom, the value of the Gauss-Bonnet coupling is identified as $\lambda_{G B}=0.1$ (blue), $\lambda_{G B}=0.05$ (purple), $\lambda_{G B}=0$ (black), $\lambda_{G B}=-0.05$ (brown), $\lambda_{G B}=-0.1$ (red). We have fixed $\vartheta=0$ and $a=0.2$. The results for different angles are very similar to the plot above due to the smallness of the anisotropy. 


\subsection{Discussion}

We have computed the shear viscosity over entropy density ratio for the dual plasma and found that the KSS bound [76] is violated, as expected from previous works where either the case $\left(a=0, \lambda_{\mathrm{GB}} \neq 0\right)[97]$ or the case $\left(a \neq 0, \lambda_{\mathrm{GB}}=0\right)$ [29] were considered. We also have studied how the anisotropy and higher curvature terms affect several other observables relevant to the study of the QGP, namely, the drag force, the jet quenching parameter, the quarkonium static potential and the photon production rate. In the gravity side, the anisotropy was introduced by an external source (an axion linear in the beam direction) that keeps the system in an equilibrium anisotropic state, while the higher curvature correction was chosen to be the Gauss-Bonnet term.

The effect of the Gauss-Bonnet term in our results are consistent with previous results $[159,171,174,175]$ and they are summarized in Table 6.1 , where we specify if the value of the observable increases or decreases compared to the case of isotropic $\mathcal{N}=4$ SYM. In this table we also present the result for the shear viscosity over entropy density obtained previously [55] and the finite 't Hooft corrections of type $\alpha^{\prime 3} R^{4}$ for these observables $[114,169,176,177]$.

Table 6.1: Summary of the effect of the Gauss-Bonnet coupling $\lambda_{G B}$ on several observables. We also present the finite 't Hooft corrections of type $\alpha^{\prime 3} R^{4}$. The comparison is taken w.r.t. the respective $\mathcal{N}=4 S Y M$ result at same temperature.

\begin{tabular}{|c|c|c|c|c|c|}
\hline & $\eta / s$ & Drag force & Jet quenching & Screening length & Photon prod. \\
\hline \hline$\lambda_{\mathrm{GB}}>0$ & decrease & increase & increase & decrease & increase \\
$\lambda_{\mathrm{GB}}<0$ & increase & decrease & decrease & increase & decrease \\
$\alpha^{\prime 3} R^{4}$ & increase & increase & decrease & decrease & increase \\
\hline
\end{tabular}

A possible heuristic interpretation of the increasing/decreasing in the above observables is to correlate these results with the changes in the ratio $\eta / s$. At weak coupling, $\eta / s$ is proportional to the mean free path of momentum isotropization of the plasma $\left(\eta / s \sim \ell_{\mathrm{mfp}}\right)$. Imagining a situation where the mean free path is decreasing, we should expect an external probe to interact more with the medium, increasing the energy loss of the probe and its probability to suffer scattering. As a result, we would obtain an increase in the drag force and the jet quenching parameter. Moreover, a low mean free path would break the connection between a quark-antiquark pair more easily, resulting in a low value of screening length. Finally, a low mean free path would raise the number of collisions per time and, consequently, the number of photons produced in these interactions would increase. Note that this situation matches exactly the case of $\lambda_{\mathrm{GB}}>0$. Of course, the opposite idea applies for $\lambda_{\mathrm{GB}}<0$. Although this reasoning seems to be consistent for the 
Gauss-Bonnet, it does not work when applied to the $\alpha^{\prime 3} R^{4}$ correction.

The effect of the anisotropy is similar to what was found previously $[31,32,37,38,45,51]$. The photon production rate and the quarkonium dissociation length in an anisotropic plasma are bigger than the corresponding quantities in an isotropic plasma at the same temperature. The drag force and the jet quenching parameter in an anisotropic plasma can be bigger or smaller than its isotropic counterparts, depending on several parameters like the quark velocity, the direction of the quark motion, and the direction of momentum broadening. Below we also summarize the effects of the anisotropy with a comparison between the value of the observables along the anisotropic direction (II) and along the transverse plane $(\perp)$ :

- Shear viscosity: $\quad \eta_{\perp}>\eta_{\|}$,

- Drag force: $\quad F_{\text {drag }}^{\perp}<F_{\text {drag }}^{\|}$,

- Jet quenching parameter: $\hat{q}_{\perp}<\hat{q}_{\|}$,

- Screening length: $L_{\perp}>L_{\|}$,

- Photon production rate: $\chi_{\mu \perp}^{\mu}<\chi_{\mu \|}^{\mu}$

The same interpretation in terms of the mean free path for the Gauss-Bonnet term can be applied here. Considering the mean free path in the anisotropic direction $\ell_{\mathrm{mfp}}^{\|}$and in the transverse plane $\ell_{\mathrm{mfp}}^{\perp}$, we note that the mean free path of an anisotropic system in the transverse plane is larger than the corresponding quantity in the anisotropic direction, because $\eta_{\perp}>\eta_{\|}$. This can be associated with a smaller drag force, a smaller jet quenching parameter, less screening (larger screening length), a smaller drag force and less photon production in the transverse plane when compared with the corresponding quantities in the anisotropic direction. 


\section{Chapter 7}

\section{Conclusion}

We have studied Lovelock gravity within the context of the AdS/CFT correspondence. Lovelock gravity is interesting from a purely gravity point of view as a natural generalization of Einstein's general relativity to higher dimensions, but our main motivation here relies on the fact that higher curvature terms arise as stringy corrections to supergravity and they are related, via the AdS/CFT correspondence, to finite 't Hooft coupling corrections in the dual gauge theory. Lovelock gravity can then be used as a simple model to study the effect of higher curvature terms in the dual field theory. Although we still do not know whether Lovelock gravity can be obtained from a string theory compactification, our phylosophy here is to work in this simpler scenario to gain insight about the effects of these higher curvature terms and perhaps uncover some universal properties. As reviewed in Chapter 3, Lovelock gravity has brought us significant results so far, such as the first counter-example to the famous KSS bound of the shear viscosity over entropy density. Moreover, it has revealed interesting connections between positivity of energy and causality constraints, and also showed that the avoidance of ghosts can be mapped into the requirement of unitarity in the dual CFT.

The first result of our work was the holographic computation of the so called ChernSimons diffusion coefficient, which is an important transport coefficient that parametrizes the rate of transition among degenerate vacua of a gauge theory. We worked in the gravity setup of Gauss-Bonnet gravity, and we also considered another class of higher curvature gravity, namely, Quasi-topological gravity. We found that the transport coefficient can increase or decrease the value obtained from pure Einstein's gravity, depending on the value of the couplings.

Next, in Chapter 5, we changed to a different gravity setup where the action is composed by an axion-dilation system with the addition of the Gauss-Bonnet term. The axion field was chosen to be linear in one of the spatial direction, corresponding to a dual gauge theory displaying anisotropy. The combination of the anisotropy with the higher curva- 
ture term significantly increased the difficult of the equations of motion, but we still were able to solve them analytically, for small anisotropies. One of our main concerns was to carry out the holographic renormalization, which is crucial to the extraction of correlation functions since the on-shell gravity action usually suffers from divergences. We employed the Hamilton-Jacobi approach of holographic renormalization. From a practical point of view, the main advantage of this method is that it is very general and the equations that appear are simply algebraic, allowing us to sistematically implement the procedure in softwares like Mathematica. Although the Hamilton-Jocobi approach showed itself to be very powerful, we found some technical subtleties related to the construction of the Hamiltonian. Firstly, the renormalization was possible only for small values of the Gauss-Bonnet coupling, and secondly we were not able to extract the Ward identities. This was due to the difficulty to write the Hamiltonian as a function of canonical momenta instead of the extrinsic curvature, requiring the inversion of nonlinear equations that unfortunately we were not able to accomplish.

Finally, in the last chapter we focused on the phenomenological application of the gravity solution that we obtained as the gravity dual of a strongly coupled anisotropic plasma. Motivated by the fact that the plasma produced in heavy ion collision experiments is anisotropic, we studied the effect of the Gauss-Bonnet term on several observables relevant to the study of the quark-gluon plasma. Our results were compatible to what we would expect from previous studies regarding only the anisotropy and only the Gauss-Bonnet term separately. Even though the results were not surprising, the main contribution of this work is perhaps to put the results for each observable all together and try to interpret them physically. Indeed, we presented a heuristic interpretation for the effect on the observables in terms of the shear viscosity. One of the most noticeble points of this analysis is that the results for Gauss-Bonnet gravity behave differently from those obtained from the known type IIB supergravity correction with schematic form $\alpha^{\prime 3} R^{4}$. Since higher curvature terms can have different effects on the observables, we can see how important is to understand these terms if we want a quantitative holographic description of real-world QGP, since soon or later we will have to deal with higher curvature corrections to have a plasma at non-infinite 't Hooft coupling.

There are still much more interesting aspects left to explore. Regarding the new anisotropic gravity solution we have found, we can still study how the anisotropy and the higher derivative terms affect other observables like the imaginary part of the quarkonium potential, the quarkonium dissociation length in a plasma wind, Langevin diffusion coefficients, the dilepton production rate or the elliptic flow of photons and dileptons, to name a few. Also, it would be interesting to see how these observables behave for similar models. As far as we are aware, the only model that incorporates both the anisotropy 
and the higher curvature correction is [121]. Besides the applications to strongly coupled gauge theories, which were one of the main concerns of this thesis, it would be nice to discover whether Lovelock gravity can be obtained from a string compactification, and of course determine the exact dual field theory. This is probably the most difficult task to accomplish, but centainly the most desirable one from a theoretical perspective. 


\section{Appendix A}

\section{Symmetries in quantum field theories}

\section{The Poincaré algebra}

Symmetries play a central hole in physics. In nature, the Poincaré symmetry expresses the homogeneity of spacetime and Lorentz invariance. The Poincaré group is the group of isometries of $\mathbb{R}^{3,1}$ and it includes ten generators: the four generators of translations $P_{\mu}$ and the six generators of the Lorentz transformations $M_{\mu \nu}$. The Poincare algebra has the form

$$
\begin{aligned}
{\left[P_{\mu}, P_{\nu}\right] } & =0 \\
{\left[M_{\mu \nu}, P_{\lambda}\right] } & =i\left(\eta_{\nu \lambda} P_{\mu}-\eta_{\mu \lambda} P_{\nu}\right) \\
{\left[M_{\mu \nu}, M_{\rho \sigma}\right] } & =i\left(\eta_{\nu \rho} M_{\mu \sigma}+\eta_{\mu \sigma} M_{\nu \rho}-\eta_{\mu \rho} M_{\nu \sigma}-\eta_{\nu \sigma} M_{\mu \rho}\right) .
\end{aligned}
$$

One immediate question would be if there is a more general symmetry behind the Poicaré symmetry, i.e., if the Poincaré algebra can be extended to a maximal symmetry group. For many years, that was believed to not be possible, due to the Coleman-Mandula theorem that we state below.

\section{Coleman-Mandula theorem}

The Coleman-Mandula theorem assumes that the S-matrix is based on a local relativistic quantum field theory in four-dimensional spacetime and that there is a mass gap between the vacuum and the one particle states. The conclusion of the theorem is that we cannot extend the Poicaré group including internal symmetries and maintain at the same time a non-trivial S-matrix. The proof of this theorem is obtained by considering the S-matrix of the theory and its transformation properties under Lie algebras. However, there is a loophole in this theorem: in the proof of the theorem it was implicitly assumed 
that the extension was bosonic, but we can consider fermionic generators as well. That was exactly what brought us to supersymmetry.

\section{Supersymmetry (SUSY)}

The loophole in the Coleman-Mandula theorem allows us to enlarge the Poincaré algebra by introducing supercharges

$$
\begin{aligned}
& Q_{\alpha}^{I}, \quad \alpha=1,2 \quad \text { Weyl spinor, } \\
& \bar{Q}_{\dot{\alpha} I}=\left(Q_{\alpha}^{I}\right)^{\dagger}, \quad \dot{\alpha}=1,2 \quad \text { anti-Weyl spinor }
\end{aligned}
$$

where $I=1, \ldots, \mathcal{N}$. The case of $\mathcal{N}=1$ is referred to as the minimal SUSY. In four dimensions, the maximal supersymmetry we can have in order to have only particles with spin lower or equal than one is $\mathcal{N}=4$. In total we have $4 \mathcal{N}$ supercharges. In addition to the Poincaré algebra, we have the commutation relations

$$
\begin{aligned}
{\left[P_{\mu}, Q_{\alpha}\right] } & =0, \quad\left[P_{\mu}, \bar{Q}^{\dot{\alpha}}\right]=0 \\
{\left[M_{\mu \nu}, Q_{\alpha}\right] } & =i\left(\sigma^{\mu \nu}\right)_{\alpha}^{\beta}, \quad\left[M_{\mu \nu}, \bar{Q}^{\dot{\alpha}}\right]=i\left(\bar{\sigma}^{\mu \nu}\right)_{\beta}^{\dot{\alpha}} \bar{Q}^{\dot{\beta}} .
\end{aligned}
$$

where $\sigma^{\mu \nu}=\frac{i}{2}\left[\gamma^{\mu}, \gamma^{\nu}\right]$ with $\gamma^{\prime}$ s being the usual gamma matrices satisfying the Clifford algebra $\left\{\gamma^{\mu}, \gamma^{\nu}\right\}=2 \eta^{\mu \nu}$. Spinorial generators, due to their fermionic nature, have anticommutation relations instead of commutation relations

$$
\left\{Q_{\alpha}, \bar{Q}_{\dot{\beta}}\right\}=2\left(\sigma^{\mu}\right)_{\alpha \dot{\beta}} P_{\mu}
$$

where $\sigma^{\mu}$ are the Pauli matrices.

\section{Conformal symmetry}

Another possibility to avoid the Coleman-Mandula is in the case where the theory has only massless states, and so the Poincaré algebra can be enlarged to the conformal algebra. A conformal transformation $x \mapsto f(x)$ is such that the metric transforms as

$$
g_{\mu \nu}(x) \mapsto \Omega^{2}(x) g_{\mu \nu}(x)
$$

In addition to the ten generators of the Poincaré algebra, the conformal algebra contains one dilatation $D$ and four special conformal transformations $K_{\mu}$, summing to a total of fifteen generators that represent the conformal group $S O(4,2)$. In addition to the 
commutation relations of the Poincaré algebra, we have

$$
\begin{aligned}
& {\left[M_{\mu \nu}, K_{\rho}\right]=i\left(\eta_{\mu \rho} K_{\nu}-\eta_{\nu \rho} K_{\mu}\right),} \\
& {\left[D, K_{\mu}\right]=-i K_{\mu}, \quad\left[D, P_{\mu}\right]=i P_{\mu}, \quad\left[D, M_{\mu \nu}\right]=0,} \\
& {\left[K_{\mu}, K_{\nu}\right]=0, \quad\left[K_{\mu}, P_{\nu}\right]=-2 i\left(\eta_{\mu \nu} D-M_{\mu \nu}\right) .}
\end{aligned}
$$

\section{Superconformal algebra}

If the supersymmetric theory is also conformal, the algebra can be extended to the superconformal algebra. In addition to the generators of the conformal group $M_{\mu \nu}, P_{\mu}, D$ and $K_{\mu}$ and to the supercharges $Q_{\alpha}^{I}, \bar{Q}_{\dot{\alpha}}^{I}$, it is necessary to include additional fermionic generators $S_{\alpha}^{I}, \bar{S}_{\dot{\alpha}}^{I}$ in order to close the algebra. We can think the $S$ fermionic generators as the supersymmetric partners of $K_{\mu}$, in the same way as the $Q$ generators are the supersymmetric partners of $P_{\mu}$.

In the case $\mathcal{N}=4$ which is particularly interesting from the point of view of the AdS/CFT correspondence, in this case we have sixteen $Q$ 's plus sixteen $S$ 's equal thirty two supercharges. Putting all together we obtain the $S U(2,2 \mid 4)$ superalgebra. 


\section{Appendix B}

\section{Derivation of the anisotropic gravity solution}

In this Appendix we give some details on how we have found our solution (5.8) and present its explicit expression.

The Einstein equations (5.3) are diagonal, as a consequence of the fact that the metric only depends on $u$. We have then four equations for the metric (since the $x x$ - and $y y$ components are not independent) plus the equation for the dilaton in (5.5). There are four fields to solve for: $\phi, F, B$, and $H$. Plugging the Ansatz (5.6)-(5.8) into the equations and expanding to order $O\left(a^{2}\right)$ one finds that the equation for $\phi_{2}(u)$ decouples. It reads

$$
\phi_{2}^{\prime \prime}+\frac{u F_{0}^{\prime}-3 F_{0}}{u F_{0}} \phi_{2}^{\prime}=\frac{1}{F_{0}}
$$

with $F_{0}$ given by (5.9). This can be readily solved changing coordinates as

$$
u \rightarrow U(u)=\sqrt{1-4 \lambda_{\mathrm{GB}}\left(1-\frac{u^{4}}{u_{\mathrm{H}}^{4}}\right)}
$$

in intermediate steps. The two integration constants are fixed in such a way that $\phi_{2}$ is regular at the horizon and vanishes at the boundary, $\phi_{2, \text { bdry }}=0$. One finds

$$
\begin{aligned}
& \phi_{2}(u)=-\frac{u_{\mathrm{H}}^{2}}{8}\left[\alpha+U(u)+\log \left(1+\frac{u^{2}}{u_{\mathrm{H}}^{2}}\right)^{2}\right. \\
& \left.-\sqrt{\lambda}_{\mathrm{GB}} \log \left(U(u)+2{\sqrt{\lambda_{\mathrm{GB}}}}_{\frac{u^{2}}{u_{\mathrm{H}}^{2}}}\right)^{2}-\log \left(U(u)+1-4 \lambda_{\mathrm{GB}}\left(1+\frac{u^{2}}{u_{\mathrm{H}}^{2}}\right)\right)\right] \text {, }
\end{aligned}
$$


where

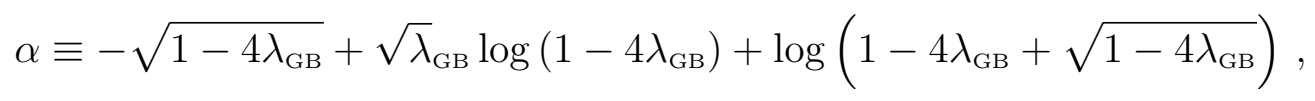

and $U(u)$ is defined as above. We notice that $U$ is always positive (since $\lambda_{\mathrm{GB}}<1 / 4$ ), and so is the argument of the last logarithm in (B.3). When $\lambda_{\mathrm{GB}}=0$ we recover the result of [23], see eq. (164) of that paper.

To find $H_{2}$, we take the difference of the $x x$ - and $z z$-components of (5.3). One obtains a decoupled equation that reads

$$
H_{2}^{\prime \prime}(u)+p(u) H_{2}^{\prime}(u)=q(u)
$$

with

$$
\begin{aligned}
& p(u)=\frac{3\left(1-4 \lambda_{\mathrm{GB}}\right)(U(u)-1)+4 \lambda_{\mathrm{GB}}(3 U(u)-5) u^{4} / u_{\mathrm{H}}^{4}}{u U(u)^{2}(1-U(u))}, \\
& q(u)=\frac{2 \lambda_{\mathrm{GB}} U(u)}{\left(1-4 \lambda_{\mathrm{GB}}\right)(1-U(u))} .
\end{aligned}
$$

This equation can be integrated readily via (B.2), fixing the integration constants as above. In particular we request that $H_{2, \text { bdry }}=0$. The final result is

$$
\begin{aligned}
& H_{2}(u)=\frac{u_{\mathrm{H}}^{2}}{8\left(1-4 \lambda_{\mathrm{GB}}\right)}\left[\beta+U(u)+\log \left(1+\frac{u^{2}}{u_{\mathrm{H}}^{2}}\right)+2 \lambda_{\mathrm{GB}} \frac{u^{2}}{u_{\mathrm{H}}^{2}}\left(\frac{u^{2}}{u_{\mathrm{H}}^{2}}-2\right)\right. \\
& \left.-\sqrt{\lambda}_{\mathrm{GB}} \log \left(U(u)+2 \sqrt{\lambda}_{\mathrm{GB}} \frac{u^{2}}{u_{\mathrm{H}}^{2}}\right)^{2}-\log \left(\frac{U(u)+1-4 \lambda_{\mathrm{GB}}\left(1+\frac{u^{2}}{u_{\mathrm{H}}^{2}}\right)}{U(u)-1+4 \lambda_{\mathrm{GB}}\left(1+\frac{u^{2}}{u_{\mathrm{H}}^{2}}\right)}\right)^{1 / 2}\right],
\end{aligned}
$$

where, again, we have left $U(u)$ implicit in some places for compactness and where

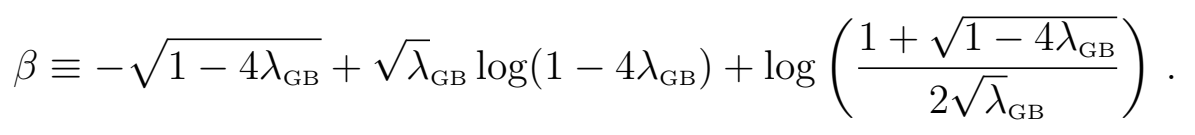

Similarly we can solve for the other fields. More specifically, now that we know $\phi_{2}$ and $H_{2}$, we can use the $t t$-component of (5.3) to obtain $F_{2}$ and the $u u$-component to obtain $B_{2}$. One can finally check that the $x x$ - and $z z$-components are also solved separately, as expected because of the Bianchi identities. The explicit expressions for the equations are not particularly illuminating, so that we limit ourselves to reporting the final results for 
the remaining fields, which are given by

$$
\begin{aligned}
& F_{2}(u)=\frac{u_{\mathrm{H}}^{2}}{12\left(1-4 \lambda_{\mathrm{GB}}\right) U(u)}\left(\frac{u}{u_{\mathrm{H}}}\right)^{4}\left[\gamma+U(u)+\left(1-4 \lambda_{\mathrm{GB}}\right)\left(\frac{u_{\mathrm{H}}}{u}\right)^{2}\right. \\
& +4 \lambda_{\mathrm{GB}}\left(\frac{u}{u_{\mathrm{H}}}\right)^{2}-6 \lambda_{\mathrm{GB}}\left(\frac{u}{u_{\mathrm{H}}}\right)^{4}+\log \left(1+\frac{u^{2}}{u_{\mathrm{H}}^{2}}\right)^{2} \\
& \left.-\sqrt{\lambda}_{\mathrm{GB}} \log \left(U(u)+2{\sqrt{\lambda_{\mathrm{GB}}}}_{\frac{u^{2}}{u_{\mathrm{H}}^{2}}}\right)^{2}-\log \left(U(u)+1-4 \lambda_{\mathrm{GB}}\left(1+\frac{u^{2}}{u_{\mathrm{H}}^{2}}\right)\right)\right] \text {, }
\end{aligned}
$$

with

$$
\gamma \equiv-2+6 \lambda_{\mathrm{GB}}+\sqrt{\lambda}_{\mathrm{GB}} \log \left(1+2{\sqrt{\lambda_{\mathrm{GB}}}}^{2}+\log \left(\frac{1-4 \lambda_{\mathrm{GB}}}{2}\right)\right.
$$

and by

$$
\begin{aligned}
& B_{2}(u)=\frac{u_{\mathrm{H}}^{2}}{24\left(1-4 \lambda_{\mathrm{GB}}\right)}\left[\alpha+U(u) \frac{u_{\mathrm{H}}^{2}-u^{2}}{u_{\mathrm{H}}^{2}+u^{2}}+\log \left(1+\frac{u^{2}}{u_{\mathrm{H}}^{2}}\right)^{2}\right. \\
& -\frac{2 u^{2}}{u_{\mathrm{H}}^{2}+u^{2}}\left(1-2 \lambda_{\mathrm{GB}}+\lambda_{\mathrm{GB}}\left(\frac{u}{u_{\mathrm{H}}}\right)^{2}+3 \lambda_{\mathrm{GB}}\left(\frac{u}{u_{\mathrm{H}}}\right)^{4}\right) \\
& \left.-\sqrt{\lambda}_{\mathrm{GB}} \log \left(U(u)+2{\sqrt{\lambda_{\mathrm{GB}}}}_{\frac{u^{2}}{u_{\mathrm{H}}^{2}}}\right)^{2}-\log \left(U(u)+1-4 \lambda_{\mathrm{GB}}\left(1+\frac{u^{2}}{u_{\mathrm{H}}^{2}}\right)\right)\right] \text {. }
\end{aligned}
$$

Again, we have fixed the integration constants in such a way that the fields be regular at the horizon and vanish at the boundary, $F_{2 \text {,bdry }}=B_{2 \text {,bdry }}=0$. Notice also that $F_{2}\left(u_{\mathrm{H}}\right)=0$, as it should be for a blackening factor. One can check that when $\lambda_{\mathrm{GB}}=0$ the results from [22] are recovered. ${ }^{1}$

\footnotetext{
${ }^{1}$ In order to do so, one needs to take into account the different Ansätze and include a factor of the dilaton in (5.6), according to eq. (8) of [23].
} 


\section{Appendix C}

\section{Shear viscosity from Kubo formula}

In this Appendix we report a alternative derivation of the shear viscosity tensor (6.19). As is well known (see e.g. [75, 178-180]), the shear viscosity can be also computed using a Kubo formula

$$
\eta=\lim _{\omega \rightarrow 0} \frac{1}{\omega} \operatorname{Im} G_{\mathrm{R}}(\omega, \vec{k}=0),
$$

where $G_{\mathrm{R}}(k)$ is the retarded Green's function for the stress tensor. First, we take metric fluctuations $h_{m n}$ around our solution and linearize the equations of motion. Here, we are interested in the modes $\psi_{\perp}=h_{y}^{x}$ and $\psi_{\|}=h_{z}^{x}$. In momentum space, we have

$$
\psi(u, x)=\int \frac{d^{4} k}{(2 \pi)^{4}} J(k) \psi(u ; k) e^{-i k_{i} x^{i}}, \quad k_{i}=(-\omega, \mathbf{k})
$$

where $\psi$ denotes generically one of the modes $\psi_{\perp}$ or $\psi_{\|}$. The prescription tells us to solve the equation for $\psi(u ; k)$ imposing infalling boundary conditions and regularity at the horizon and satisfying $\psi=1$ at the boundary.

To compute the shear viscosity, we can restrict ourselves to zero spatial momentum and small frequency $\omega$. For simplicity, we also consider small $\lambda_{\mathrm{GB}}$. The linearized equations for $\psi(u ; \omega)$ have the form

$$
K_{0}(u) \psi^{\prime \prime}+K_{0}^{\prime}(u) \psi^{\prime}=0,
$$

where for $\psi=\psi_{\perp}$ we have, up to orders $O\left(a^{4}, \lambda_{\mathrm{GB}}^{2}, \omega^{2}\right)$,

$$
\begin{aligned}
& K_{0}^{\perp}(u)= \frac{u^{4}\left(a^{2} u_{\mathrm{H}}^{2} \log 2+6\right)-a^{2} u_{\mathrm{H}}^{6} \log \left(1+\frac{u^{2}}{u_{\mathrm{H}}^{2}}\right)-6 u_{\mathrm{H}}^{4}}{12 u^{3} u_{\mathrm{H}}^{4}} \\
&+\frac{\lambda_{\mathrm{GB}}}{12 u^{3} u_{\mathrm{H}}^{8}}\left[u^{8}\left(a^{2} u_{\mathrm{H}}^{2}(5-6 \log 2)-18\right)-u^{4} u_{\mathrm{H}}^{4}\left(a^{2} u_{\mathrm{H}}^{2}(2-5 \log 2)-6\right)\right. \\
&\left.\quad-4 a^{2} u^{6} u_{\mathrm{H}}^{4}+\left(12+a^{2} u^{2}\right) u_{\mathrm{H}}^{8}+a^{2} u_{\mathrm{H}}^{2}\left(3 u^{8}-2 u_{\mathrm{H}}^{8}\right) \log \left(1+\frac{u^{2}}{u_{\mathrm{H}}^{2}}\right)\right],
\end{aligned}
$$


and for $\psi=\psi_{\|}$we have

$$
\begin{aligned}
K_{0}^{\|}(u)= & K_{0}^{\perp}(u)+\frac{a^{2}\left(u_{\mathrm{H}}^{4}-u^{4}\right) \log \left(1+\frac{u^{2}}{u_{\mathrm{H}}^{2}}\right)}{8 u^{3} u_{\mathrm{H}}^{2}} \\
& +\frac{a^{2} \lambda_{\mathrm{GB}}\left(-7 u^{8}+10 u^{6} u_{\mathrm{H}}^{2}-u^{4} u_{\mathrm{H}}^{4}-2 u^{2} u_{\mathrm{H}}^{6}+2\left(3 u^{8}-5 u^{4} u_{\mathrm{H}}^{4}+2 u_{\mathrm{H}}^{8}\right) \log \left(1+\frac{u^{2}}{u_{\mathrm{H}}^{2}}\right)\right)}{16 u^{3} u_{\mathrm{H}}^{6}} .
\end{aligned}
$$

The equations above can be solved by considering an Ansatz of the form

$$
\psi(u ; \omega)=\left(1-\frac{u^{4}}{u_{\mathrm{H}}^{4}}\right)^{-\frac{i \omega}{4 \pi T}}\left[1+\omega\left(f_{0}(u)+\lambda_{\mathrm{GB}}\left(f_{1}(u)+a^{2} f_{2}(u)\right)\right)+O\left(a^{4}, \lambda_{\mathrm{GB}}^{2}, \omega^{2}\right)\right]
$$

where $T$ is the temperature given by (5.13). The functions $f_{0}(u), f_{1}(u)$ and $f_{2}(u)$ can be determined by substituting the Ansatz into the linearized equation and solving order by order. The resulting expressions are not particularly illuminating and we do not report them here. The next step is to compute the quadratic on-shell action, which turns out to be a surface term of the form

$$
S_{\text {on-shell }}^{(2)}=-\left.\frac{1}{2} \int \frac{d^{4} k}{(2 \pi)^{4}} J(k) \mathcal{F}(u ; k) J(-k)\right|_{u \rightarrow 0} ^{u=u_{\mathrm{H}}},
$$

with $\mathcal{F}(u, k)=\frac{1}{16 \pi G} K_{0}(u) \psi^{\prime}(u ; k) \psi(u ;-k)$. The prescription of [75] instructs us to take only the contribution of the boundary. The retarded Green's function is then given by

$$
G_{\mathrm{R}}(k)=\lim _{u \rightarrow 0} \mathcal{F}(u ; k)
$$

Finally, using (C.1) and the result for the entropy (5.14) we can compute the ratio of the shear viscosity over entropy density

$$
\begin{aligned}
& \frac{\eta_{\perp}}{s}=\frac{1-4 \lambda_{\mathrm{GB}}}{4 \pi}+a^{2} \lambda_{\mathrm{GB}} \frac{u_{\mathrm{H}}^{2}}{8 \pi}+O\left(a^{4}, \lambda_{\mathrm{GB}}^{2}\right), \\
& \frac{\eta_{\|}}{s}=\frac{1-4 \lambda_{\mathrm{GB}}}{4 \pi}+a^{2}\left(3 \lambda_{\mathrm{GB}}-2 \log 2\right) \frac{u_{\mathrm{H}}^{2}}{32 \pi}+O\left(a^{4}, \lambda_{\mathrm{GB}}^{2}\right) .
\end{aligned}
$$

These results agree with the ones obtained via the membrane paradigm expanded to first order in $\lambda_{\mathrm{GB}}$. 


\section{Appendix D}

\section{Drag force for a general background and arbitrary direction}

In this appendix we derive a formula for the drag force. The metric background is assumed to be of the form

$$
d s^{2}=G_{t t} d t^{2}+G_{x x}\left(d x^{2}+d y^{2}\right)+G_{z z} d z^{2}+G_{u u} d u^{2}
$$

We will only assume rotational symmetry in the $x y$ directions and consider the metric to depend only on $u$. This is essentially what was done in [32], but here we consider the motion of the quark along an arbitrary direction, as in [31].

The Nambu-Goto action for the string is given by

$$
S=-\frac{1}{2 \pi \alpha^{\prime}} \int d \tau d \sigma e^{\phi / 2} \sqrt{-\operatorname{det} g}=\int d \tau d \sigma \mathcal{L}
$$

where $\phi=\phi(u)$ is the dilaton field. By rotational symmetry in the $x y$ directions we can set $y=0$. We choose static gauge $(t, u)=(\tau, \sigma)$ and let us consider the motion of the quark in the $x z$ plane with a string embedding

$$
x(t, u)=(v t+\xi(u)) \sin \varphi, \quad z(t, u)=(v t+\zeta(u)) \cos \varphi
$$

where $\varphi$ is the angle of the quark trajectory with the $z$ axis, i.e., $\varphi=0$ corresponds to the motion parallel with the anisotropic direction and $\varphi=\pi / 2$ corresponds to the motion in the transversal direction. The boundary conditions are $\xi(u \rightarrow 0)=0$ and $\zeta(u \rightarrow 0)=0$, which are necessary in order to reproduce the stationary motion of the quark.

First, we need to compute the induced metric $g_{\alpha \beta}=G_{\mu \nu} \partial_{\alpha} x^{\mu} \partial_{\beta} x^{\nu}$ on the string 
worldsheet,

$$
g_{\alpha \beta}=\left(\begin{array}{cc}
G_{t t}+v^{2}\left(G_{z z} \cos ^{2} \varphi+G_{x x} \sin ^{2} \varphi\right) & v\left(G_{z z} \zeta^{\prime}(u) \cos ^{2} \varphi+G_{x x} \xi^{\prime} \sin ^{2} \varphi\right) \\
v\left(G_{z z} \zeta^{\prime} \cos ^{2} \varphi+G_{x x} \xi^{\prime} \sin ^{2} \varphi\right) & G_{u u}+G_{z z} \zeta^{\prime 2} \cos ^{2} \varphi+G_{x x} \xi^{\prime 2} \sin ^{2} \varphi
\end{array}\right)
$$

where the prime denotes derivative w.r.t. $u$. Ignoring factors of $\frac{1}{2 \pi \alpha^{\prime}}$, the Lagrangian takes the form

$$
\begin{aligned}
\mathcal{L}=-e^{\phi / 2}\left[-G_{z z} \cos ^{2} \varphi\left(\zeta^{\prime 2} G_{t t}+G_{u u} v^{2}+G_{x x} v^{2}\left(\zeta^{\prime}-\xi^{\prime}\right)^{2} \sin ^{2} \varphi\right)-\right. & \\
& \left.-G_{x x} \sin ^{2} \varphi\left(G_{t t} \xi^{\prime 2}+G_{u u} v^{2}\right)-G_{t t} G_{u u}\right]^{\frac{1}{2}} .
\end{aligned}
$$

And we have associated (conserved) momentum flow

$$
\begin{aligned}
\Pi_{x} & =\frac{\delta \mathcal{L}}{\delta x^{\prime}}=-\frac{e^{\phi} G_{x x} \sin \varphi}{\mathcal{L}}\left(G_{t t} \xi^{\prime}-G_{z z} v^{2}\left(\zeta^{\prime}-\xi^{\prime}\right) \cos ^{2} \varphi\right) \\
\Pi_{z} & =\frac{\delta \mathcal{L}}{\delta z^{\prime}}=-\frac{e^{\phi} G_{z z} \cos \varphi}{\mathcal{L}}\left(G_{t t} \zeta^{\prime}+G_{x x} v^{2}\left(\zeta^{\prime}-\xi^{\prime}\right) \sin ^{2} \varphi\right)
\end{aligned}
$$

The values of the momenta will be fixed by imposing that $\xi^{\prime}$ and $\zeta^{\prime}$ are both real. To do this, we invert the above expression to write

$$
\xi^{\prime}(u)=\frac{G_{z z} N_{x}}{G_{x x} N_{z}} \zeta^{\prime}(u)
$$

where we have defined the quantities

$$
\begin{aligned}
& N_{x}=G_{t t} \Pi_{x} \csc (\varphi)+G_{x x} v^{2}\left(\Pi_{x} \sin (\varphi)+\Pi_{z} \cos (\varphi)\right), \\
& N_{z}=G_{t t} \Pi_{z} \sec (\varphi)+G_{z z} v^{2}\left(\Pi_{x} \sin (\varphi)+\Pi_{z} \cos (\varphi)\right)
\end{aligned}
$$

Then we can use, for example, the expression for $\Pi_{z}$ to obtain $\zeta^{\prime}$. The final expressions we found are given by

$$
\xi^{\prime}= \pm \sqrt{-\frac{2 G_{u u} G_{z z}}{G_{t t} G_{x x} D}} N_{x}, \quad \zeta^{\prime}= \pm \sqrt{-\frac{2 G_{u u} G_{x x}}{G_{t t} G_{z z} D}} N_{z}
$$

where

$$
\begin{array}{r}
D=2 G_{t t}\left(G_{x x} \Pi_{z}^{2}+G_{z z} \Pi_{x}^{2}\right)+G_{x x} G_{z z}\left[G_{t t} e^{\phi}\left(2 G_{t t}+v^{2}\left(G_{x x}+G_{z z}\right)\right)+v^{2}\left(\Pi_{x}^{2}+\Pi_{z}^{2}\right)\right]+ \\
+G_{x x} G_{z z} v^{2}\left[\left(-G_{t t}\left(G_{x x}-G_{z z}\right) e^{\phi}-\Pi_{x}^{2}+\Pi_{z}^{2}\right) \cos (2 \varphi)+2 \Pi_{x} \Pi_{z} \sin (2 \varphi)\right] . \quad \text { (D.12) }
\end{array}
$$

There is a sign ambiguity here, which we will fix later. The condition that $\xi^{\prime}$ and $\zeta^{\prime}$ are always real can be satisfied only if $D$ is positive for all $u$. But, in general, $D$ has two 
zeros (turning points). Thus, in order to satisfy the positivity condition the two zeros should coincide at some critical point $u_{c}$. Also, the numerators $N_{x}$ and $N_{z}$ should vanish at the same point $u_{c}$, because otherwise $\xi^{\prime}$ and $\zeta^{\prime}$ would diverge. We begin the analysis finding the zeros of the numerator. Imposing $N_{x}$ and $N_{z}$ to vanish at $u_{c}$ gives us a relation between $\Pi_{x}$ and $\Pi_{z}$,

$$
\frac{\Pi_{x}}{\Pi_{z}}=\left.\frac{G_{x x}}{G_{z z}} \tan \varphi\right|_{u=u_{c}} .
$$

Using this relation, we can find the two zeros of $D$ at $u_{c}$. This gives us two equations, the first one is

$$
\left[\frac{2 G_{t t}}{v^{2}}+G_{x x}+G_{z z}+\left(G_{z z}-G_{x x}\right) \cos (2 \varphi)\right]_{u=u_{c}}=0
$$

which can be used to fix the value of the critical point $u_{c}$. The second equation completely fixes the values of $\Pi_{x}$ and $\Pi_{z}$ and gives us the drag force

$$
\Pi_{x}=\left.e^{\phi / 2} G_{x x} v \sin \varphi\right|_{u=u_{c}}, \quad \Pi_{z}=\left.e^{\phi / 2} G_{z z} v \cos \varphi\right|_{u=u_{c}} .
$$

We have fixed the sign of the momenta to be positive, thus $\xi^{\prime}$ and $\zeta^{\prime}$ are both negative, which is consistent with the physical condition that the string "trails" behind the quark $[122,123]$ and not in front of it. Two special cases are obtained from (6.22) by setting $\varphi=0$ and $\varphi=\pi / 2$. This gives us the drag force parallel and transversal to the direction of motion of the quark, respectively

$$
F_{\text {drag }}^{\|}=\left.e^{\phi / 2} G_{z z} v\right|_{u=u_{c}}, \quad F_{\text {drag }}^{\perp}=\left.e^{\phi / 2} G_{x x} v\right|_{u=u_{c}} .
$$




\section{Appendix E}

\section{Jet quenching parameter for an arbitrary motion}

In this appendix we derive a formula for $\hat{q}$ considering a motion in an arbitrary direction and generic background. The steps are basically the same of [37], but here the computation is carried out in Einstein frame and the metric is left generic, with only a few assumptions that we will specify below.

We assume a five-dimensional background displaying rotational symmetry in the $x y$ directions,

$$
d s^{2}=G_{t t} d t^{2}+G_{x x}\left(d x^{2}+d y^{2}\right)+G_{z z} d z^{2}+G_{u u} d u^{2} .
$$

From the rotational symmetry we can choose the direction of motion within the $x z$ plane. We define rotated coordinates

$$
\begin{aligned}
& z=Z \cos \theta-X \sin \theta, \\
& x=Z \sin \theta+X \cos \theta, \\
& y=Y .
\end{aligned}
$$

The new coordinates $(X, Y, Z)$ are obtained from the old coordinates $(x, y, z)$ by a rotation of an angle $\theta$ around the $y$-axis. We choose $Z$ to be the direction of motion of the quark. The direction of the momentum broadening takes place in the $X Y$ plane and it forms an angle $\varphi$ with the $Y$-axis. The prescription instructs us to consider a string with an endpoint moving at the speed of light along the $Z$ direction. The other endpoint is separated by a small distance $\ell$ along the direction of the momentum broadening. Thus we have a string worldsheet whose boundary is a rectangular light-like Wilson loop with sizes $L^{-}$(along the $Z^{-}$direction) and $\ell$, where $L^{-}$is assumed to be very large. Our task is to find a string worldsheet that minimizes the Nambu-Goto action satisfying this boundary condition. We then need to evaluate the on-shell Nambu-Goto action and expand it to second order 
in $\ell$ to obtain

$$
\left\langle W^{A}(\mathcal{C})\right\rangle \simeq \exp \left[-\frac{L^{-} \ell^{2}}{4 \sqrt{2}} \hat{q}\right]
$$

from where we extract the jet quenching parameter. It is convenient to work in light-cone coordinates

$$
t=\frac{Z^{-}+Z^{+}}{\sqrt{2}}, \quad Z=\frac{Z^{-}-Z^{+}}{\sqrt{2}} .
$$

The metric in these new coordinates takes the form

$$
G_{\mu \nu}^{(L C)}=\left(\begin{array}{rrrrr}
G_{++} & G_{+-} & G_{X-} & 0 & 0 \\
G_{+-} & G_{++} & -G_{X-} & 0 & 0 \\
G_{X-} & -G_{X-} & G_{X X} & 0 & 0 \\
0 & 0 & 0 & G_{x x} & 0 \\
0 & 0 & 0 & 0 & G_{u u}
\end{array}\right)
$$

where

$$
\begin{aligned}
G_{++} & =\frac{1}{2}\left(G_{t t}+G_{x x} \sin ^{2} \theta+G_{z z} \cos ^{2} \theta\right), \\
G_{+-} & =\frac{1}{2}\left(G_{t t}-G_{x x} \sin ^{2} \theta-G_{z z} \cos ^{2} \theta\right), \\
G_{X-} & =\frac{\sin \theta \cos \theta}{\sqrt{2}}\left(G_{x x}-G_{z z}\right), \\
G_{X X} & =G_{x x} \cos ^{2} \theta+G_{z z} \sin ^{2} \theta
\end{aligned}
$$

We choose the static gauge $(\tau, \sigma)=\left(Z^{-}, u\right)$. Since we are assuming $L^{-}$to be very large, there is a translational symmetry in the $Z^{-}$direction, and we can fix the string embedding to only depend on $u$,

$$
Z^{+}=Z^{+}(u), \quad X \rightarrow X(u) \sin \varphi, \quad Y \rightarrow Y(u) \cos \varphi
$$

The induced metric on the string worldsheet $g_{\alpha \beta}=G_{\mu \nu} \partial_{\alpha} x^{\mu} \partial_{\beta} x^{\nu}$ is given by

$$
\begin{aligned}
& g_{\tau \tau}=G_{++}, \quad g_{\tau \sigma}=\sin \varphi G_{X-} X^{\prime}+G_{+-}\left(Z^{+}\right)^{\prime} \\
& g_{\sigma \sigma}=G_{u u}+\sin ^{2} \varphi G_{X X} X^{\prime 2}-2 \sin \varphi G_{X-}\left(Z^{+}\right)^{\prime} X^{\prime}+\cos ^{2} \varphi G_{x x} Y^{\prime 2}+G_{++}\left(Z^{+}\right)^{\prime 2}
\end{aligned}
$$

where the primes denote derivative w.r.t. $u$. We can now write the Nambu-Goto action, ${ }^{1}$

$$
S=-2 \frac{L^{-}}{2 \pi \alpha^{\prime}} \int_{0}^{u_{\mathrm{H}}} d u e^{\phi / 2} \sqrt{-\operatorname{det} g} \equiv \frac{L^{-}}{\pi \alpha^{\prime}} \int_{0}^{u_{\mathrm{H}}} d u \mathcal{L}
$$

\footnotetext{
${ }^{1}$ The extra factor of 2 comes from the two branches of the string worldsheet.
} 
where $\phi=\phi(u)$ is the dilaton scalar field and

$$
\begin{aligned}
\mathcal{L}=-e^{\phi / 2} & {\left[\left(G_{+-}+G_{++}\right)\left(2 G_{X-} X^{\prime}\left(Z^{+}\right)^{\prime} \sin \varphi-G_{X X}\left(Z^{+}\right)^{\prime 2}\right)\right.} \\
& \left.-G_{++}\left(G_{u u}+G_{x x} Y^{\prime 2} \cos ^{2} \varphi\right)+X^{\prime 2} \sin ^{2} \varphi\left(G_{X-}^{2}-G_{++} G_{X X}\right)\right]^{\frac{1}{2}} .
\end{aligned}
$$

Since the Lagrangian does not depend on $Z^{+}, X$ and $Y$, we have three conserved quantities, given by the canonical conjugate momenta. Up to a constant factor, they are given by

$$
\begin{aligned}
\Pi_{+} & =\frac{e^{\phi}}{\mathcal{L}}\left(G_{+-}+G_{++}\right)\left(G_{X-} X^{\prime} \sin \varphi-G_{X X}\left(Z^{+}\right)^{\prime}\right) \\
\Pi_{X} & =\frac{e^{\phi}}{\mathcal{L}} \sin \varphi\left[G_{X-}\left(Z^{+}\right)^{\prime}\left(G_{+-}+G_{++}\right)+X^{\prime} \sin \varphi\left(G_{X-}^{2}-G_{++} G_{X X}\right)\right], \\
\Pi_{Y} & =-\frac{e^{\phi}}{\mathcal{L}} G_{++} G_{x x} Y^{\prime} \cos ^{2} \varphi
\end{aligned}
$$

We are interested in the limit where $\Pi_{+}, \Pi_{X}$ and $\Pi_{Y}$ are small. ${ }^{2}$ Working in first order in the $\Pi_{+}, \Pi_{X}$ and $\Pi_{Y}$, we can invert the above expressions to obtain $\left(Z^{+}\right)^{\prime}, X^{\prime}$ and $Y^{\prime}$, we find that

$$
\begin{aligned}
\left(Z^{+}\right)^{\prime} & =c_{++} \Pi_{+}+c_{+X} \Pi_{X} \csc \varphi, \\
X^{\prime} & =c_{X+} \Pi_{+} \csc \varphi+c_{X X} \Pi_{X} \csc ^{2} \varphi \\
Y^{\prime} & =c_{Y Y} \Pi_{Y} \sec ^{2} \varphi
\end{aligned}
$$

where

$$
\begin{aligned}
& c_{++}=\frac{e^{-\phi / 2} G_{u u}\left(G_{X-}^{2}-G_{++} G_{X X}\right)}{\left(G_{+-}+G_{++}\right) \sqrt{-G_{++} G_{u u}}\left(G_{X X}^{2}-2 G_{X-}^{2}\right)}, \\
& c_{+X}=c_{X+}=\frac{e^{-\phi / 2} G_{u u} G_{X-}}{\sqrt{-G_{++} G_{u u}}\left(2 G_{X-}^{2}-G_{X X}^{2}\right)}, \\
& c_{X X}=-\frac{e^{-\phi / 2} G_{u u} G_{X X}}{\sqrt{-G_{++} G_{u u}}\left(G_{X X}^{2}-2 G_{X-}^{2}\right)}, \\
& c_{Y Y}=-\frac{e^{-\phi / 2} G_{u u}}{G_{x x} \sqrt{-G_{++} G_{u u}}} .
\end{aligned}
$$

Integration of $Z^{+^{\prime}}$ gives zero. Integration of $X^{\prime}$ gives $\ell / 2$. Integration of $Y^{\prime}$ also gives $\ell / 2$.

\footnotetext{
${ }^{2}$ This is a consequence of $\ell$ be small, as explained in [37].
} 
The conclusion is that

$$
\begin{aligned}
\Pi_{+} & =\frac{\ell \sin \varphi\left(\int_{0}^{u_{\mathrm{H}}} c_{+X}(u) d u\right)}{2\left(\left(\int_{0}^{u_{\mathrm{H}}} c_{+X}(u) d u\right)^{2}-\left(\int_{0}^{u_{\mathrm{H}}} c_{++}(u) d u\right) \int_{0}^{u_{\mathrm{H}}} c_{+X}(u) d u\right)}, \\
\Pi_{X} & =\frac{\ell \sin ^{2} \varphi\left(\int_{0}^{u_{\mathrm{H}}} c_{++}(u) d u\right)}{2\left(\int_{0}^{u_{\mathrm{H}}} c_{++}(u) d u\right) \int_{0}^{u_{\mathrm{H}}} c_{+X}(u) d u-2\left(\int_{0}^{u_{\mathrm{H}}} c_{+X}(u) d u\right)^{2}}, \\
\Pi_{Y} & =\frac{\ell \cos ^{2} \varphi}{2 \int_{0}^{u_{\mathrm{H}}} c_{Y Y}(u) d u} .
\end{aligned}
$$

The on-shell action then takes the form, up to second order in the momenta,

$$
S=2 i \frac{\sqrt{\lambda} L^{-}}{4 \pi} \int_{0}^{u_{\mathrm{H}}} d u\left[c_{++} \Pi_{+}^{2}+\frac{1}{\sin ^{2} \varphi} c_{X X} \Pi_{X}^{2}+\frac{2}{\sin \varphi} c_{+X} \Pi_{+} \Pi_{X}+\frac{1}{\cos ^{2} \varphi} c_{Y Y} \Pi_{Y}^{2}\right] .
$$

Using the expressions for the coefficients the action can be rewritten as

$$
S=2 i \frac{\sqrt{\lambda} L^{-} \ell^{2}}{16 \pi}\left(\hat{P}(\theta) \sin ^{2} \varphi+\hat{Q}(\theta) \cos ^{2} \varphi\right)
$$

where

$$
\begin{aligned}
\hat{P}(\theta) & \equiv \frac{\int_{0}^{u_{\mathrm{H}}} c_{++}(u) d u}{\left(\int_{0}^{u_{\mathrm{H}}} c_{++}(u) d u\right) \int_{0}^{u_{\mathrm{H}}} c_{+X}(u) d u-\left(\int_{0}^{u_{\mathrm{H}}} c_{+X}(u) d u\right)^{2}}, \\
\hat{Q}(\theta) & \equiv \frac{1}{\int_{0}^{u_{\mathrm{H}}} c_{Y Y} d u} .
\end{aligned}
$$

From this we immediately extract the jet quenching parameter

$$
\hat{q}=\frac{\sqrt{2 \lambda}}{\pi}\left(\hat{P}(\theta) \sin ^{2} \varphi+\hat{Q}(\theta) \cos ^{2} \varphi\right) .
$$




\section{Appendix F}

\section{Quarkonium static potential in generic background}

In this appendix we derive a formula for the static potential of a quark-antiquark pair (quarkonium). ${ }^{1}$ Let $L$ be the separation between the quarks and assume a generic background of the form (D.1). The dual picture corresponds to an U-shaped open string whose endpoints are located at the boundary and are identified with the quarks. Our task is to find the string worldsheet that minimizes the Nambu-Goto action (D.2). By rotational symmetry in the $x y$-plane we can assume the pair to live in the $x z$ plane. Putting the center of mass of the pair at the origin, let $q$ be the polar radial coordinate and $\theta$ the angle between the pair and the $z$ direction. We fix the gauge $(\tau, \sigma)=(t, q)$. In this way the string embedding has the form

$$
X^{\mu}=(\tau, \sigma \sin \theta, 0, \sigma \cos \theta, u(\sigma))
$$

The induced metric on the string worldsheet is given by

$$
g_{\tau \tau}=G_{t t}, \quad g_{\sigma \sigma}=G_{p p}+G_{u u} u^{\prime 2}, \quad g_{\tau \sigma}=0
$$

where $G_{p p} \equiv G_{z z} \cos ^{2} \theta+G_{x x} \sin ^{2} \theta$ and the prime denotes derivative w.r.t. $\sigma$. The NambuGoto action takes the form

$$
S=-\frac{\mathcal{T}}{2 \pi \alpha^{\prime}} \int_{-L / 2}^{L / 2} d \sigma e^{\phi / 2} \sqrt{-G_{t t}\left(G_{p p}+G_{u u} u^{\prime 2}\right)} \equiv \int_{-L / 2}^{L / 2} d \sigma \mathcal{L} .
$$

\footnotetext{
${ }^{1}$ This computation is similar to what was done in [32], generalizing the prescription of [181] for an anisotropic background.
} 
Since the Lagrangean $\mathcal{L}$ does not depend explicitly on $\sigma$, the Hamiltonian is a constant of motion

$$
H=\frac{\partial \mathcal{L}}{\partial \sigma^{\prime}} \sigma^{\prime}-\mathcal{L}=-\frac{\mathcal{T}}{2 \pi \alpha^{\prime}} \frac{e^{\phi / 2} G_{t t} G_{p p}}{\sqrt{-G_{t t}\left(G_{p p}+G_{u u} u^{\prime 2}\right)}} .
$$

Evaluating the Hamiltonian at the turning point $u_{0} \equiv u(0)$, where $u^{\prime}=0$, we find the value of the constant to be

$$
C=\left.\frac{\mathcal{T}}{2 \pi \alpha^{\prime}} e^{\frac{\phi}{2}} \sqrt{-G_{p p} G_{t t}}\right|_{u=u_{0}}
$$

In order to simplify the expressions, it is useful to define the auxiliary quantities

$$
P \equiv e^{\frac{\phi}{2}} \sqrt{-G_{p p} G_{t t}}, \quad Q \equiv e^{\frac{\phi}{2}} \sqrt{-G_{t t} G_{u u}}
$$

Using (F.4) and(F.5) we can find an expression for $u^{\prime},{ }^{2}$

$$
u^{\prime}= \pm \frac{P}{Q} \frac{\sqrt{P^{2}-P_{0}^{2}}}{P_{0}},\left.\quad P_{0} \equiv P\right|_{u=u_{0}}
$$

Integrating the above expression we find that the separation between the quarks is given by

$$
L=-2 \int_{0}^{u_{0}} \frac{d \sigma}{d u} d u=2 \int_{0}^{u_{0}} \frac{Q}{P} \frac{P_{0}}{\sqrt{P^{2}-P_{0}^{2}}} .
$$

Before we compute the on-shell Nambu-Goto action to find the potential energy that keeps the pair bounded, we need to take care of the ultraviolet divergence due to the infinite mass of the quarks. The mass term corresponds to a string hanging down straight from the boundary to the horizon. Note that in this case the string goes from 0 to $u_{\mathrm{H}}$ while $\sigma$ is fixed, thus it effectively corresponds to $u^{\prime} \rightarrow \infty$. Expanding the on-shell Nambu-Goto action in powers of $1 / u^{\prime}$ for this configuration we obtain

$$
M_{Q}=-\frac{\mathcal{T}}{2 \pi \alpha^{\prime}} \int_{0}^{u_{\mathrm{H}}} d u Q+O\left(\frac{1}{u^{\prime}}\right)
$$

Finally, computing the on-shell Nambu-Goto action for the U-shaped configuration with the mass subtraction we obtain the static potential

$$
V_{Q \bar{Q}}=\frac{S^{(\text {on-shell })}}{\mathcal{T}}-2 M_{Q}=-\frac{1}{2 \pi \alpha^{\prime}}\left[P_{0} L+2 \int_{0}^{u_{0}} d u \frac{Q}{P}\left(\sqrt{P^{2}-P_{0}^{2}}-P\right)-2 \int_{u_{0}}^{u_{\mathrm{H}}} d u Q\right]
$$

\footnotetext{
${ }^{2}$ One needs to be careful with the choice of sign here: when $\sigma$ goes from 0 to $L / 2$, then $u$ goes from $u_{0}$ to 0 and thus $u^{\prime}<0$.
} 


\section{Bibliography}

[1] J. M. Maldacena, "The large $N$ limit of superconformal field theories and supergravity," Adv. Theor. Math. Phys. 2, 231 (1998) [Int. J. Theor. Phys. 38, 1113 (1999)] [hep-th/9711200]. 1

[2] S. S. Gubser, I. R. Klebanov, A. M. Polyakov, "Gauge theory correlators from noncritical string theory," Phys. Lett. B428, 105-114 (1998) [hep-th/9802109]. 1

[3] E. Witten, "Anti-de Sitter space and holography," Adv. Theor. Math. Phys. 2, 253291 (1998) [hep-th/9802150]. 1

[4] J. Adams et al. [STAR Collaboration], "Experimental and theoretical challenges in the search for the quark gluon plasma: The STAR Collaboration's critical assessment of the evidence from RHIC collisions," Nucl. Phys. A 757, 102 (2005) [nucl-ex/0501009]. 1

[5] K. Adcox et al. [PHENIX Collaboration], "Formation of dense partonic matter in relativistic nucleus-nucleus collisions at RHIC: Experimental evaluation by the PHENIX collaboration," Nucl. Phys. A 757, 184 (2005) [nucl-ex/0410003]. 1

[6] Proceedings of Quark Matter 2011: J. Phys. GG 38, number 12 (December 2011). 1

[7] S. S. Gubser, I. R. Klebanov and A. A. Tseytlin, "Coupling constant dependence in the thermodynamics of $\mathrm{N}=4$ supersymmetric Yang-Mills theory," Nucl. Phys. B 534, 202 (1998) doi:10.1016/S0550-3213(98)00514-8 [hep-th/9805156]. 1

[8] D. Lovelock, "The Einstein tensor and its generalizations," J. Math. Phys. 12, 498 (1971). 1, 21, 35, 36

[9] B. Zwiebach, "Curvature Squared Terms and String Theories," Phys. Lett. B 156, 315 (1985). 1, 23, 35

[10] D. G. Boulware and S. Deser, "String Generated Gravity Models," Phys. Rev. Lett. 55, 2656 (1985). 1, 23, 27, 29, 35

[11] R. -G. Cai, "Gauss-Bonnet black holes in AdS spaces," Phys. Rev. D 65, 084014 (2002) [hep-th/0109133]. 1, 35

[12] T. Padmanabhan and D. Kothawala, "Lanczos-Lovelock models of gravity," Phys. Rept. 531, 115 (2013) [arXiv:1302.2151 [gr-qc]]. 1 
[13] J. D. Edelstein, "Lovelock theory, black holes and holography," Springer Proc. Math. Stat. 60, 19 (2014) [arXiv:1303.6213 [gr-qc]]. 1, 25, 27, 35, 43, 60

[14] X. O. Camanho, J. D. Edelstein and J. M. Sanchez De Santos, "Lovelock theory and the AdS/CFT correspondence," Gen. Rel. Grav. 46, 1637 (2014) [arXiv:1309.6483 [hep-th]]. 1, 27, 35

[15] X. O. Camanho and J. D. Edelstein, "Causality in AdS/CFT and Lovelock theory," JHEP 1006, 099 (2010) [arXiv:0912.1944 [hep-th]]. 2, 30, 32, 37

[16] X. O. Camanho, J. D. Edelstein, G. Giribet and A. Gomberoff, "Generalized phase transitions in Lovelock gravity," Phys. Rev. D 90, 064028 (2014) [arXiv:1311.6768 [hep-th]]. 2, 3, 40

[17] X. O. Camanho and J. D. Edelstein, "A Lovelock black hole bestiary," Class. Quant. Grav. 30, 035009 (2013) [arXiv:1103.3669 [hep-th]]. 2, 27, 35, 37

[18] M. J. Duff, "Observations on Conformal Anomalies," Nucl. Phys. B 125, 334 (1977). 2,3

[19] S. 'i. Nojiri and S. D. Odintsov, "On the conformal anomaly from higher derivative gravity in AdS / CFT correspondence," Int. J. Mod. Phys. A 15, 413 (2000) [hepth/9903033]. 2, 3, 40

[20] M. Blau, K. S. Narain and E. Gava, "On subleading contributions to the AdS / CFT trace anomaly," JHEP 9909, 018 (1999) [hep-th/9904179]. 2, 3, 40

[21] T. Azeyanagi, W. Li and T. Takayanagi, "On String Theory Duals of Lifshitz-like Fixed Points," JHEP 0906, 084 (2009) [arXiv:0905.0688 [hep-th]]. 2, 43, 45

[22] D. Mateos and D. Trancanelli, "The anisotropic N=4 super Yang-Mills plasma and its instabilities," Phys. Rev. Lett. 107, 101601 (2011) [arXiv:1105.3472 [hep-th]]. 2, $3,40,41,42,43,44,45,59,60,61,63,65,92$

[23] D. Mateos and D. Trancanelli, "Thermodynamics and Instabilities of a Strongly Coupled Anisotropic Plasma," JHEP 1107, 054 (2011) [arXiv:1106.1637 [hep-th]]. 2, 3, $42,43,44,45,59,60,61,91,92$

[24] I. Papadimitriou, "Holographic Renormalization of general dilaton-axion gravity," JHEP 1108, 119 (2011) [arXiv:1106.4826 [hep-th]]. 2, 20, 45, 46, 48, 49, 50, 52, 56, 59

[25] J. T. Liu and W. A. Sabra, "Hamilton-Jacobi Counterterms for Einstein-GaussBonnet Gravity," Class. Quant. Grav. 27, 175014 (2010) [arXiv:0807.1256 [hep-th]]. $2,46,47,48,56$

[26] E. Shuryak, "Why does the quark gluon plasma at RHIC behave as a nearly ideal fluid?," Prog. Part. Nucl. Phys. 53, 273 (2004) [arXiv:hep-ph/0312227]. 2

[27] E. V. Shuryak, "What RHIC experiments and theory tell us about properties of quark-gluon plasma?," Nucl. Phys. A 750, 64 (2005) [arXiv:hep-ph/0405066]. 2 
[28] J. Casalderrey-Solana, H. Liu, D. Mateos, K. Rajagopal and U. A. Wiedemann, "Gauge/String Duality, Hot QCD and Heavy Ion Collisions," arXiv:1101.0618 [hepth]. 3

[29] A. Rebhan and D. Steineder, "Violation of the Holographic Viscosity Bound in a Strongly Coupled Anisotropic Plasma," Phys. Rev. Lett. 108, 021601 (2012) [arXiv:1110.6825 [hep-th]]. 3, 40, 63, 65, 82

[30] K. A. Mamo, "Holographic Wilsonian RG Flow of the Shear Viscosity to Entropy Ratio in Strongly Coupled Anisotropic Plasma," JHEP 1210, 070 (2012) [arXiv:1205.1797 [hep-th]]. 3, 63

[31] M. Chernicoff, D. Fernandez, D. Mateos and D. Trancanelli, "Drag force in a strongly coupled anisotropic plasma," JHEP 1208, 100 (2012) [arXiv:1202.3696 [hep-th]]. 3, $66,67,68,83,95$

[32] D. Giataganas, "Probing strongly coupled anisotropic plasma," JHEP 1207, 031 (2012) [arXiv:1202.4436 [hep-th]]. 3, 66, 70, 71, 73, 83, 95, 102

[33] S. Chakraborty and N. Haque, "Drag force in strongly coupled, anisotropic plasma at finite chemical potential," arXiv:1410.7040 [hep-th]. 3

[34] K. B. Fadafan and H. Soltanpanahi, "Energy loss in a strongly coupled anisotropic plasma," JHEP 1210, 085 (2012) [arXiv:1206.2271 [hep-th]]. 3

[35] B. Muller and D. -L. Yan, "Light Probes in a Strongly Coupled Anisotropic Plasma," Phys. Rev. D 87, 046004 (2013) [arXiv:1210.2095 [hep-th]]. 3

[36] A. Rebhan and D. Steineder, "Probing Two Holographic Models of Strongly Coupled Anisotropic Plasma," JHEP 1208, 020 (2012) [arXiv:1205.4684 [hep-th]]. 3

[37] M. Chernicoff, D. Fernandez, D. Mateos and D. Trancanelli, "Jet quenching in a strongly coupled anisotropic plasma," JHEP 1208, 041 (2012) [arXiv:1203.0561 [hepth]]. $3,70,83,98,100$

[38] M. Chernicoff, D. Fernandez, D. Mateos and D. Trancanelli, "Quarkonium dissociation by anisotropy," JHEP 1301 (2013) 170 [arXiv:1208.2672 [hep-th]]. 3, 71, 83

[39] S. Chakraborty and N. Haque, "Holographic quark-antiquark potential in hot, anisotropic Yang-Mills plasma," Nucl. Phys. B 874, 821 (2013) [arXiv:1212.2769 [hep-th]]. 3

[40] K. B. Fadafan, D. Giataganas and H. Soltanpanahi, "The Imaginary Part of the Static Potential in Strongly Coupled Anisotropic Plasma," JHEP 1311, 107 (2013) [arXiv:1306.2929 [hep-th]]. 3

[41] D. Giataganas and H. Soltanpanahi, "Universal properties of the Langevin diffusion coefficients," Phys. Rev. D 89, 026011 (2014) [arXiv:1310.6725 [hep-th]]. 3

[42] S. Chakrabortty, S. Chakraborty and N. Haque, "Brownian motion in strongly coupled, anisotropic Yang-Mills plasma: A holographic approach," Phys. Rev. D 89, 066013 (2014) [arXiv:1311.5023 [hep-th]]. 3 
[43] D. Giataganas and H. Soltanpanahi, "Heavy Quark Diffusion in Strongly Coupled Anisotropic Plasmas," JHEP 1406, 047 (2014) [arXiv:1312.7474 [hep-th]]. 3

[44] M. Ali-Akbari and H. Ebrahim, "Chiral Symmetry Breaking: To Probe Anisotropy and Magnetic Field in QGP," Phys. Rev. D 89, no. 6, 065029 (2014) [arXiv:1309.4715 [hep-th]]. 3

[45] L. Patino and D. Trancanelli, "Thermal photon production in a strongly coupled anisotropic plasma," JHEP 1302, 154 (2013) [arXiv:1211.2199 [hep-th]]. 3, 75, 76, $77,78,79,83$

[46] S. Y. Wu and D. L. Yang, "Holographic Photon Production with Magnetic Field in Anisotropic Plasmas," JHEP 1308 (2013) 032 [arXiv:1305.5509 [hep-th]]. 3, 75, 77

[47] G. Arciniega, P. Ortega and L. Patino, "Brighter Branes, enhancement of photon production by strong magnetic fields in the gauge/gravity correspondence," JHEP 1404, 192 (2014) [arXiv:1307.1153 [hep-th]]. 3

[48] V. Jahnke, A. Luna, L. Patiño and D. Trancanelli, "More on thermal probes of a strongly coupled anisotropic plasma," JHEP 1401, 149 (2014) [arXiv:1311.5513 [hepth]]. $3,75,76,77,79$

[49] L. Cheng, X. H. Ge and S. J. Sin, "Anisotropic plasma at finite $U(1)$ chemical potential," JHEP 1407, 083 (2014) [arXiv:1404.5027 [hep-th]]. 3

[50] L. Cheng, X. H. Ge and S. J. Sin, "Anisotropic plasma with a chemical potential and scheme-independent instabilities," Phys. Lett. B 734, 116 (2014) [arXiv:1404.1994 [hep-th]]. 3

[51] D. Giataganas, "Observables in Strongly Coupled Anisotropic Theories," PoS Corfu 2012, 122 (2013) [arXiv:1306.1404 [hep-th]]. 3, 70, 83

[52] S. Jain, N. Kundu, K. Sen, A. Sinha and S. P. Trivedi, "A Strongly Coupled Anisotropic Fluid From Dilaton Driven Holography," arXiv:1406.4874 [hep-th]. 3

[53] X. O. Camanho, J. D. Edelstein, J. Maldacena and A. Zhiboedov, "Causality Constraints on Corrections to the Graviton Three-Point Coupling," arXiv:1407.5597 [hepth]. 3

[54] V. Jahnke, A. S. Misobuchi and D. Trancanelli, "The Chern-Simons diffusion rate from higher curvature gravity," Phys. Rev. D 89, 107901 (2014) [arXiv:1403.2681 [hep-th]]. 3, 63

[55] V. Jahnke, A. S. Misobuchi and D. Trancanelli, "Holographic renormalization and anisotropic black branes in higher curvature gravity," JHEP 1501, 122 (2015) [arXiv:1411.5964 [hep-th]]. 3, 82

[56] V. Jahnke and A. S. Misobuchi, "Probing strongly coupled anisotropic plasmas from higher curvature gravity," arXiv:1510.03774 [hep-th]. 3

[57] J. Polchinski, "Dualities of Fields and Strings," arXiv:1412.5704 [hep-th]. 4 
[58] O. Aharony, S. S. Gubser, J. M. Maldacena, H. Ooguri and Y. Oz, "Large N field theories, string theory and gravity," Phys. Rept. 323, 183 (2000) [hep-th/9905111]. 4

[59] A. V. Ramallo, "Introduction to the AdS/CFT correspondence," Springer Proc. Phys. 161, 411 (2015) [arXiv:1310.4319 [hep-th]]. 4

[60] E. D'Hoker and D. Z. Freedman, "Supersymmetric gauge theories and the AdS CFT correspondence," hep-th/0201253. 4

[61] H. Nastase, "Introduction to the AdS/CFT correspondence," Cambridge University Press (2015). 4

[62] M. Ammon and J. Erdmenger, "Gauge/gravity duality : Foundations and applications," 4

[63] G. 't Hooft, "A Planar Diagram Theory for Strong Interactions," Nucl. Phys. B 72, 461 (1974). 5

[64] S. W. Hawking, "Gravitational radiation from colliding black holes," Phys. Rev. Lett. 26, 1344 (1971). 7

[65] J. D. Bekenstein, "Black holes and entropy," Phys. Rev. D 7, 2333 (1973). 7

[66] L. Susskind, "The World as a hologram," J. Math. Phys. 36, 6377 (1995) [hepth/9409089]. 7

[67] M. B. Green and J. H. Schwarz, "Supersymmetrical Dual String Theory," Nucl. Phys. B 181, 502 (1981). doi:10.1016/0550-3213(81)90538-1 13

[68] N. Berkovits, "Super Poincare covariant quantization of the superstring," JHEP 0004, 018 (2000) doi:10.1088/1126-6708/2000/04/018 [hep-th/0001035]. 13

[69] J. Polchinski, "Dirichlet Branes and Ramond-Ramond charges," Phys. Rev. Lett. 75, 4724 (1995) doi:10.1103/PhysRevLett.75.4724 14

[70] E. Witten, "Bound states of strings and p-branes," Nucl. Phys. B 460, 335 (1996) [hep-th/9510135]. 15

[71] T. Eguchi, P. B. Gilkey and A. J. Hanson, "Gravitation, Gauge Theories and Differential Geometry," Phys. Rept. 66, 213 (1980). doi:10.1016/0370-1573(80)90130-1 23

[72] R. C. Myers, "Higher Derivative Gravity, Surface Terms and String Theory," Phys. Rev. D 36, 392 (1987). doi:10.1103/PhysRevD.36.392 23

[73] C. Garraffo and G. Giribet, "The Lovelock Black Holes," Mod. Phys. Lett. A 23, 1801 (2008) [arXiv:0805.3575 [gr-qc]]. 25

[74] D. Kastor, S. Ray and J. Traschen, "Smarr Formula and an Extended First Law for Lovelock Gravity," Class. Quant. Grav. 27, 235014 (2010) [arXiv:1005.5053 [hep-th]]. 26 
[75] D. T. Son and A. O. Starinets, "Minkowski space correlators in AdS / CFT correspondence: Recipe and applications," JHEP 0209 (2002) 042 [hep-th/0205051]. 28, $35,36,78,93,94$

[76] P. Kovtun, D. T. Son and A. O. Starinets, "Viscosity in strongly interacting quantum field theories from black hole physics," Phys. Rev. Lett. 94, 111601 (2005) [hepth/0405231]. 28, 36, 40, 63, 82

[77] H. Liu and A. A. Tseytlin, "D = 4 superYang-Mills, $\mathrm{D}=5$ gauged supergravity, and $\mathrm{D}=4$ conformal supergravity," Nucl. Phys. B 533, 88 (1998) doi:10.1016/S05503213(98)00443-X [hep-th/9804083]. 30

[78] M. Brigante, H. Liu, R. C. Myers, S. Shenker and S. Yaida, "Viscosity Bound Violation in Higher Derivative Gravity," Phys. Rev. D 77, 126006 (2008) [arXiv:0712.0805 [hepth]]. 31

[79] D. M. Hofman, "Higher Derivative Gravity, Causality and Positivity of Energy in a UV complete QFT," Nucl. Phys. B 823, 174 (2009) doi:10.1016/j.nuclphysb.2009.08.001 [arXiv:0907.1625 [hep-th]]. 32

[80] G. T. Horowitz and N. Itzhaki, "Black holes, shock waves, and causality in the AdS / CFT correspondence," JHEP 9902, 010 (1999) doi:10.1088/1126-6708/1999/02/010 [hep-th/9901012]. 32

[81] X. O. Camanho, J. D. Edelstein, J. Maldacena and A. Zhiboedov, "Causality Constraints on Corrections to the Graviton Three-Point Coupling," arXiv:1407.5597 [hepth]. 33

[82] F. R. Klinkhamer and N. S. Manton, "A Saddle Point Solution in the Weinberg-Salam Theory," Phys. Rev. D 30, 2212 (1984). 34

[83] P. B. Arnold and L. D. McLerran, "The Sphaleron Strikes Back," Phys. Rev. D 37, 1020 (1988). 34

[84] D. Kharzeev, R. D. Pisarski and M. H. G. Tytgat, "Possibility of spontaneous parity violation in hot QCD," Phys. Rev. Lett. 81, 512 (1998); [hep-ph/9804221]. 34

[85] G. D. Moore and M. Tassler, "The Sphaleron Rate in SU(N) Gauge Theory," JHEP 1102, 105 (2011). [arXiv:1011.1167 [hep-ph]]. 34

[86] D. E. Kharzeev, L. D. McLerran and H. J. Warringa, "The Effects of topological charge change in heavy ion collisions: 'Event by event P and CP violation'," Nucl. Phys. A 803, 227 (2008); [arXiv:0711.0950 [hep-ph]]. 35

[87] K. Fukushima, D. E. Kharzeev and H. J. Warringa, "The Chiral Magnetic Effect," Phys. Rev. D 78, 074033 (2008). [arXiv:0808.3382 [hep-ph]]. 35

[88] P. B. Arnold, D. Son and L. G. Yaffe, "The Hot baryon violation rate is O (alpha-w**5 T**4)," Phys. Rev. D 55, 6264 (1997) [hep-ph/9609481]. 35 
[89] P. Huet and D. T. Son, "Long range physics in a hot nonAbelian plasma," Phys. Lett. B 393, 94 (1997) [hep-ph/9610259]. 35

[90] D. Bodeker, "On the effective dynamics of soft nonAbelian gauge fields at finite temperature," Phys. Lett. B 426, 351 (1998) [hep-ph/9801430]. 35

[91] G. D. Moore, "Do we understand the sphaleron rate?," hep-ph/0009161. 35

[92] G. Koutsoumbas, E. Papantonopoulos and G. Siopsis, "Shear Viscosity and ChernSimons Diffusion Rate from Hyperbolic Horizons," Phys. Lett. B 677, 74 (2009) [arXiv:0809.3388 [hep-th]]. 35

[93] G. Basar and D. E. Kharzeev, "The Chern-Simons diffusion rate in strongly coupled N=4 SYM plasma in an external magnetic field," Phys. Rev. D 85, 086012 (2012) [arXiv:1202.2161 [hep-th]]. 35

[94] B. Craps, C. Hoyos, P. Surowka and P. Taels, "Chern-Simons diffusion rate in a holographic Yang-Mills theory," JHEP 1211, 109 (2012) [Erratum-ibid. 1302, 087 (2013)] [arXiv:1209.2532 [hep-th]]. 35

[95] U. Gürsoy, I. Iatrakis, E. Kiritsis, F. Nitti and A. O'Bannon, "The ChernSimons Diffusion Rate in Improved Holographic QCD," JHEP 1302, 119 (2013) [arXiv:1212.3894 [hep-th]]. 35, 38

[96] R. C. Myers and B. Robinson, "Black Holes in Quasi-topological Gravity," JHEP 1008, 067 (2010) [arXiv:1003.5357 [gr-qc]]. 35, 36, 37, 40

[97] M. Brigante, H. Liu, R. C. Myers, S. Shenker and S. Yaida, "Viscosity Bound Violation in Higher Derivative Gravity," Phys. Rev. D 77, 126006 (2008) [arXiv:0712.0805 [hepth]]. $35,39,40,43,59,63,65,82$

[98] R. C. Myers, M. F. Paulos and A. Sinha, "Holographic studies of quasi-topological gravity," JHEP 1008, 035 (2010) [arXiv:1004.2055 [hep-th]]. 35, 37, 39, 40

[99] A. Buchel, R. C. Myers and A. Sinha, "Beyond eta/s = 1/4 pi," JHEP 0903, 084 (2009) [arXiv:0812.2521 [hep-th]]. 40, 63

[100] R. -G. Cai, Z. -Y. Nie, N. Ohta and Y. -W. Sun, "Shear Viscosity from Gauss-Bonnet Gravity with a Dilaton Coupling," Phys. Rev. D 79 (2009) 066004 [arXiv:0901.1421 [hep-th]]. 40, 63

[101] J. de Boer, M. Kulaxizi and A. Parnachev, "AdS(7)/CFT(6), Gauss-Bonnet Gravity, and Viscosity Bound," JHEP 1003, 087 (2010) [arXiv:0910.5347 [hep-th]]. 40, 63

[102] X. O. Camanho, J. D. Edelstein and M. F. Paulos, "Lovelock theories, holography and the fate of the viscosity bound," JHEP 1105, 127 (2011) [arXiv:1010.1682 [hepth]]. 40, 63

[103] T. Takahashi and J. Soda, "Pathologies in Lovelock AdS Black Branes and AdS/CFT," Class. Quant. Grav. 29, 035008 (2012) [arXiv:1108.5041 [hep-th]]. 40, 63 
[104] S. Cremonini, "The Shear Viscosity to Entropy Ratio: A Status Report," Mod. Phys. Lett. B 25, 1867 (2011) [arXiv:1108.0677 [hep-th]]. 40, 63

[105] J. Polchinski and E. Silverstein, "Large-density field theory, viscosity, and ' $2 k_{F}$ ' singularities from string duals," Class. Quant. Grav. 29, 194008 (2012) [arXiv:1203.1015 [hep-th]]. 40

[106] J. M. Maldacena and H. Ooguri, "Strings in AdS(3) and SL(2,R) WZW model 1.: The Spectrum," J. Math. Phys. 42, 2929 (2001) [hep-th/0001053]. 40

[107] J. M. Maldacena, H. Ooguri and J. Son, "Strings in $\operatorname{AdS}(3)$ and the $\operatorname{SL}(2, R)$ WZW model. Part 2. Euclidean black hole," J. Math. Phys. 42, 2961 (2001) [hepth/0005183]. 40

[108] J. M. Maldacena and H. Ooguri, "Strings in AdS(3) and the SL(2,R) WZW model. Part 3. Correlation functions," Phys. Rev. D 65, 106006 (2002) [hep-th/0111180]. 40

[109] M. H. Dehghani and R. B. Mann, "Lovelock-Lifshitz Black Holes," JHEP 1007, 019 (2010) [arXiv:1004.4397 [hep-th]]. 45

[110] M. Park and R. B. Mann, "Deformations of Lifshitz holography with the GaussBonnet term in $(n+1)$ dimensions," JHEP 1308, 003 (2013) [arXiv:1305.5578 [hepth]]. 45

[111] K. Skenderis, "Lecture notes on holographic renormalization," Class. Quant. Grav. 19, 5849 (2002) [hep-th/0209067]. 45

[112] L. Y. Hung, R. C. Myers and M. Smolkin, "On Holographic Entanglement Entropy and Higher Curvature Gravity," JHEP 1104, 025 (2011) [arXiv:1101.5813 [hep-th]]. 57

[113] M. M. Caldarelli, R. Emparan and B. Van Pol, "Higher-dimensional Rotating Charged Black Holes," JHEP 1104, 013 (2011) [arXiv:1012.4517 [hep-th]]. 59

[114] K. B. Fadafan, "Charge effect and finite 't Hooft coupling correction on drag force and Jet Quenching Parameter," Eur. Phys. J. C 68 (2010) 505 [arXiv:0809.1336 [hep-th]]. 63, 66, 82

[115] Y. Kats and P. Petrov, "Effect of curvature squared corrections in AdS on the viscosity of the dual gauge theory," JHEP 0901, 044 (2009) [arXiv:0712.0743 [hepth]]. 63

[116] M. Brigante, H. Liu, R. C. Myers, S. Shenker and S. Yaida, "The Viscosity Bound and Causality Violation," Phys. Rev. Lett. 100, 191601 (2008) [arXiv:0802.3318 [hepth]]. 63

[117] X. H. Ge, Y. Matsuo, F. W. Shu, S. J. Sin and T. Tsukioka, "Viscosity Bound, Causality Violation and Instability with Stringy Correction and Charge," JHEP 0810, 009 (2008) [arXiv:0808.2354 [hep-th]]. 63 
[118] X. H. Ge and S. J. Sin, "Shear viscosity, instability and the upper bound of the Gauss-Bonnet coupling constant," JHEP 0905, 051 (2009) [arXiv:0903.2527 [hepth]]. 63

[119] X. H. Ge, S. J. Sin, S. F. Wu and G. H. Yang, "Shear viscosity and instability from third order Lovelock gravity," Phys. Rev. D 80, 104019 (2009) [arXiv:0905.2675 [hep-th]]. 63

[120] N. Iqbal and H. Liu, "Universality of the hydrodynamic limit in AdS/CFT and the membrane paradigm," Phys. Rev. D 79, 025023 (2009) [arXiv:0809.3808 [hep-th]]. 63,64

[121] A. Bhattacharyya and D. Roychowdhury, "Viscosity bound for anisotropic superfluids in higher derivative gravity," JHEP 1503, 063 (2015) [arXiv:1410.3222 [hep-th]]. 63,86

[122] S. S. Gubser, "Drag force in AdS/CFT," Phys. Rev. D 74, 126005 (2006) [hepth/0605182]. 65, 66, 67, 97

[123] C. P. Herzog, A. Karch, P. Kovtun, C. Kozcaz and L. G. Yaffe, "Energy loss of a heavy quark moving through $\mathrm{N}=4$ supersymmetric Yang-Mills plasma," JHEP 0607, 013 (2006) [hep-th/0605158]. 65, 66, 67, 97

[124] J. Casalderrey-Solana and D. Teaney, "Heavy quark diffusion in strongly coupled N=4 Yang-Mills," Phys. Rev. D 74 (2006) 085012 [hep-ph/0605199]. 66

[125] C. P. Herzog, "Energy Loss of Heavy Quarks from Asymptotically AdS Geometries," JHEP 0609 (2006) 032 [hep-th/0605191]. 66

[126] E. Caceres and A. Guijosa, "Drag force in charged N=4 SYM plasma," JHEP 0611 (2006) 077 [hep-th/0605235]. 66

[127] M. Chernicoff and A. Guijosa, "Energy Loss of Gluons, Baryons and k-Quarks in an N=4 SYM Plasma," JHEP 0702 (2007) 084 [hep-th/0611155]. 66

[128] J. F. Vazquez-Poritz, "Drag force at finite 't Hooft coupling from AdS/CFT," arXiv:0803.2890 [hep-th]. 66, 67

[129] L. Cheng, X. H. Ge and S. Y. Wu, "Drag force of Anisotropic plasma at finite $U(1)$ chemical potential," arXiv:1412.8433 [hep-th]. 66

[130] K. B. Fadafan, "R**2 curvature-squared corrections on drag force," JHEP 0812, 051 (2008) [arXiv:0803.2777 [hep-th]]. 66, 67

[131] I. Arsene et al. [BRAHMS Collaboration], "Transverse momentum spectra in $\mathrm{Au}+\mathrm{Au}$ and $\mathrm{d}+\mathrm{Au}$ collisions at $\mathrm{s}^{* *}(1 / 2)=200-\mathrm{GeV}$ and the pseudorapidity dependence of high p(T) suppression," Phys. Rev. Lett. 91, 072305 (2003) 68

[132] S. S. Adler et al. [PHENIX Collaboration], "Absence of suppression in particle production at large transverse momentum in $\mathrm{S}(\mathrm{NN}) * *(1 / 2)=200-\mathrm{GeV} \mathrm{d}+\mathrm{Au}$ collisions," Phys. Rev. Lett. 91, 072303 (2003) [nucl-ex/0306021]. 68 
[133] B. B. Back et al. [PHOBOS Collaboration], "Centrality dependence of charged hadron transverse momentum spectra in $\mathrm{d}+\mathrm{Au}$ collisions at $\mathrm{S}(\mathrm{NN}) * * 1 / 2=200$ GeV," Phys. Rev. Lett. 91, 072302 (2003) [nucl-ex/0306025]. 68

[134] J. Adams et al. [STAR Collaboration], "Evidence from d + Au measurements for final state suppression of high $\mathrm{p}(\mathrm{T})$ hadrons in $\mathrm{Au}+\mathrm{Au}$ collisions at RHIC," Phys. Rev. Lett. 91, 072304 (2003) [nucl-ex/0306024]. 68

[135] A. Majumder and M. Van Leeuwen, "The Theory and Phenomenology of Perturbative QCD Based Jet Quenching," Prog. Part. Nucl. Phys. A 66, 41 (2011) [arXiv:1002.2206 [hep-ph]]. 69

[136] K. M. Burke et al. [JET Collaboration], "Extracting the jet transport coefficient from jet quenching in high-energy heavy-ion collisions," Phys. Rev. C 90, no. 1, 014909 (2014) [arXiv:1312.5003 [nucl-th]]. 69, 70

[137] H. Liu, K. Rajagopal and U. A. Wiedemann, "Calculating the jet quenching parameter from AdS/CFT," Phys. Rev. Lett. 97, 182301 (2006) [hep-ph/0605178]. 69

[138] H. Liu, K. Rajagopal and U. A. Wiedemann, "Wilson loops in heavy ion collisions and their calculation in AdS/CFT," JHEP 0703, 066 (2007) [hep-ph/0612168]. 69

[139] F. D'Eramo, H. Liu and K. Rajagopal, "Transverse Momentum Broadening and the Jet Quenching Parameter, Redux," Phys. Rev. D 84, 065015 (2011) [arXiv:1006.1367 [hep-ph]]. 69

[140] M. Panero, K. Rummukainen and A. Schäfer, "Lattice Study of the Jet Quenching Parameter," Phys. Rev. Lett. 112, no. 16, 162001 (2014) [arXiv:1307.5850 [hep-ph]]. 69

[141] K. Bitaghsir Fadafan, B. Pourhassan and J. Sadeghi, "Calculating the jet-quenching parameter in STU background," Eur. Phys. J. C 71 (2011) 1785 [arXiv:1005.1368 [hep-th]]. 69

[142] J. Sadeghi and B. Pourhassan, "Jet-quenching of the rotating heavy meson in a $\mathcal{N}=4$ SYM plasma in presence of a constant electric field," Int. J. Theor. Phys. 50 (2011) 2305 [arXiv:1001.0706 [hep-th]]. 69

[143] D. Li, J. Liao and M. Huang, "Enhancement of jet quenching around phase transition: result from the dynamical holographic model," Phys. Rev. D 89 (2014) 12, 126006 [arXiv:1401.2035 [hep-ph]]. 69

[144] B. G. Zakharov, "Radiative energy loss of high-energy quarks in finite size nuclear matter and quark - gluon plasma," JETP Lett. 65, 615 (1997) [hep-ph/9704255]. 69

[145] Z. q. Zhang, D. f. Hou and H. c. Ren, "The finite 't Hooft coupling correction on jet quenching parameter in a $\mathcal{N}=4$ Super Yang-Mills Plasma," JHEP 1301, 032 (2013) [arXiv:1210.5187 [hep-th]]. 70 
[146] N. Armesto, J. D. Edelstein and J. Mas, "Jet quenching at finite 't Hooft coupling and chemical potential from AdS/CFT," JHEP 0609, 039 (2006) [hep-ph/0606245]. 70

[147] H. Satz, "The Quark-Gluon Plasma: A Short Introduction," Nucl. Phys. A 862-863 (2011) 4 [arXiv:1101.3937 [hep-ph]]. 71

[148] J. M. Maldacena, "Wilson loops in large N field theories," Phys. Rev. Lett. 80 (1998) 4859 [hep-th/9803002]. 71

[149] S. J. Rey and J. T. Yee, "Macroscopic strings as heavy quarks in large N gauge theory and anti-de Sitter supergravity," Eur. Phys. J. C 22 (2001) 379 [hep-th/9803001]. 71

[150] S. J. Rey, S. Theisen and J. T. Yee, "Wilson-Polyakov loop at finite temperature in large N gauge theory and anti-de Sitter supergravity," Nucl. Phys. B 527 (1998) 171 [hep-th/9803135]. 71, 72

[151] A. Brandhuber, N. Itzhaki, J. Sonnenschein and S. Yankielowicz, "Wilson loops in the large N limit at finite temperature," Phys. Lett. B 434 (1998) 36 [hepth/9803137]. 71

[152] E. Witten, "Anti-de Sitter space, thermal phase transition, and confinement in gauge theories," Adv. Theor. Math. Phys. 2 (1998) 505 [hep-th/9803131]. 71

[153] M. Chernicoff, J. A. Garcia and A. Guijosa, "The Energy of a Moving QuarkAntiquark Pair in an N=4 SYM Plasma," JHEP 0609 (2006) 068 [hep-th/0607089]. 71

[154] J. Noronha and A. Dumitru, "The Heavy Quark Potential as a Function of Shear Viscosity at Strong Coupling," Phys. Rev. D 80 (2009) 014007 [arXiv:0903.2804 [hepph]]. 71

[155] S. I. Finazzo and J. Noronha, "Estimates for the Thermal Width of Heavy Quarkonia in Strongly Coupled Plasmas from Holography," JHEP 1311 (2013) 042 [arXiv:1306.2613 [hep-ph]]. 71

[156] S. I. Finazzo and J. Noronha, "Thermal suppression of moving heavy quark pairs in a strongly coupled plasma," JHEP 1501 (2015) 051 [arXiv:1406.2683 [hep-th]]. 71

[157] S. I. Finazzo and J. Noronha, "Debye screening mass near deconfinement from holography," Phys. Rev. D 90 (2014) 11, 115028 [arXiv:1411.4330 [hep-th]]. 71

[158] R. Rougemont, R. Critelli and J. Noronha, "Anisotropic heavy quark potential in strongly-coupled $\mathcal{N}=4$ SYM in a magnetic field," Phys. Rev. D 91 (2015) 6, 066001 [arXiv:1409.0556 [hep-th]]. 71, 73

[159] K. B. Fadafan, "Heavy quarks in the presence of higher derivative corrections from AdS/CFT," Eur. Phys. J. C 71, 1799 (2011) [arXiv:1102.2289 [hep-th]]. 71, 73, 82

[160] A. Dumitru, Y. Guo and M. Strickland, "The Heavy-quark potential in an anisotropic (viscous) plasma," Phys. Lett. B 662 (2008) 37 [arXiv:0711.4722 [hepph]]. 73 
[161] S. Caron-Huot, P. Kovtun, G. D. Moore, A. Starinets and L. G. Yaffe, "Photon and dilepton production in supersymmetric Yang-Mills plasma," JHEP 0612, 015 (2006) [hep-th/0607237]. 75, 77, 81

[162] D. Mateos and L. Patiño, "Bright branes for strongly coupled plasmas," JHEP 0711, 025 (2007) [arXiv:0709.2168 [hep-th]]. 75, 77

[163] A. Nata Atmaja and K. Schalm, "Photon and Dilepton Production in Soft Wall AdS/QCD," JHEP 1008, 124 (2010) [arXiv:0802.1460 [hep-th]]. 75

[164] Y. Y. Bu, "Photoproduction and conductivity in dense holographic QCD," Phys. Rev. D 86, 026003 (2012); 75

[165] B. Hassanain and M. Schvellinger, "Plasma conductivity at finite coupling," JHEP 1201, 114 (2012) [arXiv:1108.6306 [hep-th]]. 75

[166] B. Hassanain and M. Schvellinger, "Diagnostics of plasma photoemission at strong coupling," Phys. Rev. D 85, 086007 (2012) [arXiv:1110.0526 [hep-th]]. 75

[167] R. Baier, S. A. Stricker, O. Taanila and A. Vuorinen, "Production of Prompt Photons: Holographic Duality and Thermalization," Phys. Rev. D 86, 081901 (2012) [arXiv:1207.1116 [hep-ph]]. 75

[168] D. Steineder, S. A. Stricker and A. Vuorinen, "Holographic Thermalization at Intermediate Coupling," Phys. Rev. Lett. 110, no. 10, 101601 (2013) [arXiv:1209.0291 [hep-ph]]. 75

[169] B. Hassanain and M. Schvellinger, "Plasma photoemission from string theory," JHEP 1212, 095 (2012) [arXiv:1209.0427 [hep-th]]. 75, 81, 82

[170] B. Muller, S. Y. Wu and D. L. Yang, "Elliptic flow from thermal photons with magnetic field in holography," Phys. Rev. D 89 (2014) 2, 026013 [arXiv:1308.6568 [hep-th]]. 75

[171] Y. Bu, "Gauss-Bonnet correction to the $R$-current correlator in $\mathcal{N}=4$ theory at strong coupling," Phys. Rev. D 89, no. 8, 086008 (2014). 75, 79, 82

[172] S. I. Finazzo and R. Rougemont, "Thermal photon and dilepton production and electric charge transport in a baryon rich strongly coupled QGP from holography," arXiv:1510.03321 [hep-ph]. 75

[173] M. Le Bellac, "Thermal Field Theory," Cambridge University Press (1996). 75

[174] M. Ali-Akbari and K. Bitaghsir Fadafan, "Rotating mesons in the presence of higher derivative corrections from gauge-string duality," Nucl. Phys. B 835, 221 (2010) [arXiv:0908.3921 [hep-th]]. 82

[175] M. Ali-Akbari and K. B. Fadafan, "Conductivity at finite 't Hooft coupling from AdS/CFT," Nucl. Phys. B 844, 397 (2011) [arXiv:1008.2430 [hep-th]]. 82

[176] K. B. Fadafan and S. K. Tabatabaei, "The Imaginary Potential and Thermal Width of Moving Quarkonium from Holography," arXiv:1501.00439 [hep-th]. 82 
[177] K. B. Fadafan and S. K. Tabatabaei, "Thermal Width of Quarkonium from Holography," Eur. Phys. J. C 74, 2842 (2014) [arXiv:1308.3971 [hep-th]]. 82

[178] G. Policastro, D. T. Son and A. O. Starinets, "From AdS/CFT correspondence to hydrodynamics," JHEP 0209, 043 (2002) [arXiv:hep-th/0205052]. 93

[179] G. Policastro, D. T. Son and A. O. Starinets, "From AdS/CFT correspondence to hydrodynamics. 2. Sound waves," JHEP 0212, 054 (2002) [hep-th/0210220]. 93

[180] P. K. Kovtun and A. O. Starinets, "Quasinormal modes and holography," Phys. Rev. D 72, 086009 (2005) [hep-th/0506184]. 93

[181] Y. Kinar, E. Schreiber and J. Sonnenschein, "Q anti-Q potential from strings in curved space-time: Classical results," Nucl. Phys. B 566 (2000) 103 [hep-th/9811192]. 102 